\title{
How to build a water-splitting machine: structural insights into photosystem II assembly
}

Jure Zabret

Ruhr University Bochum

\section{Stefan Bohn}

Max Planck Institute of Biochemistry

\section{Sandra Schuller}

Max Planck Institute of Biochemistry

\section{Oliver Arnolds}

Ruhr University Bochum https://orcid.org/0000-0003-1433-1233

\section{Madeline Möller}

Ruhr University Bochum

Jakob Meier-Credo

Max Planck Institute of Biophysics

\section{Pasqual Liauw}

Ruhr University Bochum

\section{Aaron Chan}

University of Illinois at Urbana-Champaign

\section{Emad Tajkhorshid}

University of Illinois at Urbana-Champaign

Julian Langer

Max Planck Institute of Biophysics

\section{Raphael Stoll}

Ruhr University Bochum

\section{Anja Krieger-Liszkay}

Université Paris-Saclay

\section{Benjamin Engel}

Helmholtz Zentrum München

\section{Till Rudack}

Ruhr University Bochum

Jan Schuller

Phillips University Marburg https://orcid.org/0000-0002-9121-1764

Marc Nowaczyk ( $\square$ Marc.M.Nowaczyk@rub.de)

Ruhr University Bochum https://orcid.org/0000-0002-9269-0672 


\section{Article}

Keywords: photosynthesis, photosystem II biogenesis, assembly factors, bicarbonate binding, reactive 15 oxygen species, protection mechanisms, oxygen evolving complex, photoactivation, cryo-EM

Posted Date: October 23rd, 2020

DOI: https://doi.org/10.21203/rs.3.rs-88039/v1

License: (c) (i) This work is licensed under a Creative Commons Attribution 4.0 International License.

Read Full License

Version of Record: A version of this preprint was published at Nature Plants on April 12th, 2021. See the published version at https://doi.org/10.1038/s41477-021-00895-0. 


\title{
How to build a water-splitting machine: structural insights into photosystem II assembly
}

Jure Zabret ${ }^{1 \#}$, Stefan Bohn" ${ }^{2 \#}$, Sandra K. Schuller ${ }^{3,4}$, Oliver Arnolds ${ }^{5}$, Madeline Möller ${ }^{1}$, Jakob Meier-Credo ${ }^{6}$, Pasqual Liauw ${ }^{1}$, Aaron Chan $^{7}$, Emad Tajkhorshid ${ }^{7}$, Julian D. Langer ${ }^{6,8}$, Raphael Stoll ${ }^{5}$, Anja Krieger-Liszkay ${ }^{9}$, Benjamin D. Engel ${ }^{2,10,11}$, Till Rudack ${ }^{12,13, *}$, Jan M. Schuller ${ }^{3,4, *}$, Marc M. Nowaczyk ${ }^{1, *}$

\author{
Affiliations: \\ ${ }^{1}$ Department of Plant Biochemistry, Faculty of Biology \& Biotechnology, Ruhr University \\ Bochum, 44780 Bochum, Germany. \\ ${ }^{2}$ Department of Molecular Structural Biology, Max Planck Institute of Biochemistry, 82152 \\ Martinsried, Germany. \\ ${ }^{3}$ Department of Structural Cell Biology, Max Planck Institute of Biochemistry, 82152 \\ Martinsried, Germany. \\ ${ }^{4}$ SYNMIKRO Research Center, Philipps-University Marburg, 35043 Marburg, Germany. \\ ${ }^{5}$ Biomolecular Spectroscopy and RUBiospek|NMR, Faculty of Chemistry and Biochemistry, \\ Ruhr-University Bochum, 44780 Bochum, Germany \\ ${ }^{6}$ Department of Molecular Membrane Biology, Max Planck Institute of Biophysics, 60438 \\ Frankfurt/Main, Germany.
}

${ }^{7} \mathrm{NIH}$ Center for Macromolecular Modeling and Bioinformatics, Beckman Institute for Advanced Science and Technology, Department of Biochemistry, and Center for Biophysics and Quantitative Biology, University of Illinois at Urbana-Champaign, Urbana, Illinois

${ }^{8}$ Max Planck Institute for Brain Research, Max von Laue Strasse 4, 60438 Frankfurt/Main, Germany.

${ }^{9}$ Université Paris-Saclay, CEA, CNRS, Institute for Integrative Biology of the Cell (I2BC), 91198, Gif-sur-Yvette, France

${ }^{10}$ Helmholtz Pioneer Campus, Helmholtz Zentrum München, Ingolstädter Landstraße 1, 85764 Neuherberg, Germany.

${ }^{11}$ Department of Chemistry, Technical University of Munich, Lichtenbergstraße 4, 85748 Garching, Germany.

${ }^{12}$ Biospectroscopy, Center for Protein Diagnostics (ProDi), Ruhr University Bochum, 44801 Bochum, Germany.

${ }^{13}$ Department of Biophysics, Faculty of Biology \& Biotechnology, Ruhr University Bochum, 44780 Bochum, Germany.

\#Equal contribution

*corresponding authors: till.rudack@,rub.de, jan.schuller@synmikro.uni-marburg.de, marc.m.nowaczyk@,rub.de

\section{One Sentence Highlight:}

The high-resolution Cryo-EM structure of the photosystem II assembly intermediate PSII-I reveals how nature's water splitting catalyst is assembled, protected and prepared for photoactivation by help of the three assembly factors Psb27, Psb28 and Psb34. 


\section{Abstract}

2 Biogenesis of photosystem II (PSII), nature's water splitting catalyst, is assisted by auxiliary

3 proteins that form transient complexes with PSII components to facilitate stepwise assembly events. Using cryo-electron microscopy, we solved the structure of such a PSII assembly intermediate with $2.94 \AA$ resolution. It contains three assembly factors (Psb27, Psb28, Psb34) and provides detailed insights into their molecular function. Binding of Psb28 induces large conformational changes at the PSII acceptor side, which distort the binding pocket of the mobile quinone $\left(\mathrm{Q}_{\mathrm{B}}\right)$ and replace bicarbonate with glutamate as a ligand of the non-heme iron, a structural motif found in reaction centers of non-oxygenic photosynthetic bacteria. These results reveal novel mechanisms that protect PSII from damage during biogenesis until water splitting is activated. Our structure further demonstrates how the PSII active site is prepared for the incorporation of the $\mathrm{Mn}_{4} \mathrm{CaO}_{5}$ cluster, which performs the unique water splitting reaction.

\section{Keywords}

photosynthesis, photosystem II biogenesis, assembly factors, bicarbonate binding, reactive oxygen species, protection mechanisms, oxygen evolving complex, photoactivation, cryo-EM

\section{Introduction}

Photosystem II (PSII) is the only enzyme that catalyzes the light-driven oxidation of water, a thermodynamically demanding reaction that drives photosynthesis, sustaining life on our planet $^{1-3}$. This multi-subunit membrane protein complex is located in the thylakoid membranes of cyanobacteria, algae and plants. PSII strips electrons from water and injects them into the photosynthetic electron transport chain (PET). It forms a homodimer with a molecular mass of $\sim 500 \mathrm{kDa}^{4}$, with each monomer composed of at least 20 protein subunits and numerous cofactors, including chlorophylls, quinones, carotenoids, lipids, bicarbonate and the unique $\mathrm{Mn}_{4} \mathrm{CaO}_{5}$ cluster $^{5-7}$. The two core proteins D1 and D2 form a central, membrane-intrinsic heterodimer, which binds all important redox cofactors involved in internal electron transfer ${ }^{8}$. Light-excitation leads to a charge-separated state in which an electron is transferred from the chlorophyll assembly $\mathrm{P}_{680}{ }^{9}$ to the nearby pheophytin ${ }^{10}$. Subsequently, the electron is passed to the bound plastoquinone $\left(\mathrm{Q}_{\mathrm{A}}\right)$ and then to the mobile plastoquinone molecule $\left(\mathrm{Q}_{\mathrm{B}}\right)$, which leaves the complex after accepting two electrons and two protons ${ }^{11}$. The electron hole at $\mathrm{P}_{680}$ is filled by oxidation of an adjacent tyrosine residue $\left(\mathrm{Tyr}_{\mathrm{z}}\right)^{12}$ and finally by the oxygen evolving 
complex (OEC) that contains the $\mathrm{Mn}_{4} \mathrm{CaO}_{5}$ cluster. In cyanobacteria, the cluster is shielded at the luminal side by the three extrinsic proteins, PsbO, PsbU and PsbV, which regulate access to the OEC by forming a complex network of channels for different substrates and products ${ }^{13}$. Light energy is collected and funneled towards $\mathrm{P}_{680}$ by the two membrane-intrinsic antenna proteins CP43 and CP47. These proteins bind most of the chlorophyll molecules and are located at opposite sides of the D1/D2 heterodimer ${ }^{14}$. Moreover, at least twelve small transmembrane subunits with one or two transmembrane helices have been identified in PSII ${ }^{15}$, including cytochrome- $b_{559}{ }^{16}$.

Structural and spectroscopic investigations have revealed these aforementioned comprehensive insights into PSII function ${ }^{17-21}$, but we are far from understanding PSII biogenesis with molecular detail. How nature facilitates the assembly of a multi-subunit, multi-cofactor membrane protein complex is a fundamental unsolved question. The biogenesis of PSII is even more challenging, as the mature complex performs sophisticated and extreme redox chemistry to catalyze the light-driven oxidation of water. This can easily lead to the formation of reactive oxygen species (e.g., singlet oxygen is produced by triplet chlorophyll in the PSII reaction center) and subsequent loss of function due to damaged proteins and cofactors ${ }^{22,23}$. Biogenesis intermediates with only partially functional fragments of the redox chain are particularly prone to damage, thus demanding specialized protection mechanisms for the assembly process. Therefore, PSII biogenesis is not a spontaneous process but rather must be tightly regulated by the action of assembly factors. Thus far, more than 20 auxiliary proteins have been identified that guide the stepwise assembly of PSII subunits and cofactors via intermediate modules, which are assembled independently and then joined together to produce mature PSII ${ }^{24-26}$. In cyanobacteria, PSII biogenesis begins with the formation of the D1/D2 heterodimer reaction center (RC) complex from the D1 precursor protein (pD1) and the D2 protein. This is assisted by the PSII assembly factor Ycf48 after partial processing of the D1 C-terminal extension by the D1 specific peptidase $\mathrm{CtpA}^{27,28}$. In the next step, the assembly factor Psb28 helps CP47 join the RC complex to form the RC47 complex, in which iD1 is further processed to its mature form by $\mathrm{CtpA}^{29,30}$. Almost all ligands of the $\mathrm{Mn}_{4} \mathrm{CaO}_{5}$ cluster are already present at this stage, except for those provided by CP43, which comes pre-constructed with assembly factor Psb27 and several small subunits (together called the CP43 module) ${ }^{31}$. Psb28 is released as CP43 binds, and the resulting Psb27-PSII monomer is activated by maturation of the OEC and the binding of the extrinsic proteins PsbO, PsbU and $\mathrm{PsbV}^{32-34}$. Finally, PSII biogenesis completes with dimerization of two fully assembled monomers and attachment of the soluble phycobilisome antenna complexes. Interestingly, deletion of $p s b J$, which encodes a small single 
transmembrane helix protein at the entrance of the PSII plastoquinone channel, leads to massive accumulation of an intermediate monomeric PSII complex, which contains both assembly factors Psb27 and Psb28 35 .

Physiological studies of Psb27 and Psb28 deletion strains point towards multifaceted functions. Cyanobacterial mutants lacking Psb28 exhibited slower autotrophic growth, particularly under stress conditions ${ }^{30,36}$, and limited synthesis of Chl-binding proteins but without decrease in PSII functionality ${ }^{30}$. The Psb28 mutant also exhibited an overall increase in PSII repair and faster recovery from photodamage ${ }^{30}$. Chemical cross-linking combined with mass spectrometry revealed that Psb28 binds to the cytosolic side of CP47 close to cytochrome- $\mathrm{b}_{559}$ and the Q blocks electron transport to the acceptor side of PSII, thereby protecting the RC47 complex from excess photodamage during the assembly process ${ }^{37}$. This hypothesis is strengthened by the observation that Psb28 is also found in PSII repair complexes ${ }^{38}$. The luminal PSII assembly factor Psb27 has been similarly well investigated. This lipoprotein is predominantly associated with inactive PSII fractions involved in assembly or repair ${ }^{31,33,38-42}$ stabilizing the CP43 luminal domain and presumably facilitating the assembly of the OEC.

Our current knowledge of PSII biogenesis mainly describes the order of events and protein composition of each intermediate, as well as the general roles of PSII assembly factors. However, the precise molecular functions of these intermediate complexes and the involved assembly factors are still elusive due to their low abundance and intrinsic instability. Highresolution structural information is of vital importance to gain a deeper understanding into the molecular action of PSII assembly factors, as they are proposed to alter the structures of their associated PSII proteins to provide protection or facilitate specific biogenesis transitions.

Here, we use cryo-EM single particle analysis to describe the first molecular structure of a PSII assembly intermediate. This structure represents one of the key transitions in PSII biogenesis: the attachment of the CP43 module to the pre-assembled RC47 reaction center complex, which precedes incorporation and activation of the $\mathrm{Mn}_{4} \mathrm{CaO}_{5}$ cluster. We complement this structural data with spectroscopic analysis, revealing the first detailed insights into the molecular mechanisms of PSII assembly. Our study provides mechanistic answers to three long-standing questions: i) How do assembly factors modulate the structures of PSII subunits to assist biogenesis? ii) How is PSII protected from photodamage during assembly? iii) How is the PSII active site prepared for incorporation of the $\mathrm{Mn}_{4} \mathrm{CaO}_{5}$ cluster? 


\section{Results}

\section{Structure determination of the PSII assembly intermediate (PSI-I)}

Stable PSII intermediates were purified from the T. elongatus $\Delta p s b J$ mutant ${ }^{35}$ by affinity chromatography using a twin-strep-tag fused to the C-terminus of the CP43 subunit and subsequent ion exchange chromatography (Fig. S1A). The main peak of the IEC profile corresponds primarily to monomeric PSII, which lacks the extrinsic subunits PsbO, PsbU and PsbV that are indicative for water splitting activity (Fig. S1B and C). Single particle cryo-EM analysis of this PSII fraction resulted in three different high-resolution maps that allowed model building with high confidence and excellent statistics (Fig. S2, Table S1). In addition to the protein subunits, we also faithfully assigned all essential non-protein cofactors, including chlorophylls, quinones, carotenoids and lipids, which are also present in the mature PSII complex (Fig. S3). Consistent with previous biochemical studies ${ }^{33,35,40}$, the EM density corresponding to the fully assembled, active $\mathrm{Mn}_{4} \mathrm{CaO}_{5}$ cluster is missing in the purified biogenesis intermediates. The first cryo-EM map (2.94 $\AA$ ), which we call PSII-I (for PSIIIntermediate), provides a snapshot of the attachment of the CP43 module to the pre-assembled RC47 reaction center complex (Fig. 1). This PSII intermediate contains three assembly factors (Psb27, Psb28 and Psb34), as well as almost all the membrane-intrinsic subunits and cofactors found in mature PSII. Psb27 and Psb28 are well-known assembly factors ${ }^{30,31,34,35,43}$, whereas the additional single transmembrane helix protein (ts10063), which we named Psb34, has not been described before. The small subunit PsbY, which is known to be loosely bound ${ }^{44}$, is not resolved in our structure. In addition, PsbJ is not present, as the corresponding gene was inactivated to stall PSII assembly at this specific transition ${ }^{35}$.

The two additional maps serve as internal controls. PSII-I' (2.76 ̊) lacks Psb27 but is otherwise comparable to PSII-I; the root mean square deviation (RMSD) of the $\mathrm{C}_{\alpha}$ atomic positions between similar subunits of the two complexes is $0.4 \AA$. Most likely, Psb27 was partly lost during sample preparation. The third cryo-EM map (2.82 $\AA$ ), which we call PSII-M (for PSIIMonomer), represents a monomeric PSII complex without bound assembly factors. Comparison of our PSII-M structure with a crystal structure of monomeric PSII ${ }^{44}$ (PDB-ID 3KZI, $3.6 \AA$ ) reveals only minimal differences between both structures, with a C $\alpha$ RMSD of $1.3 \AA$, which verifies that the structural changes observed in PSII-I are not caused by the deletion of PsbJ. 


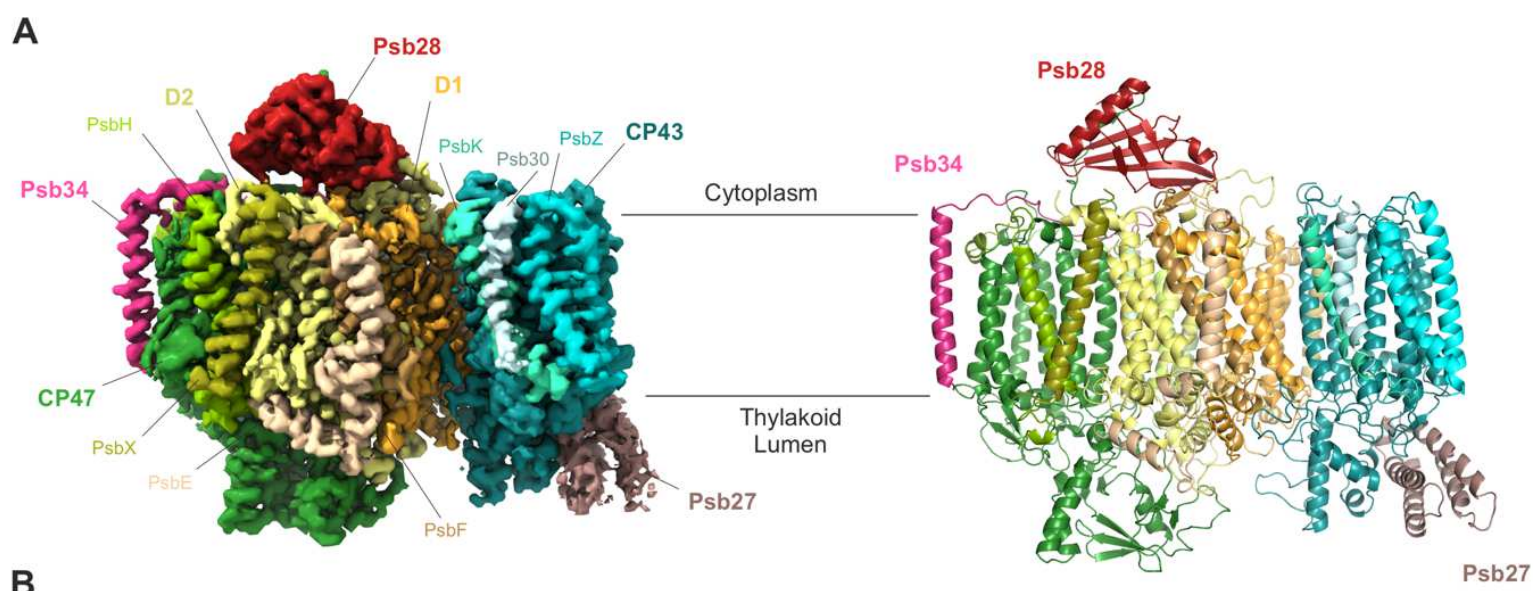

B
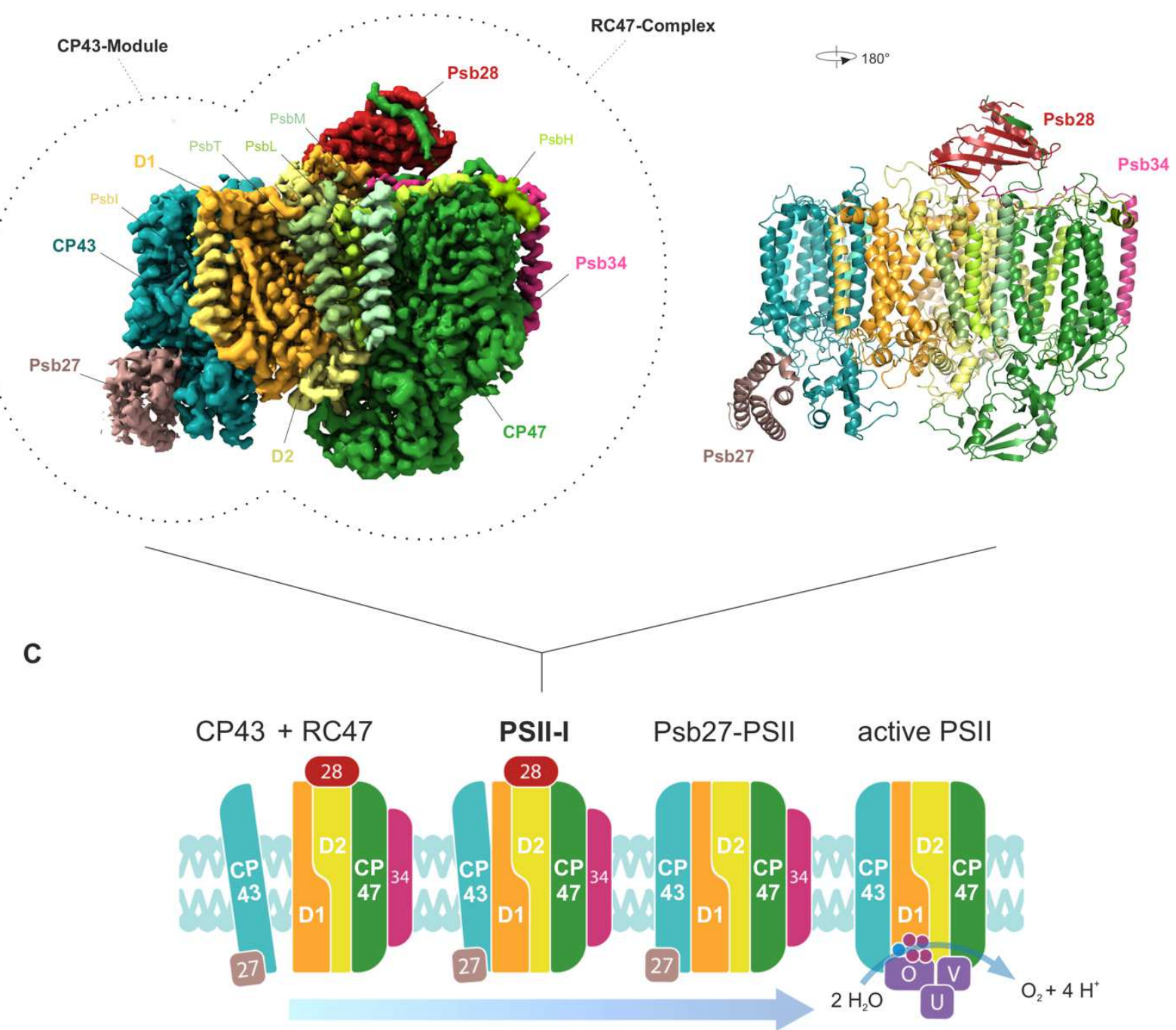

Fig. 1: Cryo-EM map of a PSII assembly intermediate (PSII-I) from T. elongatus, segmented by subunit. (A) 15 PSII subunits and 3 assembly factors are colored and named (PSII subunits: D1, D2, CP43, CP47, PsbE, PsbF, PsbH, PsbI, PsbK, PsbL, PsbM, PsbT, PsbX, PsbZ and Psb30; assembly factors: Psb27, Psb28 and ts10063, which we named Psb34) (front view). (B) Parts of PSII that originate from the CP43 module (comprised of CP43, Psb27, PsbZ, Psb30 and PsbK) and the RC47 complex are indicated by dashed lines (back view). Schematic model of the PSII assembly process starting with the formation of PSII-I from the CP43 module and RC47. Small PSII subunits were omitted for simplicity. 
129 Our PSII-I structure provides the first identification of the single transmembrane helix protein 130 Psb34 bound to a PSII assembly intermediate (Fig. 2A), which we also confirmed by mass 131 spectrometry (Fig. 2B). Psb34 was probably overlooked previously due to its hydrophobicity 132 and small size. It has a single transmembrane helix that binds to the CP47 antenna protein in 133 close proximity to PsbH (Fig. 2A). Its conserved long N-terminal arm is located at the side and 134 top of the D2 subunit (Fig. 2A). In addition, we independently confirmed the interaction of 135 Psb34 with PSII assembly intermediates by isolation of strep-tagged Psb34 complexes, 136 indicating a specific function of Psb34 in the attachment of CP43 to RC47 (Fig. 2C). Two 137 distinct PSII intermediates were isolated via pulldown of strep-tagged Psb34: the RC47 138 complex with bound Psb28 and the subsequent PSII intermediate after attachment of CP43 and 139 Psb27 (Fig. 2C). This observation implies that Psb28 is usually released from the PSII 140 intermediate after attachment of CP43, probably after incorporation of PsbJ, as this trigger is 141 missing in the analyzed $\Delta p s b J$ mutant. Psb34 shows sequence similarity to high-light inducible 142 proteins (HLIPs), which play a role in transient chlorophyll storage and chlorophyll 143 biosynthesis ${ }^{45}$. However, the chlorophyll binding motive is missing in Psb34 (Table S2), 144 suggesting a distinct function for this protein in PSII biogenesis. 

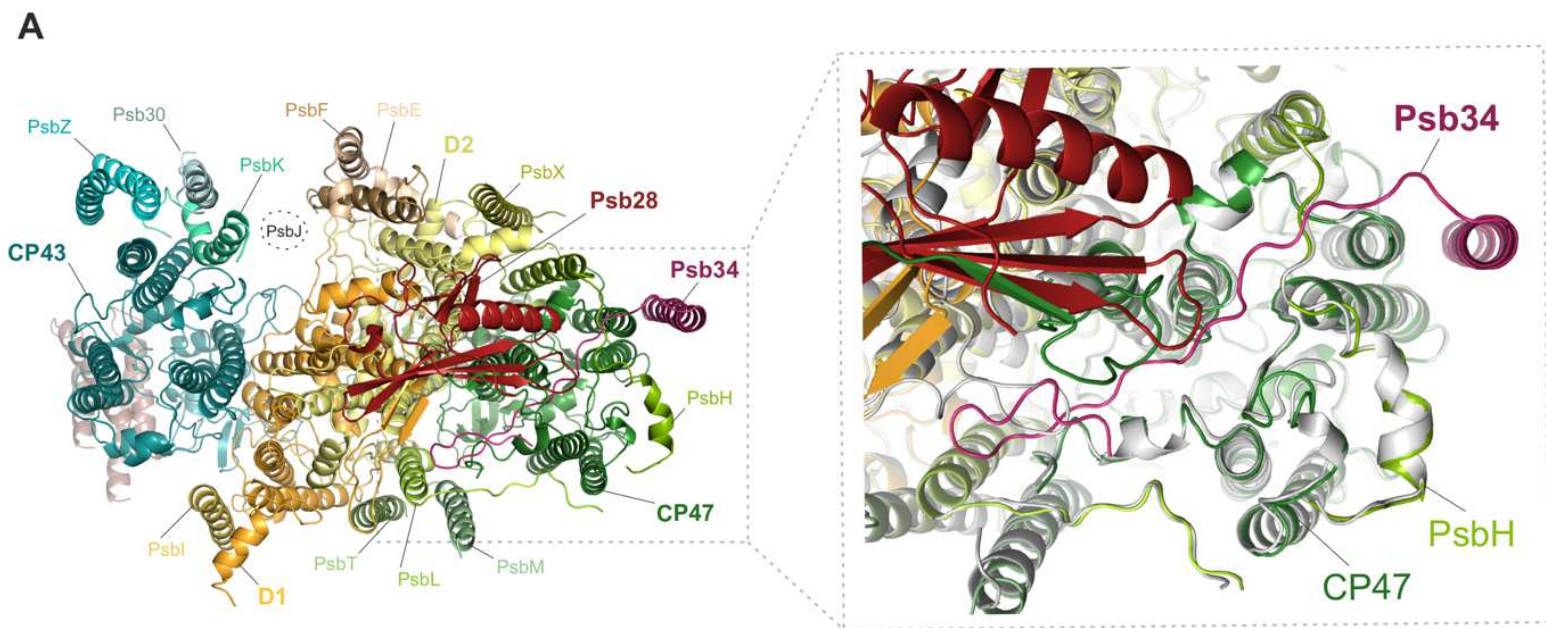

B
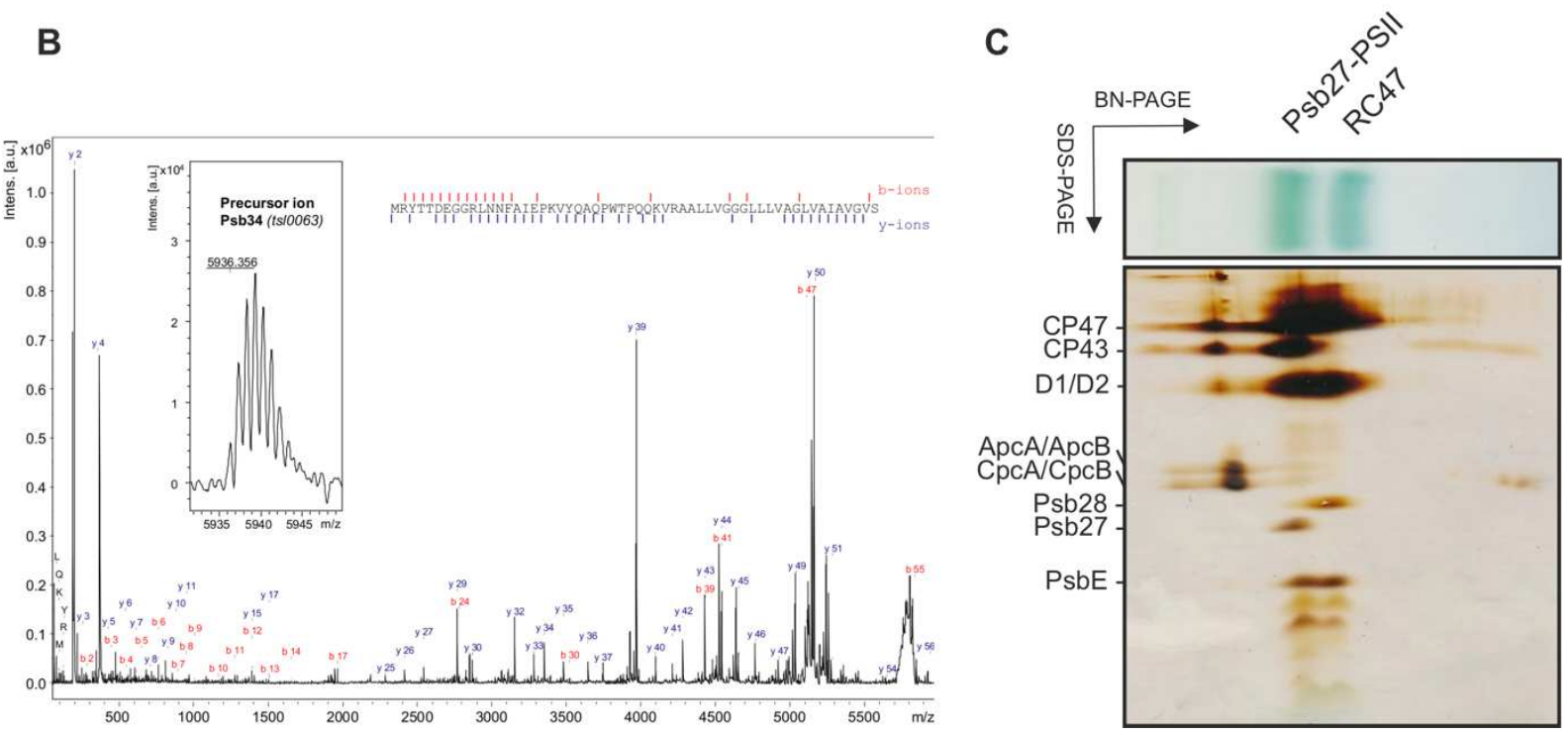

Fig. 2: Psb34 binds to RC47 during attachment of the CP43 module. (A) Binding site of Psb34 at CP47, close to PsbH (top view), with extended binding of the Psb34 N-terminus along the cytoplasmic PSII surface (dashed box). (B) MALDI-ToF analysis of PSII assembly intermediates. Mass spectrum of Psb34 (ts10063) from the PSII complex (inset) and the fragment spectrum obtained for $\mathrm{m} / \mathrm{z} 5936.356$ with annotated $\mathrm{b}$ - and $\mathrm{y}$-ion series matching the Psb34 sequence. Observed fragmentation sites are indicated by dashes in the sequence. Mascot score: 171. (C) Subunit composition of Psb34-PSII assembly intermediates analyzed by 2D-PAGE.

Psb28 forms an extended beta hairpin structure that involves the D1 D-E loop and the

\section{CP47 C-terminus}

147 Psb28 binds on the cytosolic faces of the D1 and D2 subunits, directly above the Qв binding

148 site (Fig. 3A), which differs from the position that was previously predicted by mass 149 spectrometry ${ }^{37}$. Its binding induces the formation of an extended beta-hairpin structure that 150 incorporates the central anti-parallel beta-sheet of Psb28, the C-terminus of CP47 and the D1 151 D-E loop ${ }^{46}$ (Fig. 3A). Binding of Psb28 to the C-terminus of CP47 also imparts a directionality 152 to the assembly process. In the Psb28-free complex (PSII-M), the CP47 C-terminus blocks the 
153 Psb28 binding site by interacting with the D1 D-E loop, thus preventing the reverse process and 154 perturbation of active PSII by Psb28. Using nuclear magnetic resonance (NMR) spectroscopy, 155 we performed chemical shift perturbation (CSP) experiments with recombinant Psb28 and a 156 synthetic peptide of the conserved CP47 C-terminus to characterize this interaction in detail 157 and determine the dissociation constant $\left(K_{D}\right)$ (Fig. 3 and Fig. S4). The CSP measurements 158 indicated significant shifts with a chemical shift difference $(\Delta \delta)$ of more than one standard 159 deviation located at strands $\beta 3$ and $\beta 4$ as well at the C-terminal region of Psb28 (Fig. 3C and 160 D). Upon peptide binding, resonances for several residues gradually appeared with increasing 161 peptide concentration, which were line-broadened beyond detection for the free form of Psb28. 162 This observation indicates a less dynamic and more rigid complex structure. This is further 163 supported by the heteronuclear Overhauser effect (NOE) data, which show that the C-terminus 164 of Psb28 becomes rigid from L108 to K112 upon CP47 peptide binding due to creation of an 165 intermolecular $\beta$-sheet (Fig. 3E). 2D-lineshape analysis was performed, yielding a $\mathrm{K}_{\mathrm{D}}$ of $16653.92 \pm 0.41 \mu \mathrm{M}$ and a dissociation rate $\mathrm{k}_{\text {off }}$ of $10.14 \pm 0.16 \mathrm{~s}^{-1}$, which is consistent with the 167 observed slow-exchange in the NMR spectra (Fig. 3B). The affinity of Psb28 for full-length $168 \mathrm{CP} 47$ and PSII might indeed be even higher due to additional contacts between Psb28 and the 169 D-E loop of D1 (Fig 3A). 


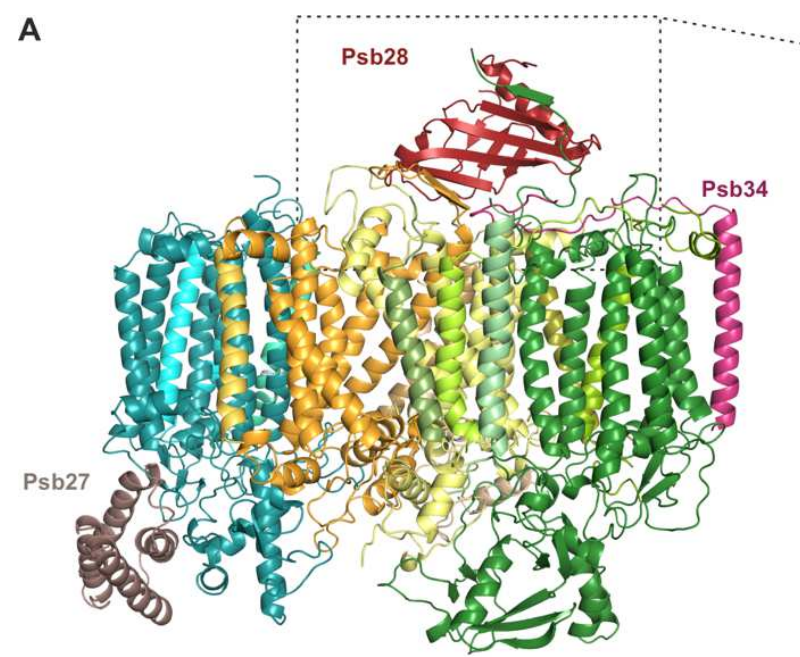

B

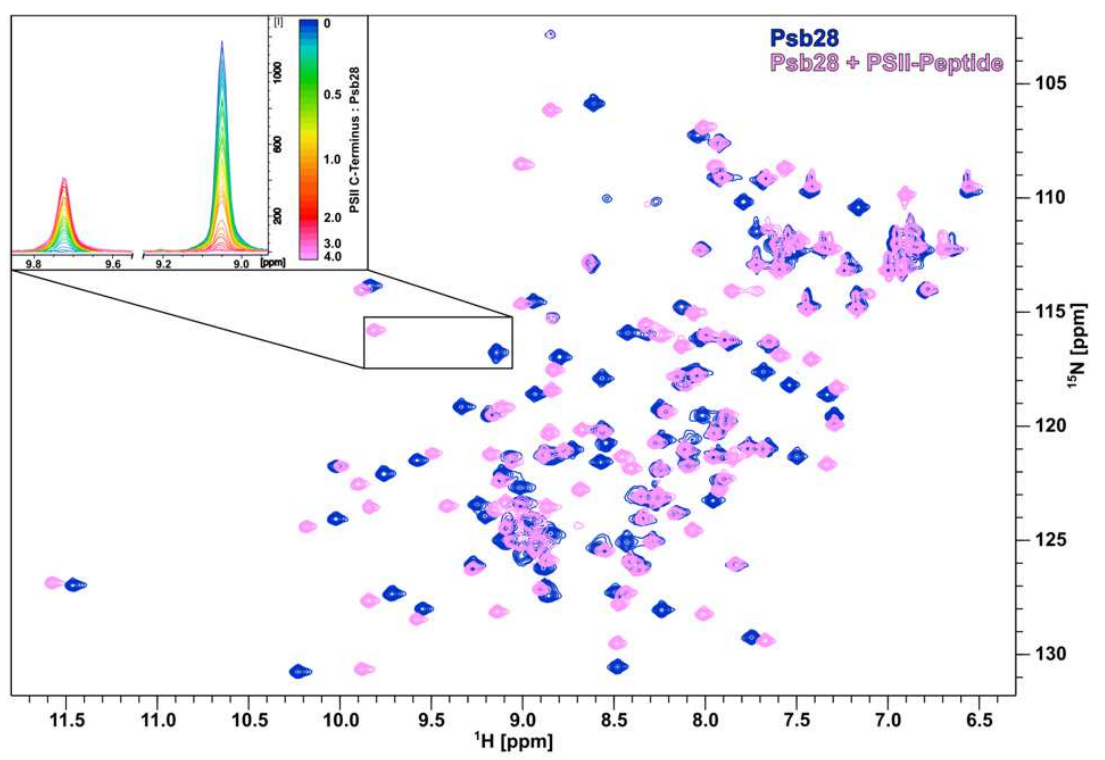

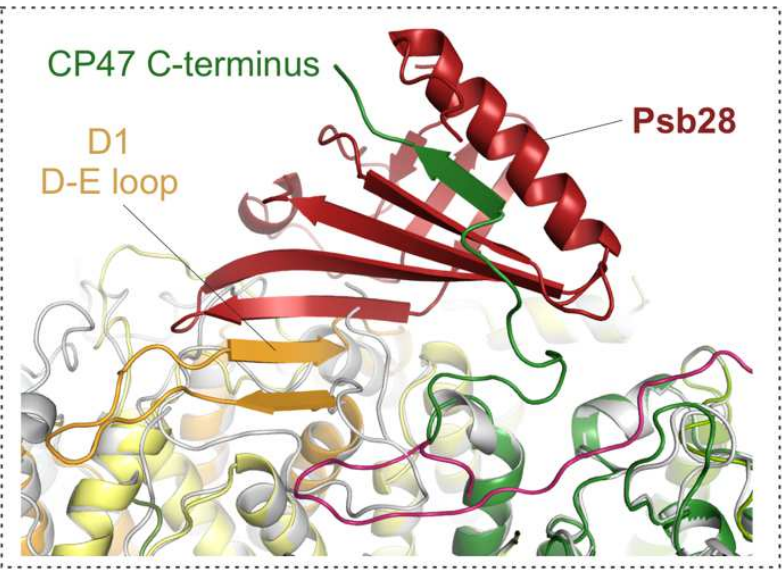

C

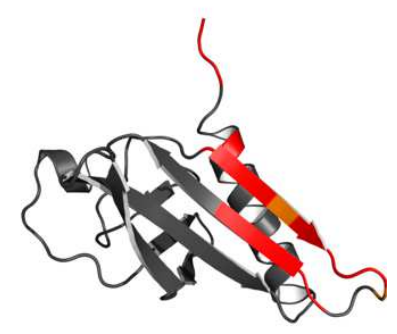

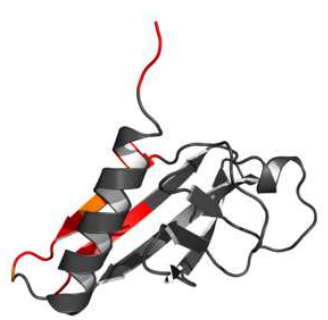

D

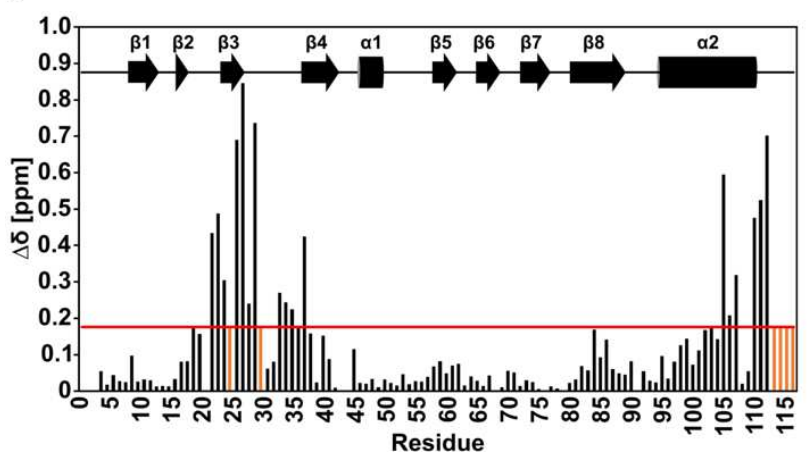

E

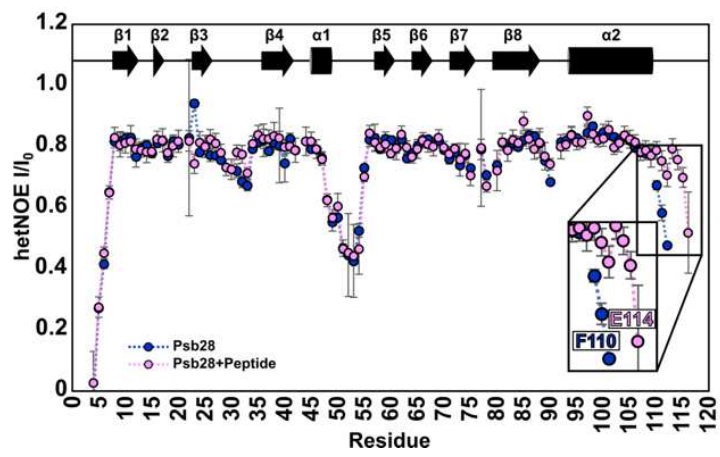

Fig. 3: The role of the CP47 C-terminus in binding of Psb28. (A). Binding of Psb28 at the cytoplasmic/stromal PSII surface (side view, colors correspond to Fig. 1) and continuation of the central Psb28 beta-sheet by the CP47 C-terminus and the D-E loop of D1 (dashed box). For comparison, mature monomeric PSII (PDB-ID 3KZI) is shown in gray. (B) Superimposed 2D ${ }^{1} \mathrm{H}-{ }^{15} \mathrm{~N}-\mathrm{HSQC}$ spectra of free Psb28 (blue) and Psb28 bound to the Cterminal peptide of CP47 (magenta). Upper left inset: representation of slow exchange behavior for the proton amide resonance of T24, ranging from $126.9 \mathrm{ppm}$ to $128.6 \mathrm{ppm}$ in the ${ }^{15} \mathrm{~N}$ dimension. (C) CSPs of more than one SD projected onto the model representation of Psb28. (D) Weighted ${ }^{1} \mathrm{H} /{ }^{15} \mathrm{~N}$ chemical shift perturbations observed 
for Psb28 upon binding to the CP47 peptide. Red line indicates one standard deviation (SD), residues that yield resonances only in the complex form are indicated in orange. (E) Backbone ${ }^{15} \mathrm{~N}\left\{{ }^{1} \mathrm{H}\right\}$-heteronuclear NOE of free Psb28 (blue) and Psb28 bound to the C-terminal region of the CP47 peptide (magenta). Smaller I/I0 ratios correspond to regions that exhibit dynamics on the pico- to nanosecond timescale.

170

171

172

173

174

175

176

177

178

179

180

181

182

183

184

185

186

187

188

189

190

191

192

193

194

195

196

\section{Psb28 binding prevents full association of $\mathrm{CP43}$ and distorts the $Q_{\mathrm{B}}$ binding pocket}

Binding of Psb28 - with support of Psb34 - causes major structural perturbations at the PSII acceptor side (Supplementary Movies 1 and 2), which mainly involve the D-E loops of the central D1 and D2 subunits. Comparison of the CP43 structure in PSII-I with that in our PsbJ-free control PSII-M (Fig. 4A-D) or with that in mature monomeric PSII (PDB-ID 3KZI) (Fig. 4C and D) reveals several differences. The CP43 C-terminus is not resolved in PSII-I, probably due to an immature position of the last transmembrane helix of CP43 and an altered conformation of the D1 D-E loop, which may prevent binding of the CP43 C-terminus to the cytoplasmic PSII surface (Fig. 4B). This region is close to the loop between helices D and E of the D2 subunit, which is also altered by binding of Psb28, as clearly shown by movement of D2 Arg233 (Fig. 4B, Fig. S5A and B). After dissociation of Psb28, the CP43 module undergoes a rigid body rotation where it clicks into place (Fig. 4B-D, Supplementary Movie 1), whereas binding of PsbJ and the extrinsic proteins $\mathrm{PsbO}, \mathrm{PsbV}$ and $\mathrm{PsbU}$ during further maturation has very little influence on the CP43 binding position (Fig. 4C and D). The part of PSII that originates from RC47 shows almost no difference between PSII-I and mature PSII (Fig. 4D), except for PsbE, which binds adjacent to PsbJ (Fig. 4C).

Most importantly, the structural changes in the D1 D-E loop may have a direct functional impact on PSII electron transfer (Fig. 4E and F), as this region coordinates several important PSII cofactors. In functional PSII, after charge separation at the reaction center $\mathrm{P}_{680}$, electrons are transferred via pheophytin to the bound plastoquinone $\left(\mathrm{Q}_{\mathrm{A}}\right)$ and further to mobile plastoquinone (QB). In our PSII-I structure, the $\mathrm{Q}_{\mathrm{A}}$ site is fully assembled, and a well-resolved $\mathrm{Q}_{\mathrm{A}}$ molecule is bound (Fig. 4E and F, Fig. S5C and D). The nearby non-heme iron is also already in place in PSII-I (Fig. 4E and F, Fig. S5E and F). The $\mathrm{Q}_{\text {В }}$ binding site of the PSII-M control is comparable to mature PSII, although it is not occupied by $Q_{в}$ in our preparation (Fig. S5G). In contrast, the $\mathrm{Q}_{\text {в }}$ binding site of PSII-I is immature due to the Psb28- and Psb34-induced structural changes in the D1 D-E loop (Fig. 4E and F, Fig. S5H). Notably, D1 Phe265, which coordinates the head group of $\mathrm{Q}_{\mathrm{B}}$ in mature PSII, is clearly at a different position ${ }^{21}$ (Supplementary Movie 2). 
A

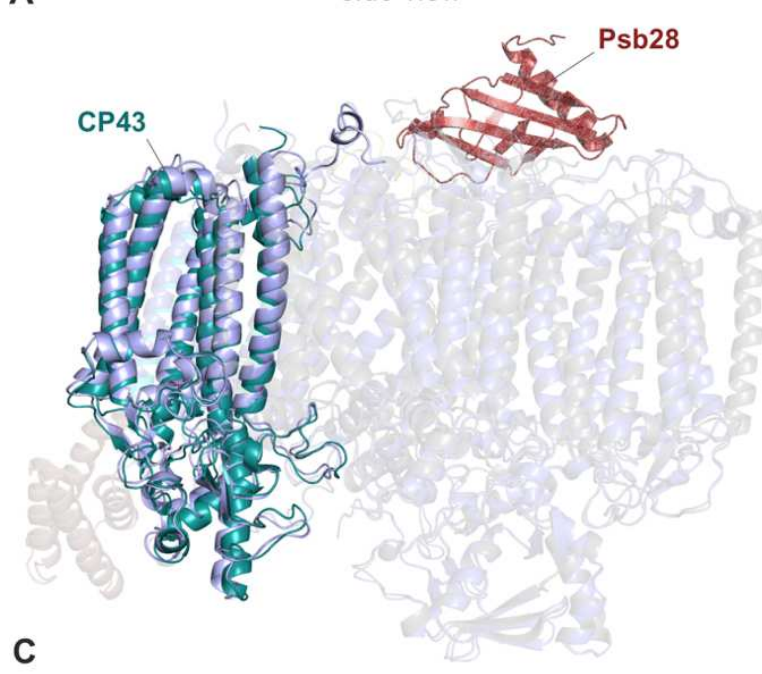

B

top view

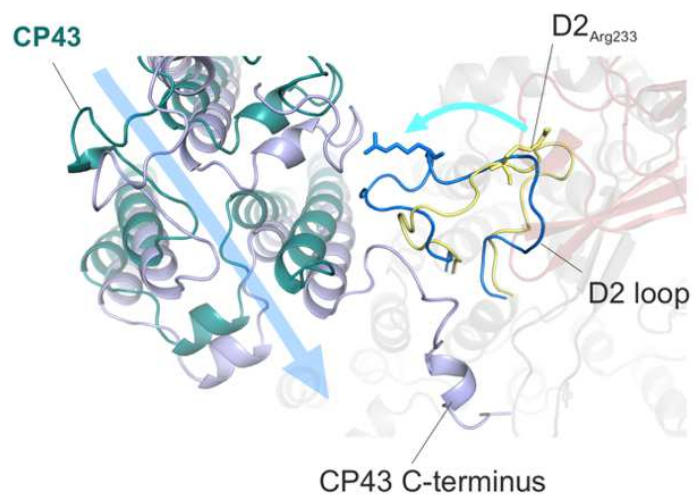

D

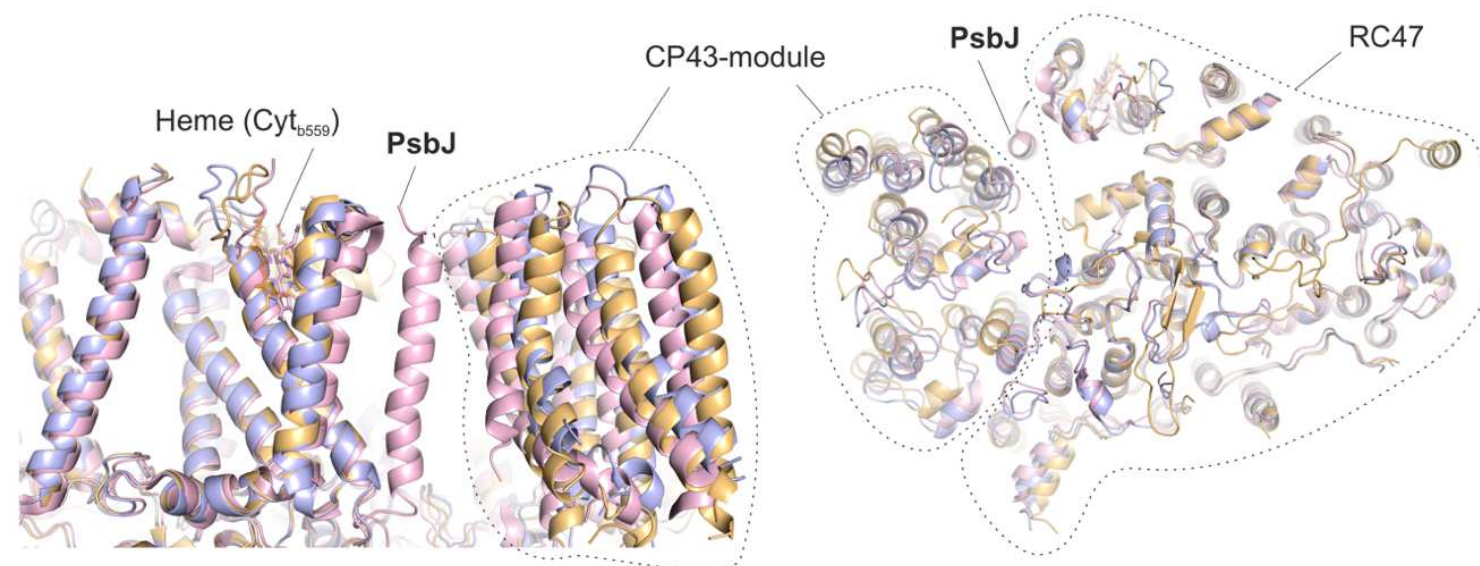

E

$\mathbf{F}$

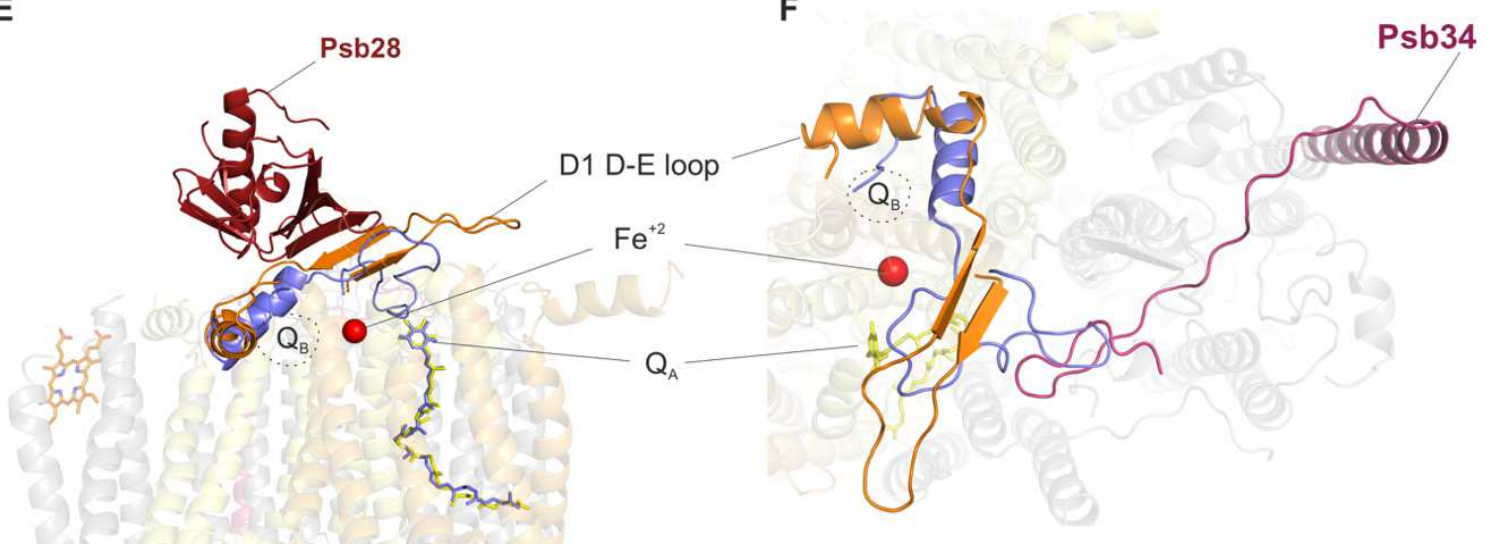

Fig. 4. Structural changes of the D1 and D2 D-E loops induced by binding of Psb28 and Psb34. (A) Side view of the CP43 antenna protein in PSII-I (teal) and the PSII-M control (light blue). (B) Structural changes between PSII-I and the PSII-M control in the cytoplasmic D2 D-E loop (yellow: PSII-I, blue: PSII-M) and attachment of CP43 (teal: PSII-I, light blue: PSII-M control) (top view). Details of the structural changes in the D2 loop are shown in Fig. S5A and B. (C) Side view and (D) top view of the PSII-I structure (orange) compared to the PSIIM control (light blue) and mature monomeric PSII (light red, PDB-ID 3KZI). (E) Side view and (F) top view of the Psb28-induced structural changes in the D1 D-E loop (orange) and perturbation of the Qв binding site compared to PSII-M (light blue), which lacks the assembly factors. $\mathrm{Q}_{\mathrm{A}}$ is shown in yellow (PSII-I) or light blue (PSII-M), respectively. See Fig. S5C-H for enlarged views of the $\mathrm{Q}_{A}$ and $\mathrm{Q}_{B}$ binding site and the adjacent nonheme iron. 
198 A more detailed analysis of the structural environment close to the $Q_{A} / Q_{B}$ binding sites revealed

199

200

201

202

203

204

205

206

207

208

209

210

211

212

213

214

215

216

217

218

219

220

221

222

223

224

225

226

227

228

229 differences in the coordination and the hydrogen-bond network of the adjacent non-heme iron, which also indicate functional consequences for PSII electron transfer and charge recombination processes. In mature PSII, the non-heme iron is coordinated by four histidine residues and bicarbonate as the fifth ligand (Fig. 5A and C), whereas in PSII-I, the bicarbonate molecule is replaced by the E241 side-chain of D2 (Fig. 5B and D, Fig. S5E and F, Supplementary Movie 3). Other residues, including D1 E244 and Y246, which bind to the bicarbonate molecule in mature PSII (Fig. 5A), are also displaced in PSII-I due to the conformational change of the D1 D-E loop (Fig. 5B, Fig. S5E and F, Supplementary Movie 3). Binding of bicarbonate is important for PSII efficiency ${ }^{47}$, as it lowers the redox potential of $\left(\mathrm{Q}_{\mathrm{A}} / \mathrm{Q}_{\mathrm{A}}{ }^{-}\right)$to favor forward electron transport ${ }^{48,49}$. If charge recombination occurs, the lower redox potential favors indirect charge recombination via $\mathrm{P}^{\bullet+} / \mathrm{Pheo}^{\bullet-}$. This back reaction yields triplet chlorophyll and subsequently singlet oxygen ${ }^{49}$, a highly oxidizing species. Changes in the redox potential of $\left(\mathrm{Q}_{\mathrm{A}} / \mathrm{Q}_{\mathrm{A}}^{-}\right)$have been proposed to tune the efficiency of PSII depending on the availability of $\mathrm{CO}_{2}$ as the final electron acceptor and thereby protect PSII under low $\mathrm{CO}_{2}$ conditions ${ }^{49}$. Therefore, we used flash-induced variable fluorescence to measure electron transfer in the PSII-I assembly intermediate and inactivated PSII, both of which lack a functional OEC (Fig. 5E, Fig. S6A and B). The fast component is assigned to PSII centers with fast reoxidation of $\mathrm{Q}_{\mathrm{A}}^{-}$by properly bound $\mathrm{Q}_{\mathrm{B}}$, the middle component is caused by PSII complexes with inaccurately bound $\mathrm{Q}_{\mathrm{B}}$, and the slow component is associated with centers that do not contain $\mathrm{Q}_{\mathrm{B}}$ and instead reoxidize $\mathrm{Q}_{\mathrm{A}}{ }^{-}$through charge recombination with the $\mathrm{Mn}_{4} \mathrm{CaO}_{5}$ cluster ${ }^{50}$. Fully functional PSII showed typical $\mathrm{Q}_{\mathrm{A}^{-}}$reoxidation, which is primarily due to fast electron transfer to $\mathrm{Q}_{\text {в }}$ (Fig. 5E, blue trace). Addition of DCMU blocks electron transfer to $\mathrm{Q}_{\text {в }}$ in active PSII, thereby promoting slow $\mathrm{S}_{2} \mathrm{Q}_{\mathrm{A}^{-}}$charge recombination (Fig. S6B, blue trace). Removal of the OEC increases the $\mathrm{Q}_{\mathrm{A}}$ redox potential ${ }^{51}$ and promotes very slow $\mathrm{Q}_{\mathrm{A}}{ }^{-} \mathrm{Tyr}_{\mathrm{D}}{ }^{+}$ recombination (Fig. 5E, black trace) ${ }^{52}$, which is influenced only minorly by binding of DCMU (Fig. S6B, black trace). PSII-I shows a different behavior (Fig. 5E, Fig. S6B red trace); $60 \%$ of the PSII-I centers decay within $1 \mathrm{~s}$, whereas $\sim 40 \%$ decay in PSII (-OEC). To determine whether the replacement of bicarbonate by glutamate affects the energetics of the redox couple $\mathrm{Q}_{\mathrm{A}} / \mathrm{Q}_{\mathrm{A}}{ }^{-}$, we measured the formation of ${ }^{1} \mathrm{O}_{2}$ by EPR spectroscopy using the spin probe TEMPD. The data clearly show that ${ }^{1} \mathrm{O}_{2}$ formation is reduced by $\sim 30 \%$ in PSII-I compared to inactivated PSII (-OEC), which contains bicarbonate (Fig. 5F). 


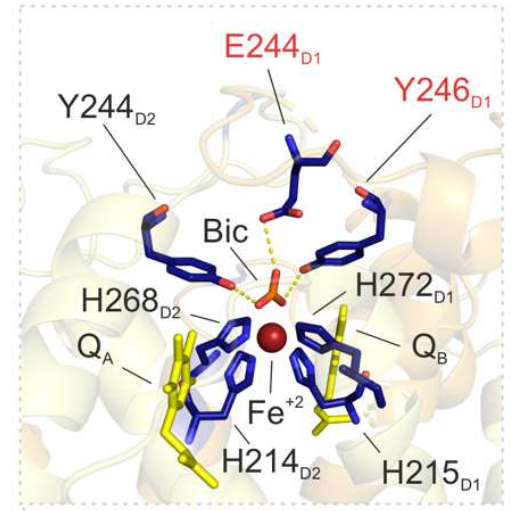

C

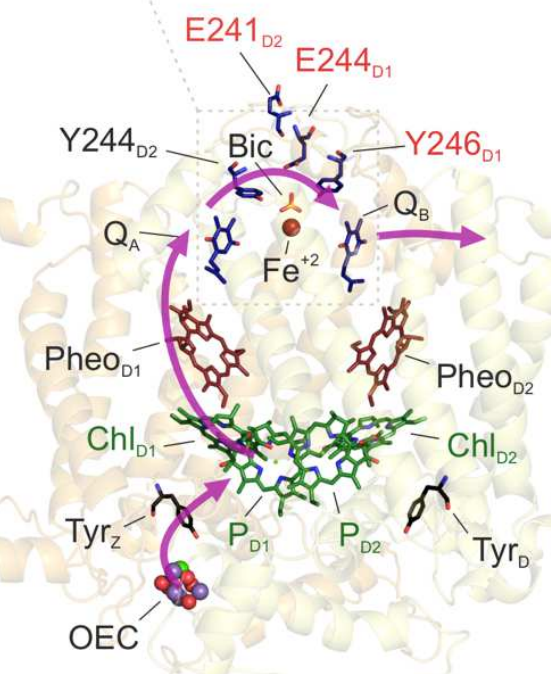

E

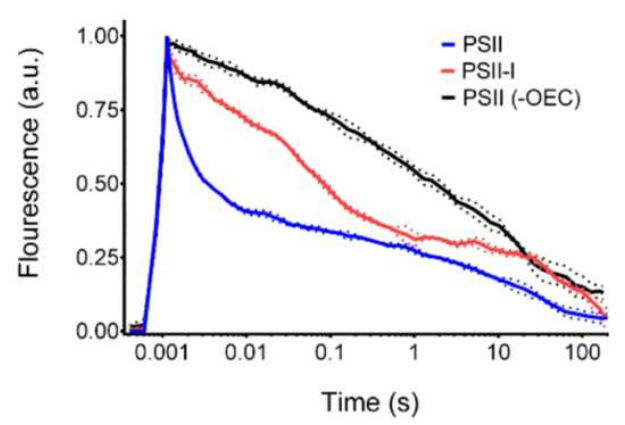

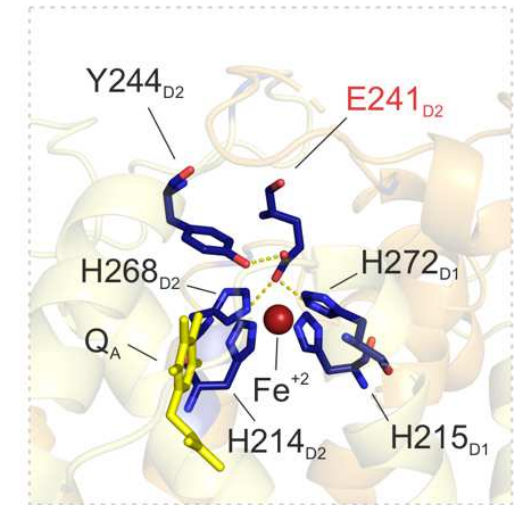

D

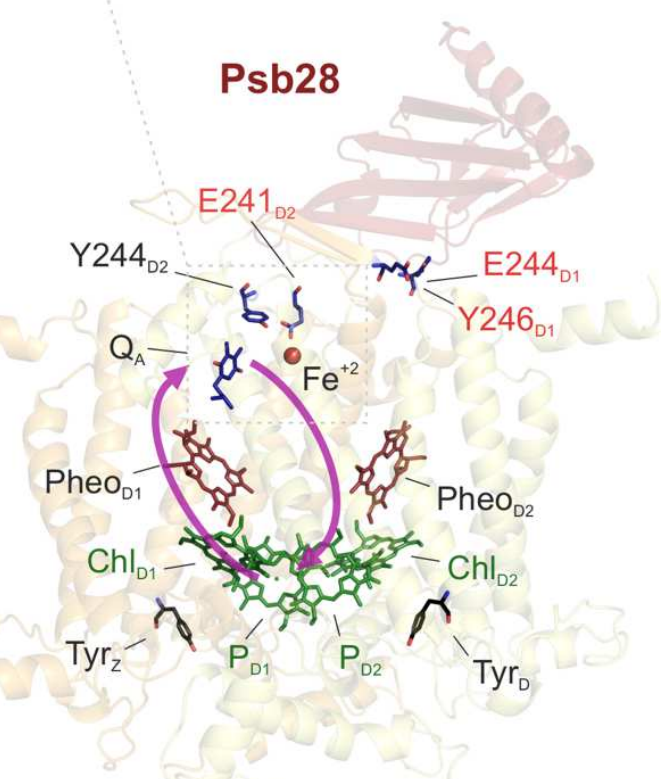

$\mathbf{F}$

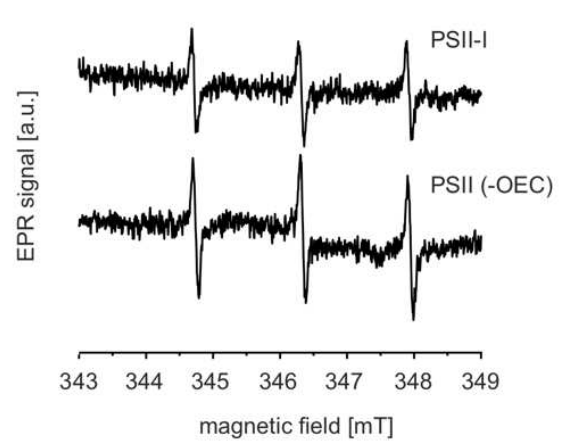

Fig. 5: Binding of Psb28 displaces bicarbonate as a ligand of the non-heme iron and protects PSII from damage. (A) The electron transfer from $\mathrm{PQ}_{\mathrm{A}}$ to $\mathrm{PQ}_{\mathrm{B}}$ is coordinated by the non-heme iron $\left(\mathrm{Fe}^{2+}\right)$, with the binding of bicarbonate (Bic) serving as a regulatory mechanism ${ }^{49}$ in mature PSII (PDB-ID 3WU2). (B) Binding of Psb28 to the PSII-I assembly intermediate induces a conformational change in the cytoplasmic D2 D-E loop, where the side chain of Glu241 replaces bicarbonate as a ligand of the non-heme iron. The respective fits of the non-heme iron binding sites are shown in Fig. S5E and F. A similar coordination is also found in non-oxygenic bacterial reaction centers ${ }^{53}$ (Fig. S6C). (C) Electron transfer (purple arrows) in mature PSII. Light-induced charge separation at the reaction center chlorophylls $\left(\mathrm{P}_{\mathrm{D} 1}, \mathrm{P}_{\mathrm{D} 2}, \mathrm{Ch}_{\mathrm{D} 1}, \mathrm{Ch} \mathrm{D}_{\mathrm{D} 2}\right)$ leads to electron transfer via pheophytin (PheoD1) and plastoquinone $\mathrm{A}\left(\mathrm{Q}_{\mathrm{A}}\right)$ towards $\mathrm{Q}_{\mathrm{B}}$. The electron gap at the reaction center is filled by the oxygen 
evolving complex (OEC). (D) Reoxidation of $\mathrm{Q}^{-}$by direct and safe charge recombination is favored in the PSII assembly intermediate, as indicated by the purple arrows. (E) Flash-induced fluorescence decay of PSII. Blue lines represent active PSII, black and red represent PSII without functional OEC and PSII-I respectively. Dotted corridors depict SD $(n=3)$. (F) The protective role of Psb28 binding was further confirmed by EPR spectroscopy using the spin probe TEMPD, which is specific for ${ }^{1} \mathrm{O}_{2}$, the major reactive oxygen species in PSII generated by triplet chlorophyll ( $\left.{ }^{3} \mathrm{P}\right)$. Inactivated PSII without functional $\mathrm{Mn}_{4} \mathrm{CaO}_{5}$ cluster (-OEC) was used as control.

\section{Psb27 binds in a remote position to loop E of CP43 at the luminal PSII surface}

Psb27 binds to the luminal side of the PSII complex, adjacent to loop E of the CP43 subunit (Fig. 6A). In contrast to previously proposed models ${ }^{54,55}$, the binding site of Psb27 has little overlap with the binding sites of the extrinsic subunits (PsbO, PsbV and PsbU) and has at least no direct impact on the $\mathrm{Mn}_{4} \mathrm{CaO}_{5}$ cluster binding site (Fig. 6A and B). Instead, Psb27 is bound at a remote position that might be occupied by CyanoQ in the mature complex ${ }^{56}$. This localization of Psb27 does not support previous functional models in which bound Psb27 prevents the binding of the extrinsic subunits or plays a direct role in $\mathrm{Mn}_{4} \mathrm{CaO}_{5}$ cluster assembly 33,54. However, Psb27 might stabilize loop E of CP43 in the unassembled state and facilitate its binding to the D1 subunit. This is of particular importance, as loop E of CP43 provides Arg345 and Glu342, two ligands of the $\mathrm{Mn}_{4} \mathrm{CaO}_{5}$ cluster in mature PSII (Fig. 6B, dashed box). Moreover, in the Psb27-bound state (PSII-I), the D1 C-terminus, which is directly involved in coordination of the $\mathrm{Mn}_{4} \mathrm{CaO}_{5}$ cluster $^{21}$, is bound away from the cluster (Fig. 6C, Fig. S7). Thus, our PSII-I structure reveals not only how the Psb27 protein binds to CP43 and thus stabilizes it prior to attachment, but also indicates an indirect role for CP43 in maturation of the oxygen evolving cluster that is consistent with functional data from previous studies $31,34,40$. 

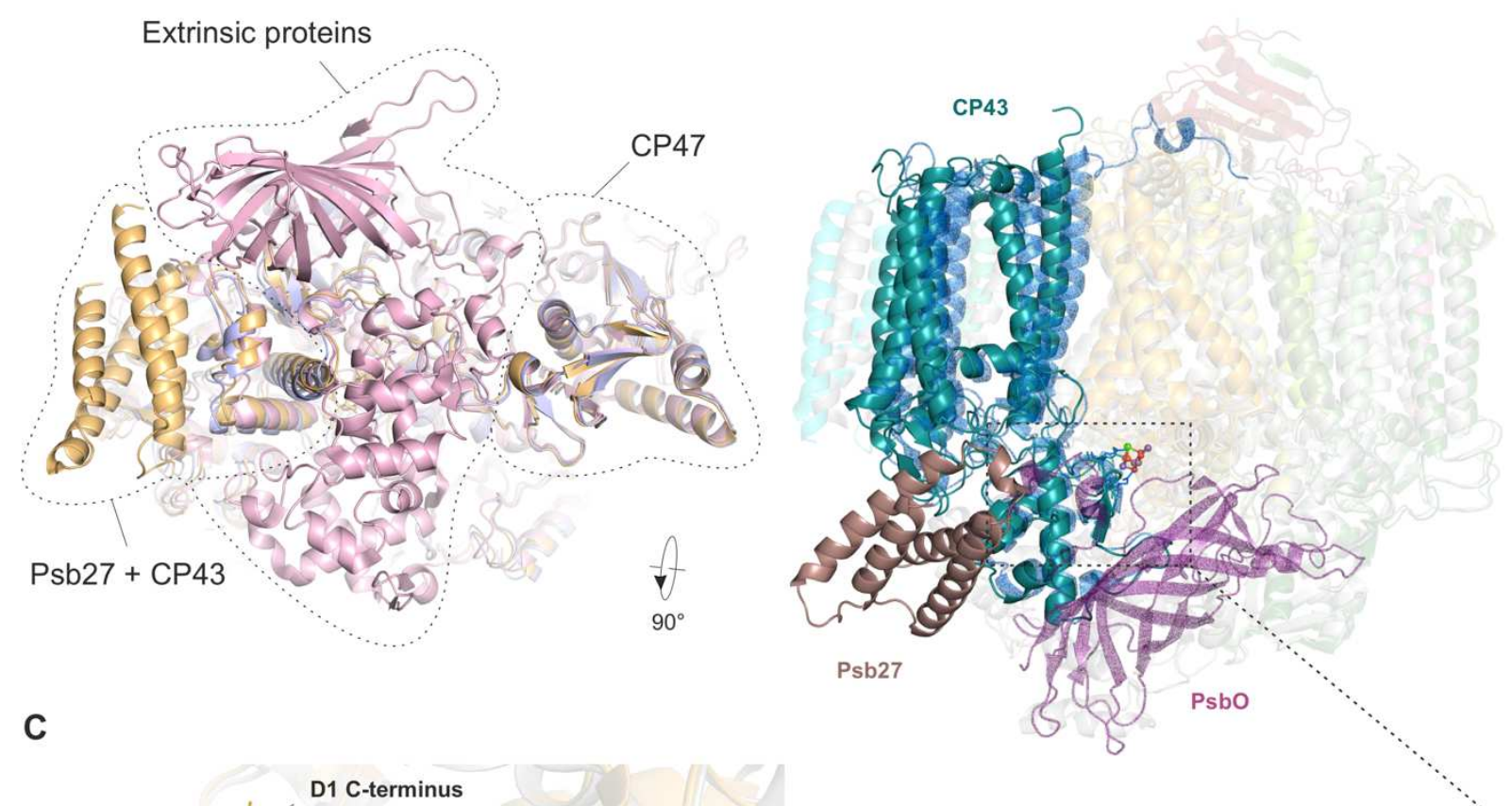

C
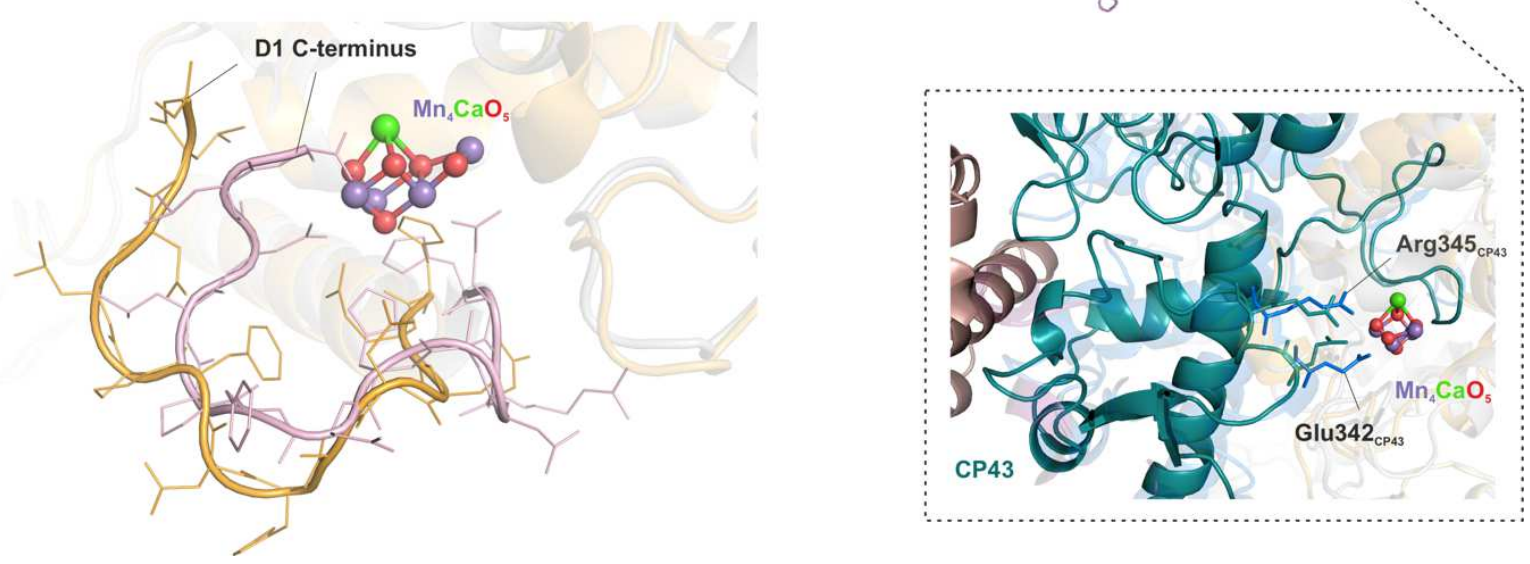

Fig. 6: The role of Psb27 in $\mathrm{Mn}_{4} \mathrm{CaO}_{5}$ cluster assembly. (A) Bottom view of the luminal PSII surface for PSIII (orange), the PSII-M control (light blue) and mature monomeric PSII (PDB-ID 3KZI) (light red). (B) Side view of CP43 (teal) and Psb27 (brown) in PSII-I, as well as of CP43 (blue) and PsbO (purple) in mature monomeric PSII (PDB-ID 3KZI). Dashed box: CP43 E loop with residues Arg345 and Glu342 (shown as sticks), which are involved in coordination of the $\mathrm{Mn}_{4} \mathrm{CaO}_{5}$ cluster. We changed the numbering of $\mathrm{CP} 43$ residues due to a corrected $\mathrm{N}$-terminal sequence (www.UniProt.org). The residues correspond to Arg357 and Glu354 in previous publications. The high-resolution structure of the $\mathrm{Mn}_{4} \mathrm{CaO}_{5}$ cluster is taken from Umena et al. 2011 (PDB-ID 3WU2). (C) Position of the D1 C-terminus in PSII-I (orange) and mature monomeric PSII (PDB-ID 3KZI) (light red).

The immature $\mathrm{Mn}_{4} \mathrm{CaO}_{5}$ cluster binding site of PSII-I contains a single, positively charged ion

248 The unique $\mathrm{Mn}_{4} \mathrm{CaO}_{5}$ cluster is a key feature of PSII that splits water into oxygen and protons.

249 However, our PSII-I complex does not show any oxygen-evolving activity, suggesting that the oxygen evolving complex (OEC) is not fully assembled. In mature PSII, the $\mathrm{Mn}_{4} \mathrm{CaO}_{5}$ cluster is submerged in the complex and additionally capped by the extrinsic subunits PsbO, PsbU and

252 PsbV (Fig. 6A and B). In our PSII-I structure, these subunits are absent, which leaves two parts of the CP43 E-loop (residues 320-327 and 397-404) in a flexible conformation, exposing the 
binding site of $\mathrm{Mn}_{4} \mathrm{CaO}_{5}$ cluster to the lumen. There is no strong density feature at this position that would correspond to the fully assembled metal-redox cofactor. Thus, our PSII-I structure provides a model for an immature OEC. By comparing our structure with the high-resolution crystal structure of mature PSII ${ }^{21}$ (PDB-ID 3WU2) provides insights into the first-steps of OEC biogenesis (Fig. 7).

259

260

The D1 C-terminus is one of the key features for the formation of the of OEC, as it provides several essential charged residues that are responsible for coordination of the chloride ion and the $\mathrm{Mn}_{4} \mathrm{CaO}_{5}$ cluster (Fig. 7A, $\mathrm{B}$ and D). The density for these $\mathrm{C}$-terminal residues is weak in our PSII-I map, but traceable (Fig. S7A), indicating a flexibility that confirms the absence of the OEC. Compared to the mature complex, the last 12 residues of the $\mathrm{C}$-terminal tail of D1 would need to undergo significant conformational changes to bring the side chains of Glu333, His337, Asp342, and the Ala344 C-terminus into the correct position to coordinate the $\mathrm{Mn}_{4} \mathrm{CaO}_{5}$ cluster (Supplementary Movie 4).

Moreover, we identify a clearly visible density at the position of the chloride ion, which is coordinated by Lys317 (D2) and the hydrogen atom of the backbone nitrogen of Glu333 (D1) in mature PSII (Fig. 7B and E). Despite the similar position, the $\mathrm{Cl}^{-}$is coordinated by the nitrogen atom of the ring of adjacent His332 (D1) in PSII-I (Fig. 7A and E, Fig. S7D). Surprisingly, we identified another density in the area where the $\mathrm{Mn}_{4} \mathrm{CaO}_{5}$ cluster is located in mature PSII (Fig. 7A-C and F, Fig. S7C). However, this density is not large enough to reflect the whole cluster. Based on its size and interaction partners (Fig. 7F), it corresponds to one positively charged ion. In the structural context, this ion is most likely $\mathrm{Mn}^{2+}$, but it could also be $\mathrm{Ca}^{2+}$ or any other positively charged ion.

This ion is coordinated by the side chains of D1 Asp170, Glu189, and His332, which are already in similar positions compared to mature PSII. Glu342 and Arg345 of CP43, which are both involved in the $\mathrm{Mn}_{4} \mathrm{CaO}_{5}$ cluster coordination, are also already pre-positioned through the interaction between Arg345 with D1 Asp170 (Fig. 7G). However, there are still significant conformational changes necessary for the transition from PSII-I to mature PSII, as highlighted in Figure 7D and G, as well as in Supplementary Movie 4. The D1 C-terminal tail must bring the side chains of Glu333, His337 and Asp342, as well as the C-terminus of Ala344, into correct alignment to coordinate the $\mathrm{Mn}_{4} \mathrm{CaO}_{5}$ cluster. In addition, the C-terminal tail of $\mathrm{D} 2$ needs to flip towards the D1 C-terminus (Fig. 7C, Fig. S7B, Supplementary Movie 4). In summary, PSII-I is characterized by only one positive charged ion bound instead of the complete $\mathrm{Mn}_{4} \mathrm{CaO}_{5}$ cluster, resulting in significantly different conformations of the D1 and D2 C-termini 
A

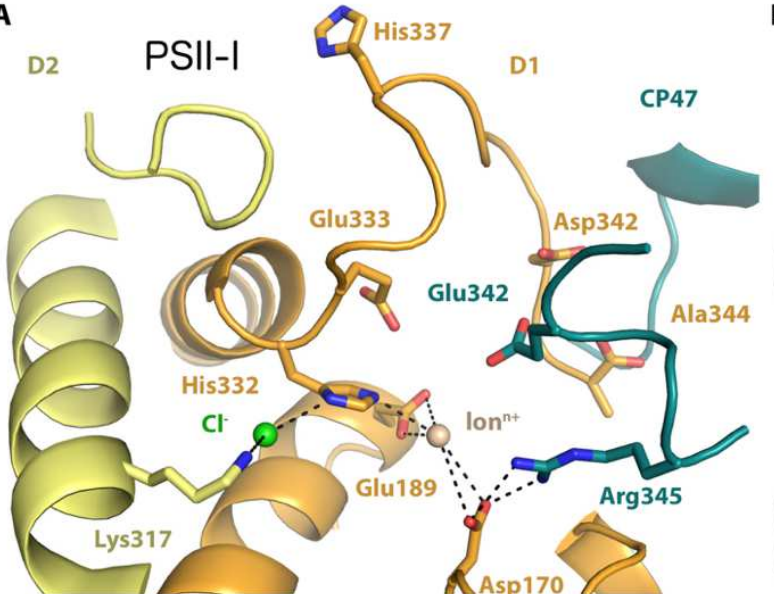

C D2

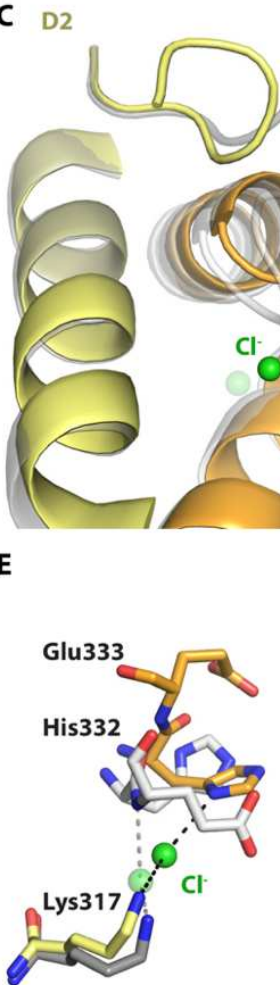

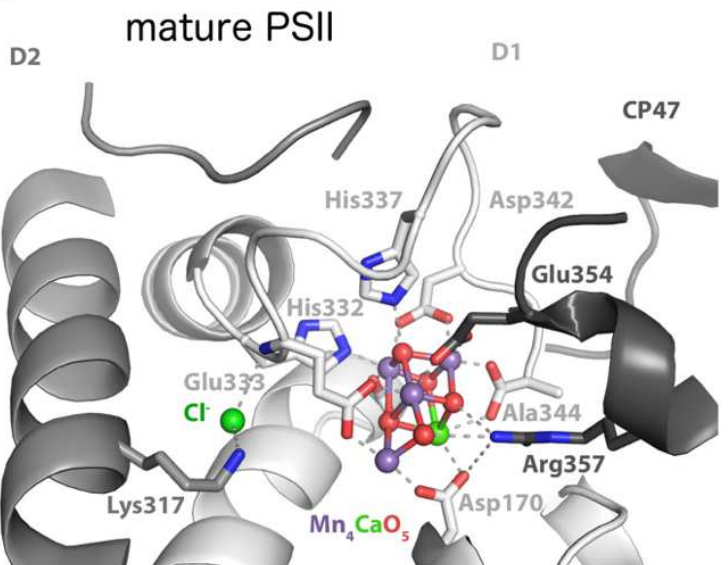

D

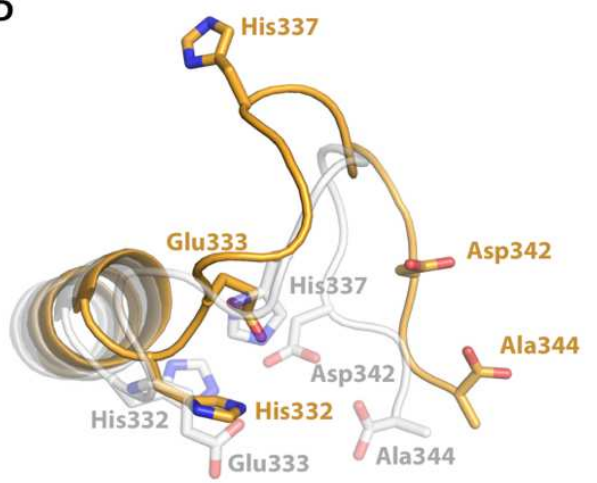

G

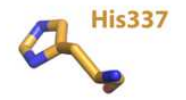

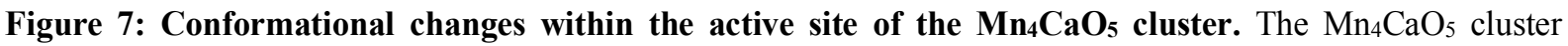
performs PSII's unique water-splitting reaction. (A) The active site of the $\mathrm{Mn}_{4} \mathrm{CaO}_{5}$ cluster is resolved within our PSII-I structural model but is not yet oxygen-evolving. (B) Crystal structure of the oxygen-evolving, mature PSII (PDB-ID 3WU2, resolution $1.9 \AA$ A). (C) Overlay of both structures, illustrating significant differences in the backbone conformation of the D1 and D2 C-terminal tails. (D) Accompanying side chain rearrangements of the D1 C-terminus. The $\mathrm{Cl}^{-}(\mathbf{E}), \mathrm{Ion}^{+}(\mathbf{F})$ and $\mathrm{Mn}_{4} \mathrm{CaO}_{5}(\mathbf{G})$ cluster coordination partners are compared in detailed. The validation of the fit to density for the structural details shown here is provided in Figure S7. 


\section{Discussion}

291 PSII biogenesis is a complex process that requires the action of specific assembly factors. These auxiliary proteins are not present in the mature complex and interact only transiently with specific subunits or preassembled PSII intermediates. Although more than 20 factors have been

294 identified and allocated to specific transitions, their precise molecular function in PSII assembly remains elusive in almost all cases. Our study provides the first detailed molecular insights into the function of PSII assembly factors Psb27, Psb28 and Psb34, which are involved in an important transition prior to activation of the OEC. The determined binding positions of Psb27 and Psb28, which are two of the most studied PSII assembly factors, disprove all previous Psb27 and Psb28 binding models and exclude Psb27 from direct involvement in OEC maturation ${ }^{54,55,57-61}$.

301 Binding of Psb28 and Psb34 to the cytoplasmic side of PSII induces large conformational changes in the D1 D-E loop (Fig. 4), which has been identified previously as an important location for PSII photoinhibition and D1 degradation ${ }^{62,63}$. Structural changes observed in the

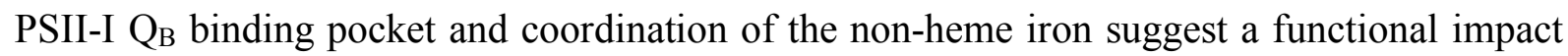
on PSII electron transfer to protect the immature complex until water splitting is activated. In particular, D2 Glu241 replacing bicarbonate as ligand of the non-heme iron by suggests a regulatory role, as binding of bicarbonate was proposed to tune PSII efficiency by changing the redox potential of $\left(\mathrm{Q}_{\mathrm{A}} / \mathrm{Q}_{\mathrm{A}}^{-}\right)^{48,49}$. As a functional consequence, PSII-I generates less singlet oxygen compared to inactive PSII (Fig. 5F).

Interestingly, the coordination of the non-heme iron in PSII-I resembles that in non-oxygenic bacterial reaction centers (BRCs) ${ }^{53}$ (Fig. S6C). In BRCs, the fifth ligand of the non-heme iron is provided by E234 of the M subunit ${ }^{64}$, and mutagenesis of this residue induces changes in the free energy gap between the $\mathrm{P}^{\bullet+} / \mathrm{Q}_{\mathrm{A}^{\bullet-}}$ radical pair ${ }^{65}$. These findings indicate that the environment of the non-heme iron is important for regulation of forward electron transfer to $Q_{B}$ versus charge recombination ${ }^{48}$. Therefore, we speculate that during biogenesis, PSII switches to a mechanism that usually operates in non-oxygenic bacterial reaction centers.

The Psb27-bound and -unbound structures do not differ substantially (Fig. 6B), suggesting a rather subtle action in PSII biogenesis. Previous work demonstrated that Psb27 is already bound to free $\mathrm{CP} 43^{31}$, where it might protect free $\mathrm{CP} 43$ from degradation or stabilize the E-loop in a specific conformation to chaperone the subsequent association with the RC47 complex. This step is crucial for preparing the binding site of the $\mathrm{Mn}_{4} \mathrm{CaO}_{5}$ cluster, as the CP43 E-loop provides two ligands of the cluster. Further OEC assembly is a multistep process that requires 
323 a functional upstream redox chain for the oxidation of $\mathrm{Mn}^{2+}$ to build up the cluster's $\mu$-oxo 324 bridges between the manganese atoms ${ }^{66-69}$. The mechanistic and structural details of this photoactivation process are not yet understood. In the consensus 'two quantum model', a single $\mathrm{Mn}^{2+}$ ion bound to the high-affinity site (HAS) is oxidized to $\mathrm{Mn}^{3+}$. This initiating light-

327 dependent step is followed by a slow light-independent phase and further fast light-dependent steps in which the remaining $\mathrm{Mn}^{2+}$ ions are oxidized and incorporated. Understanding the lightindependent slow phase is key to unraveling the mechanism of photoactivation.

330 Previous structural studies aimed to obtain mechanistic insights into the dark-rearrangement by removing the $\mathrm{Mn}_{4} \mathrm{CaO}_{5}$ cluster from fully assembled PSII, either by depleting it directly from PSII crystals by chemical treatment ${ }^{70}$ or by cryo-EM single particle analysis in manganese- and calcium-free buffer ${ }^{71}$. The X-ray structure was indeed missing the $\mathrm{Mn}_{4} \mathrm{CaO}_{5}$ cluster, but the D1 C-terminus followed mostly the same trajectory as found in the mature PSII-dimer structure. The authors suggested that the D1 C-terminus might not rearrange during $\mathrm{Mn}_{4} \mathrm{CaO}_{5}$ cluster assembly. However, the crystal structure was dimeric and still had the extrinsic subunits PsbO, PsbU, and PsbV bound. It is known that these subunits are typically not associated with $\mathrm{Mn}_{4} \mathrm{CaO}_{5}$ cluster-depleted PSII. Thus, the structure might be artificially stabilized by crystal packing forces. The cryo-EM structure, on the other hand, revealed a monomeric PSII that lacks extrinsic subunits and the $\mathrm{Mn}_{4} \mathrm{CaO}_{5}$ cluster $^{71}$. This structure is more similar to our PSII biogenesis intermediate PSII-I, as PsbY, PsbZ and PsbJ are also missing. The PsbJ subunit is surprising; it is an integral subunit of PSII and should not be easily detached, yet it is missing from this structure and we deleted it to stabilize our PSII-I complex. These observations might indicate a more specific and regulatory role of PsbJ in PSII biogenesis. Additionally, the D1 Cterminus is disordered in this previous cryo-EM structure, and the authors suggest that the darkrearrangement involves a transition from a disordered to an ordered state.

347 Our structure now reveals the fate of the D1 C-terminus with the assembly factor Psb27 bound. The D1 C-terminus follows a different trajectory compared to the mature PSII. Thus, we provide structural evidence that the slow dark-rearrangement involves a conformational change of the D1 C-terminus rather than the previously proposed disorder-to-order transition after initial photoactivation ${ }^{71}$. Compared to mature PSII, twelve residues of the D1 C-terminal tail must undergo significant conformational changes to bridge the side chains of Glu333, His337 and Asp342, as well as to bring the C-terminus of Ala344 in the correct position to coordinate the $\mathrm{Mn}_{4} \mathrm{CaO}_{5}$ cluster (Fig. 6C and 7D, Supplementary Movie 4), which is consistent with previous models ${ }^{69,72,73}$. We also identified a single positively charged ion in our PSII-I structure, coordinated by Asp170, Glu189 and His332 of D1 (Fig. 7F), at the position of the 
$357 \mathrm{Mn}_{4} \mathrm{CaO}_{5}$ cluster of mature PSII. This binding site most likely corresponds to the long-sought 358 single high-affinity site (HAS), where the first $\mathrm{Mn}^{2+}$ binds prior to the first photoactivation step 359 in OEC biogenesis ${ }^{74}$. However, we cannot exclude binding of $\mathrm{Ca}^{2+}$, which was shown to bind 360 with a much lower affinity ${ }^{72,75}$, or any other positive charged ion at this position. Nevertheless, 361 Asp170 has been identified as the most critical residue for the HAS ${ }^{76,77}$, which supports our 362 hypothesis. Further photoactivation steps occur presumably after cooperative binding of 363 calcium and manganese. The binding of the extrinsic subunit PsbO, potentially after release of 364 Psb27 and maturation of the WOC, is the next step of the PSII assembly line in vivo, which 365 leads to the next unsolved question in PSII biogenesis: what triggers the release of an assembly 366 factor? For Psb27, its detachment might be promoted by the binding of PsbO, as their binding 367 sites partially overlap.

368 Membrane protein complexes play a fundamental role in bioenergetics to sustain and proliferate 369 life on Earth. They drive the light-to-chemical energy conversion in photosynthetic organisms 370 and are essential for energy supply in heterotrophs. These highly complex molecular machines 371 are assembled from numerous single proteins in a spatiotemporally synchronized process that 372 is facilitated by a network of assembly factors. These auxiliary proteins are the key players of 373 Nature's assembly lines. Our PSII-I cryo-EM structure reveals the first molecular snapshot of 374 PSII biogenesis and, accompanied by our spectroscopic and biochemical analyses, provides 375 clear mechanistic insights into how three assembly factors (Psb27, Psb28 and Psb34) coordinate 376 the stepwise construction of this powerful catalyst of life. 


\section{Cultivation of Thermosynechococcus elongatus BP-1}

379 Cell growth and thylakoid membrane preparation were performed as described previously ${ }^{78}$. In 380 brief, $T$. elongatus mutant strains ( $\Delta$ psbJ psbC-TS and psb34-TS) were grown in BG-11 liquid 381 medium inside a 25-liter foil fermenter (Bioengineering) at $45^{\circ} \mathrm{C}, 5 \%(\mathrm{v} / \mathrm{v}) \mathrm{CO}_{2}$-enriched air bubbling and 50-200 $\mu \mathrm{mol}$ photons $\mathrm{m}^{-2} \mathrm{~s}^{-1}$ white light illumination (depending on the cell density). Cells were harvested at an $\mathrm{OD}_{680}$ of $\sim 2$ after 5-6 days of cultivation and concentrated to $\sim 0.51$ using an Amicon DC10 LA hollow fiber system, pelleted (3500 rcf, 45 min and 25 $\left.{ }^{\circ} \mathrm{C}\right)$ and resuspended in $150 \mathrm{ml}$ of Buffer D (100 mM Tris-HCL, pH 7.5, $10 \mathrm{mM} \mathrm{MgCl}_{2}, 10$ $\mathrm{mM} \mathrm{CaCl}_{2}, 500 \mathrm{mM}$ mannitol and 20\% (w/v) glycerol). The harvested cells were flash frozen in liquid nitrogen and stored at $-80^{\circ} \mathrm{C}$ until further use.

\section{Preparation of $T$. elongatus mutant strains}

Thermosynechococcus elongatus $\Delta \mathrm{psbJ}$ psbC-TS was generated based on the previously described strain $T$. elongatus $\Delta \mathrm{psbJ}^{35}$ that was transformed with the plasmid pCP43-TS. The plasmid is based on pCP34-10His ${ }^{33}$. The His-tag sequence was exchanged with TwinStrep-tag by PCR using the primers CP43TS_rev (5'CCCGATATCTTACTTCTCAAATTGCGGAT GAGACCACGCAGAACCACCAGAACCACCGCCGCTGCCGCCGCCTTTTTCGAACTG CGGGTGGCTCC 3') and NTCP43 (5' TGCTCTAGAATGAAAACTTTGTCTTCCCAGA $\left.3^{\prime}\right)$. The resulting PCR product was ligated back into an empty pCP34-10His backbone using $\mathrm{XbaI}$ and EcoRV restriction endonucleases. T. elongatus BP-1 cells were transformed as described previously ${ }^{79}$. Mutant colonies were selected by frequent re-plating onto agar plates with increasing antibiotic concentrations, stopping at $8 \mu \mathrm{g} / \mathrm{ml}$ of chloramphenicol and $80 \mu \mathrm{g} / \mathrm{ml}$ of kanamycin. Complete segregation of the mutant was confirmed by PCR with the primers CTCP43DS (5' CCGCTCGAGACCATCCAAGCTTGGCAGCA 3') and NTCP43 (5' TGCTCTAGAATGAAAACTTTGTCTTCCCAGA 3'). T. elongatus psb34-TS was generated by transformation with the plasmid pPsb34-TS. The plasmid DNA was obtained from TwistBioscience. It consisted of psb34 (ts10063) with a C-terminal TwinStrep-tag and a kanamycin resistance cassette, flanked by ts10063-upstream and downstream regions (900 bp each). T. elongatus BP-1 cells were transformed ${ }^{79}$ and mutant selection took place ${ }^{33}$. Complete segregation of the mutant was verified by PCR. The primers used were ts10063-up-for ( $5^{\prime}$ CATATGGTCTCGCAATTATTTGCCCATGC 3') and ts10063-down-rev (5' GGTACCCCG ACACAGTTGATCACCGC 3'). 
410 Thawed cells were diluted in $100 \mathrm{ml}$ of Buffer A (100 mM Tris-HCL, pH 7.5, $10 \mathrm{mM} \mathrm{MgCl}_{2}$

411

412

413

414

415

416

417

418

419

420

421

422

423

424

425

426

427

428

429

430

431

432

433

434

435

436

437

438

439

440

441

and $\left.10 \mathrm{mM} \mathrm{CaCl}_{2}\right)$ and pelleted again $\left(21000 \mathrm{rcf}, 20 \mathrm{~min}\right.$ and $\left.4^{\circ} \mathrm{C}\right)$. The pellet was resuspended in $100 \mathrm{ml}$ of Buffer A with $0.2 \%$ (w/v) lysozyme and dark incubated for $75-90 \mathrm{~min}$ at $37{ }^{\circ} \mathrm{C}$. This was followed by cell disruption by Parr bomb (Parr Instruments Company) and pelleting (21 $000 \mathrm{rcf}, 20 \mathrm{~min}$ and $4^{\circ} \mathrm{C}$ ). All following steps were performed under green illumination. The pellet was resuspended in $150 \mathrm{ml}$ of Buffer A and pelleted again (21 $000 \mathrm{rcf}, 20 \mathrm{~min}$ and $\left.4^{\circ} \mathrm{C}\right)$. This step was repeated three times, with the last resuspension in $80 \mathrm{ml}$ of Buffer B (100 $\mathrm{mM}$ Tris-HCL, $\mathrm{pH} 7.5,10 \mathrm{mM} \mathrm{MgCl}_{2}, 10 \mathrm{mM} \mathrm{CaCl}_{2}$ and $500 \mathrm{mM}$ mannitol). The isolated thylakoids were flash frozen in liquid nitrogen and stored at $-80^{\circ} \mathrm{C}$.

Strep-Tactin-affinity purification of PsbC-TS and Psb34-TS assembly intermediates were performed under green illumination. Membrane protein extraction was performed as described previously $^{78}$, with certain adaptations. Thylakoid membranes were supplemented with $0.05 \%$ $(\mathrm{w} / \mathrm{v}) \mathrm{n}$-Dodecyl $\beta$-maltoside (DDM) (Glycon) and pelleted $\left(21000 \mathrm{rcf}, 20 \mathrm{~min}\right.$ and $\left.4^{\circ} \mathrm{C}\right)$. The sample was resuspended in extraction buffer (100 mM Tris-HCL, pH 7.5, $10 \mathrm{mM} \mathrm{MgCl}_{2}, 10$ $\mathrm{mM} \mathrm{CaCl}_{2}, 1.2 \%(\mathrm{w} / \mathrm{v}) \mathrm{DDM}, 0.5 \%(\mathrm{w} / \mathrm{v})$ sodium-cholate and $\left.0.01 \%(\mathrm{w} / \mathrm{v}) \mathrm{DNase}\right)$ to a final chlorophyll concentration of $1 \mathrm{mg} / \mathrm{ml}$ and incubated for $30 \mathrm{~min}$ at $20^{\circ} \mathrm{C}$. The solubilized membrane proteins were ultra-centrifugated (140000 rcf, $60 \mathrm{~min}$ and $4{ }^{\circ} \mathrm{C}$ ) and $\mathrm{NaCl}$ was added to the supernatant to a final concentration of $300 \mathrm{mM}$.

The supernatant was filtered through a $0.45 \mu \mathrm{m}$ filter and applied to a $5 \mathrm{ml}$ Strep-Tactin Superflow HC column (IBA Lifesciences), equilibrated in Buffer W (100 mM Tris-HCL, pH 7.5, $10 \mathrm{mM} \mathrm{MgCl}_{2}, 10 \mathrm{mM} \mathrm{CaCl}_{2}, 500 \mathrm{mM}$ mannitol, $300 \mathrm{mM} \mathrm{NaCl}$ and $0.03 \%$ (w/v) DDM) at a flowrate of $3 \mathrm{ml} / \mathrm{min}$. The column was washed with Buffer $\mathrm{W}$ until a stable baseline $\left(\mathrm{A}_{280}\right)$ was reached. Strep-tagged protein complexes were eluted by an isocratic elution with Buffer E (100 mM Tris-HCL, pH 7.5, 10 mM MgCl $2,10 \mathrm{mM} \mathrm{CaCl}_{2}, 500 \mathrm{mM}$ mannitol, $300 \mathrm{mM} \mathrm{NaCl}$ $2.5 \mathrm{mM}$ desthiobiotin and $0.03 \%(\mathrm{w} / \mathrm{v}) \mathrm{DDM})$. The captured fractions were equilibrated in Buffer F (20 mM MES, pH 6.5, $10 \mathrm{mM} \mathrm{MgCl}_{2}, 10 \mathrm{mM} \mathrm{CaCl}_{2}, 500 \mathrm{mM}$ mannitol and 0.03\% $(\mathrm{w} / \mathrm{v}) \mathrm{DDM}$ ) with a spin concentrator (Amicon, Ultra - 15, 100000 NMWL), flash-frozen in liquid nitrogen and stored at $-80{ }^{\circ} \mathrm{C}$ until analysis.

PsbC-TS containing assembly intermediates were further separated by ion exchange chromatography (IEC). Captured elution fraction from the Strep-Tactin-affinity purification were loaded onto a anion exchange column (UNO Q-6, Biorad) with a flowrate of $4 \mathrm{ml} / \mathrm{min}$, pre-equilibrated in Buffer F. Protein complexes were eluted by a liner gradient of $\mathrm{MgSO}_{4}(0-$ 
443 mannitol, $150 \mathrm{mM} \mathrm{MgSO}_{4}$ and $0.03 \%(\mathrm{w} / \mathrm{v})$ DDM). Fractions containing PSII assembly 444 intermediates were collected, concentrated to $100-10 \mu \mathrm{M}$ reaction centers, using a spin concentrator (Amicon, Ultra - 15, 100000 NMWL), aliquoted, flash frozen in liquid nitrogen and stored at $-80{ }^{\circ} \mathrm{C}$ until further analysis.

\section{Protein Expression and Purification of Psb28}

448

449

450

451

452

453

454

455

The Psb28 expression plasmid was constructed by first amplifying psb28 from T. elongatus genomic DNA, using primers TeloPsb28for (5' GGAATTCCATATGGGTGCAATGGCA GAAATTC 3') and TeloPsb28rev (5' CGAATTCCCCGGGAGAGTTCTCAGACTTCTG 3'). Next, the amplified DNA was cloned into pIVEX2.3d using NdeI/SmaI to obtain pIVEXPsb28His. Expression and purification of ${ }^{15} \mathrm{~N}$-labelled Psb28 was carried out as described previously ${ }^{80}$ with certain adaptations. Overnight starter cultures were grown on agar plates at $37{ }^{\circ} \mathrm{C}$, supplemented with $1 \%$ (w/v) glucose and $100 \mu \mathrm{g} / \mathrm{ml}$ ampicillin. The cell material was then resuspended in $2 \mathrm{ml}$ of M9 media ${ }^{81}$, and this was used to inoculate $500 \mathrm{ml}$ of M9 media with ${ }^{15} \mathrm{NH}_{4} \mathrm{Cl}$ as the only nitrogen source. Cultures were incubated at $37{ }^{\circ} \mathrm{C}$ under vigorous shaking and at an $\mathrm{OD}_{600}$ of 0.6 , and expression was induced with addition of isopropylthiogalactoside to a final concentration of $0.5 \mathrm{mM}$. After overnight incubation the ${ }^{15} \mathrm{~N}$ labbled Psb28 was isolated and purified as described for his-tagged $\mathrm{CyanoP}^{80}$. The purity and integrity of the protein samples were checked by SDS-PAGE (data not shown).

\section{Polyacrylamide Gel Electrophoresis}

Blue-native PAGE ${ }^{82}$ was used to assess the oligomeric state of the isolated PSII assembly intermediates. Separation of protein complexes was carried out across a linear gradient of polyacrylamide (acrylamide-bisacrylamide, 32:1) from 3.2 to $16 \%(\mathrm{w} / \mathrm{v})$ in the separating gel. This was overlaid with a $3 \%(\mathrm{w} / \mathrm{v})$ polyacrylamide (acrylamide-bisacrylamide, 32:1) sample gel. The gels were loaded with $40 \mu \mathrm{g}$ of protein per lane. Electrophoresis was performed at 4 ${ }^{\circ} \mathrm{C}$ in a Mini-PROTEAN Tetra System (BioRad) at $100 \mathrm{~V}$ for $30 \mathrm{~min}$ with Blue Cathode Buffer (15 mM BisTris-HCl, pH 7.0, 50 mM Tricine and 0.002\% (w/v) Coomassie Brilliant Blue 250) and at $170 \mathrm{~V}$ for an additional 90 min with Cathode Buffer (15 mM BisTris-HCl, pH 7.0 and $50 \mathrm{mM}$ Tricine). The anode buffer was composed of $50 \mathrm{mM}$ BisTris- $\mathrm{HCl}$ at $\mathrm{pH} 7$.

Subunit composition was investigated by SDS-PAGE ${ }^{83}$. Separation of polypeptide chains took place on a $19 \%(\mathrm{w} / \mathrm{v})$ polyacrylamide gel (acrylamide-bisacrylamide, 37.5:1), containing $9 \mathrm{M}$ urea and $4 \%(\mathrm{w} / \mathrm{v})$ glycerol. The gel was loaded with $40 \mu \mathrm{g}$ of denatured protein complex per lane. The gels ran at $4{ }^{\circ} \mathrm{C}$ in a Mini-PROTEAN Tetra System (BioRad) at $35 \mathrm{~mA}$ per gel for 60 
min. Fixation and visualization of polypeptide chains was performed with Coomassie Staining Solution $(45 \%(\mathrm{w} / \mathrm{v})$ isopropanol, $10 \%(\mathrm{w} / \mathrm{v})$ acetate and $0.2 \%(\mathrm{w} / \mathrm{v})$ Coomassie Brilliant Blue 250).

\section{Mass spectrometry analysis}

PSII-I complexes were purified and desalted using Isolute C18 SPE cartridges (Biotage, Sweden). The columns were first washed and equilibrated, the sample diluted in $0,1 \%$ trifluoroacetic acid (TFA) and loaded onto the column. After washing with $2 \mathrm{ml} \mathrm{0.1 \%} \mathrm{TFA,} \mathrm{the}$ proteins were eluted with $500 \mu 180 \%$ acetonitrile (ACN), 20\% water. The organic fraction was lyophilized in a vacuum concentrator (Eppendorf, Germany), reconstituted in $0.1 \%$ TFA and mixed in a 1:1 ratio with HCCA matrix solution (HCCA (alpha-cyano-4-hydroxycinnamic acid) saturated in 50\% ACN, 50\% water and supplemented with $0.1 \%$ TFA). Subsequently, 1 $\mu 1$ aliquots of the mixture were deposited on a ground steel MALDI target and allowed to dry and crystallize at ambient conditions.

MS and MS/MS spectra were acquired on a prototype rapifleX MALDI-TOF/TOF (Bruker Daltonics, Germany) in positive ion mode. The Compass 2.0 (Bruker Daltonics, Germany) software suite was used for spectra acquisition and processing (baseline subtraction, smoothing, peak picking), a local Mascot server (version 2.3, Matrixscience, UK) was used for database searches against the T. elongatus proteome (UniProt, retrieved 4/2019) and BioTools 3.2 (Bruker Daltonics) was used for manual spectrum interpretation, de novo sequencing and peak annotation.

\section{Flash-induced fluorescence decay measurements}

Flash-induced fluorescence decay was measured on a FL3500 Dual-Modulation Kinetic Fluorometer (PSI Photon Systems Instruments). Reaction centers were exited with $625 \mathrm{~nm}$ LEDs for both actinic $(50 \mu \mathrm{s})$ and measuring flashes. The first data point was collected $80 \mu \mathrm{s}$ after the actinic flash. Data points were collected from $80 \mu \mathrm{s}$ to 50 or $200 \mathrm{~s}$ after the actinic flash for measurements with whole cells and isolated PSII, respectively. 10 data points were collected per logarithmic decade. Assays were performed at room temperature in the presence and absence of 20, 100, 200 or $400 \mu \mathrm{M}$ 3-(3,4-dichlorophenyl)-1,1-dimethylurea (DCMU) with 5 min of dark incubation prior to measurement. Assays with isolated PSII complexes were carried out with $200 \mathrm{nM}$ reaction centers in activity buffer $(100 \mathrm{mM} \mathrm{KCl}, 20 \mathrm{mM}$ MES-KOH, pH 6.5, $10 \mathrm{mM} \mathrm{MgCl}_{2}, 10 \mathrm{mM} \mathrm{CaCl}_{2}$ and $0.03 \%$ (w/v) DDM). 
507 Typically, NMR samples contained up to $1 \mathrm{mM}$ of protein in $20 \mathrm{mM}$ Tris/ $\mathrm{HCl} \mathrm{pH} 8,10 \% \mathrm{D}_{2} \mathrm{O}$, $5080.02 \% \mathrm{NaN}_{3}$, and DSS. NMR spectra were acquired at $298 \mathrm{~K}$ on Bruker DRX 600 and AVANCE III HD 700 spectrometers. Backbone assignments for the free form of Psb28 were obtained from three-dimensional $\mathrm{HNCA}^{84,85}$, and $\mathrm{CBCA}(\mathrm{CO}) \mathrm{NH}^{86}$ spectra. Side-chain assignments were obtained from three-dimensional ${ }^{1} \mathrm{H}-{ }^{15} \mathrm{~N}$ HNHA ${ }^{87},{ }^{1} \mathrm{H}-{ }^{13} \mathrm{C}-\mathrm{HCCH}-\mathrm{TOCSY}$ ${ }^{88},{ }^{1} \mathrm{H}_{-}{ }^{15} \mathrm{~N}-\mathrm{HSQC}-\mathrm{TOCSY}{ }^{89},{ }^{1} \mathrm{H}-{ }^{15} \mathrm{~N}-\mathrm{HSQC}-\mathrm{NOESY}{ }^{90},{ }^{1} \mathrm{H}-{ }^{13} \mathrm{C}-\mathrm{HSQC}-\mathrm{NOESY}{ }^{91}$, and aromatic ${ }^{1} \mathrm{H}-{ }^{13} \mathrm{C}-\mathrm{HSQC}-\mathrm{NOESY}$ spectra. Spectra were processed with NMRPipe ${ }^{92}$ and analysed with CcpNmr Analysis ${ }^{93}$. NMR experiments for the complex form of Psb28 and the C-terminal peptide of CP47 were carried out on a Bruker AVANCE III HD 700 spectrometer at $298 \mathrm{~K}$ in $20 \mathrm{mM}$ Tris/ $\mathrm{HCl} \mathrm{pH} 8,10 \% \mathrm{D}_{2} \mathrm{O}, 0.02 \% \mathrm{NaN}_{3}$, and DSS. For the backbone assignments of the complex, a $1 \mathrm{mM}$ sample of $\left[\mathrm{U}^{-15} \mathrm{~N}^{13} \mathrm{C}\right]$-enriched $\mathrm{Psb} 28$ was mixed with a three-fold excess of peptide. Three-dimensional (3D) $\mathrm{HNCA}, \mathrm{HNCO}^{84,85}, \mathrm{HN}(\mathrm{CO}) \mathrm{CACB}^{94}, \mathrm{HNCACB}^{95,96}$, and $\mathrm{HN}(\mathrm{CA}) \mathrm{CO}^{97}$ were recorded with 16 scans and $25 \%$ non-uniform sampling (NUS). (H)CC(CO)NH and $\mathrm{H}(\mathrm{CCCO}) \mathrm{NH}$ spectra ${ }^{98,99}$ were recorded with 64 scans and $25 \%$ NUS. HNHA and ${ }^{1} \mathrm{H}^{15} \mathrm{~N}-\mathrm{HSQC}-\mathrm{NOESY}$ spectra were recorded with 32 scans and $25 \%$ NUS as well as traditional acquisition schemes, respectively. The mixing time for NOESY spectra was set to $120 \mathrm{~ms}$. In addition, heteronuclear two-dimensional ${ }^{15} \mathrm{~N}\left\{{ }^{1} \mathrm{H}\right\}$-NOE data were recorded in order to extract pico- to nanosecond dynamics ${ }^{100,101}$. The titrations were carried out by adding increasing amounts of a peptide stock solution to the NMR sample containing $0.138 \mathrm{mM}$ of protein and two-dimensional ${ }^{1} \mathrm{H}^{15} \mathrm{~N}-\mathrm{HSQC}$ spectra ${ }^{102}$ were recorded after thorough mixing of the Psb28-CP47 carboxyterminal peptide solution. Spectra were processed with NMRPipe ${ }^{92}$ and Psb28 ligand affinity calculations based on two-dimensional lineshape analysis were carried out using the TITAN software package ${ }^{103}$.

\section{Synthetic Peptide}

531 The carboxy-terminal peptide from residues 480-499 of CP47, which comprises the sequence SGIDPELSPEQVEWGFYQKV and includes an acetylated amino-terminus, was purchased from JPT Peptide Technologies GmbH, Germany. Peptide stock solutions of at least $6.07 \mathrm{mM}$

534 for titration experiments were prepared by dissolving the peptide in $20 \mathrm{mM}$ Tris/ $\mathrm{HCl} \mathrm{pH} 8$.

\section{Removal of the PSII oxygen evolving cluster}

536 PSII without functional oxygen evolving cluster (OEC) was prepared by isolating PSII as 537 described by Grasse et al. ${ }^{40}$, followed by removal of the extrinsic subunits according to Shen and Inoue ${ }^{104}$, with modifications. PSII was applied to a size exclusion column (Superdex 75 
10/300 GL, GE Healthcare) pre-equilibrated in $\mathrm{CaCl}_{2}$ buffer $(10 \mathrm{mM} \mathrm{MgCl} 2,20 \mathrm{mM}$ MES-

$\left.540 \mathrm{NaOH}, \mathrm{pH} 6.5,1 \mathrm{M} \mathrm{CaCl}_{2}, 0.03 \%(\mathrm{w} / \mathrm{v}) \mathrm{DDM}\right)$. PSII particles lacking the extrinsic subunits 541 and the $\mathrm{Mn}_{4} \mathrm{CaO}_{5}$ cluster, which were eluted in the void volume, were collected and the buffer 542 was exchanged to activity buffer $(100 \mathrm{mM} \mathrm{KCl}, 20 \mathrm{mM} \mathrm{MES}-\mathrm{KOH}, \mathrm{pH} 6.5,10 \mathrm{mM} \mathrm{MgCl}$, $54310 \mathrm{mM} \mathrm{CaCl}_{2}$ and $0.03 \%$ (w/v) DDM) using a spin concentrator (Amicon, Ultra - 15, 100000 544 NMWL).

\section{Detection of singlet oxygen by the room temperature EPR spectroscopy}

546 Singlet oxygen was trapped using the water-soluble spin-probe 2,2,6,6-tetramethyl-4547 piperidone (TEMPD) hydrochloride ${ }^{105}$ and measured with ESR300 (Bruker Biospin, 548 Rheinstetten, Germany). Samples (30 $\mu \mathrm{g} \mathrm{chl} \mathrm{ml-1)} \mathrm{were} \mathrm{illuminated} \mathrm{for} 1 \mathrm{~min}$ with red light 549 (RG 630) at $450 \mu \mathrm{mol}$ quanta $\mathrm{m}^{-2} \mathrm{~s}^{-1}$ in $0.5 \mathrm{M}$ mannitol, $10 \mathrm{mM} \mathrm{CaCl}_{2}, 10 \mathrm{mM} \mathrm{MgCl}_{2}, 20 \mathrm{mM}$ 550 MES at $\mathrm{pH}$ 6.5. Spectra were recorded using a flat cell containing $200 \mu \mathrm{l}$ sample. The 551 microwave power was $9.77 \mathrm{GHz}$ and $14.07 \mathrm{~mW}$ with a modulation frequency of $86 \mathrm{kHz}$ and 552 amplitude of $1.0 \mathrm{G}$. Each spectrum is an average of 8 scans, each with a sweep time of $10.5 \mathrm{~s}$.

\section{Cryo-electron microscopy}

554 For cryo-EM sample preparation, $4.5 \mu 1$ of purified protein complexes were applied to glow 555 discharged Quantifoil 2/1 grids, blotted for $3.5 \mathrm{~s}$ with force 4 in a Vitrobot Mark III (Thermo 556 Fisher) at $100 \%$ humidity and $4^{\circ} \mathrm{C}$, then plunge frozen in liquid ethane, cooled by liquid 557 nitrogen. Cryo-EM data was acquired with a FEI Titan Krios transmission electron microscope 558 using the SerialEM software ${ }^{106}$. Movie frames were recorded at a nominal magnification of $55922,500 x$ using a K3 direct electron detector (Gatan), The total electron dose of $\sim 55$ electrons 560 per $\AA 2$ was distributed over 30 frames at a calibrated physical pixel size of $1.09 \AA$. Micrographs 561 were recorded in a defocus range of -0.5 to $-3.0 \mu \mathrm{m}$.

\section{Image processing, classification and refinement}

563 Cryo-EM micrographs were processed on the fly using the Focus software package ${ }^{107}$ if they 564 passed the selection criteria (iciness $<1.05$, drift $0.4 \AA<\mathrm{x}<70 \AA$, defocus $0.5 \mathrm{um}<\mathrm{x}<5.5$ 565 um, estimated CTF resolution $<6 \AA$ ). Micrograph frames were aligned using MotionCor $2^{108}$ and the contrast transfer function (CTF) for aligned frames was determined using $\mathrm{Gctf}^{109}$. Using

567 Gautomatch (http://www.mrc-lmb.cam.ac.uk/kzhang/) 693,297 particles were picked template568 free on 824 acquired micrographs. Particles were extracted with a pixel box size of 260 using 569 RELION $3.1^{110}$ and imported into Cryosparc 2.3 ${ }^{111}$. After reference-free 2D classification, 570675,123 particles were used for ab initio construction of initial models and subjected to multiple 571 rounds of $3 \mathrm{D}$ classification to obtain models with and without Psb28 density. Non-uniform 
572 refinement in Cryosparc resulted in models with an estimated resolution of $\sim 3.2 \AA$. Particles

573 belonging to 3D classes with and without Psb28 (150,090 and 166,411 particles, respectively)

574 were reextracted in RELION with a pixel box size of 256 and subjected to several rounds of

575 CTF-refinement (estimation of anisotropic magnification, fit of per-micrograph defocus and

576 astigmatism and beam tilt estimation) and Bayesian polishing ${ }^{12}$. Both classes were refined

577 using the previously generated starting models. 3D classification without further alignment

578 using a mask around the Psb27 region separated particles in the Psb28-containing class into

579 distinct classes with and without Psb27 (57,862 and 91,473 particles, respectively). Final

580 refinement of each of the three classes (with Psb27 and Psb28 (PSII-I), with Psb28 but without

581 Psb27 (PSII-I'), and without Psb27 and Psb28 (PSII-M)) resulted in models with global

582 resolutions of $2.94 \AA, 2.76 \AA$ and $2.82 \AA$, respectively (Gold standard FSC analysis of two

583 independent half-sets at the 0.143 cutoff). Local-resolution and 3D-FSC plots (Extended Data

584 Fig. 2) were calculated using RELION and the "Remote 3DFSC Processing Server" web

585 interface $^{113}$, respectively.

\section{Atomic model construction}

587 The 3.6 Å resolution X-ray structure of monomeric PSII from T. elongatus with PDB-ID 3KZI ${ }^{44}$ 588 was used as initial structural model that was docked as rigid body using Chimera ${ }^{114}$ into the 589 obtained cryo EM densities for PSII-M and PSII-I. The cofactors that had no corresponding 590 density were removed. The subunit PsbJ was also removed, as it was deleted in the experimental 591 design. By highlighting the still unoccupied parts of the PSII-I density map, we identified 592 densities that lead to the structures of Psb27, Psb28, and Psb34.

593 The $2.4 \AA$ resolution X-ray structures of isolated Psb28 from T. elongatus with PDB-ID $5943 \mathrm{ZPN}^{115}$ and the $1.6 \AA$ resolution X-ray structure of isolated Psb27 from T. elongatus with 595 PDB-ID $2 \mathrm{Y}^{6} \mathrm{X}^{60}$ were docked as rigid bodies into the unoccupied densities. The $1.6 \AA$ 596 resolution X-ray structure of CyanoQ from T. elongatus with PDB-ID 3ZSU ${ }^{116}$ does not fit into 597 the density and was therefore not modeled.

598 As there was no experimentally resolved structural model of Psb34 available, we first used the 599 sequence with UniProt-ID Q8DMP8 to predict structures using the webserver SWISS Model ${ }^{117}$ 600 and LOMETS ${ }^{118}$. We also predicted the secondary structure through the meta server 601 Bioinformatics Toolkit ${ }^{119}$ and CCTOP ${ }^{120}$. The results of the secondary structure prediction are 602 summarized in Table S4. Combining these predictions together with the unassigned cryo-EM 603 density, we used COOT ${ }^{121}$ to build an initial model of Psb34 that has one $\alpha$-helix from amino 604 acid number 28 to 55 . 
606 The initial model of the complex described above was refined in real space against the cryo607 EM density of PSII-I, and structural clashes were removed using molecular dynamics flexible 608 fitting (MDFF) ${ }^{122}$. MDFF simulations were prepared in VMD 1.9.4a35 $5^{123}$ using QwikMD ${ }^{124}$ 609 and the MDFF plugin. The simulations were carried out with NAMD $2.13^{125}$ employing the 610 CHARMM36 force field. Secondary structure, cis peptide and chirality restraints where 611 employed during 800 steps of minimization followed by a 40 ps MDFF simulation at $300 \mathrm{~K}$. 612 Due to the employed restraints, only conformational changes of side chains and subunit 613 movements compared to the initial structure are identified during the initial MDFF run. We checked the fit to density of the structure by calculating cross-correlation values of the backbone atoms. For PSII-I, we identified residues 217 to 269 from PsbA and residues 467 to 499 from PsbB and PsbZ as main regions where the structural model was not yet in accordance with the density after the initial MDFF run. For these three regions, we employed an iterative combination of MDFF with Rosetta ${ }^{126,127}$. Here, we used the optimized strategy as described for model construction of the $26 \mathrm{~S}$ proteasome ${ }^{128,129}$.

To obtain an atomic model that fit the PSII-M density, we used the initial model based on 3KZI described above, but without PsbJ, Psb27, Psb28, and Psb34. After the initial MDFF run, the cross-correlation check did not reveal any regions with significant deviation between model and density. Therefore, no further refinement was necessary. This fast convergence reflects that there are no crucial differences between the PSII-M model and the X-ray structure 3KZI.

To obtain the atomic model that fit the PSII-I' density, we used the final PSII-I model without Psb27 for MDFF. After the initial MDFF run, the cross-correlation check did not reveal any regions with significant deviation between model and density. This fast convergence reflects that there are no crucial differences between the PSII-I and PSII-I' models, except for the presence of the Psb27 subunit.

Last, the PSII-M, PSII-I, and PSII-I' models were used to initiate one final round of real-space refinement in Phenix ${ }^{130}$.

\section{Acknowledgements}

634 We thank C. König, M. Völkel, and R. Oworah-Nkruma for excellent technical assistance, 635 Kristin Becker for cloning of the pIVEXPsb28His plasmid, Bibi Erjavec for preparation of the 636 scheme in Fig. 1 and Nicholas Cox for helpful discussion. J.M.S. is grateful to E. Conti for 637 scientific independence and great mentorship and to J. M. Plitzko and W. Baumeister for access to the cryo-EM infrastructure and early career support. M.M.N. is grateful to his mentor M. 
639 Rögner for generous support. Funding: Financial support was provided by the Max Planck 640 Society, the Helmholtz Zentrum München, the DFG research unit FOR2092 (EN 1194/1-1 to 641 B.D.E., NO 836/3-2 to M.M.N.), the DFG priority program 2002 (NO 836/4-1 to M.M.N.), the 642 grant NIH P41-GM104601 (to E.T.) and an Emmy-Noether fellowship (SCHU 3364/1-1 to 643 J.M.S). A.K.-L. was supported by the LabEx Saclay Plant Sciences-SPS (grant number ANR644 10-LABX-0040-SPS) and the French Infrastructure for Integrated Structural Biology (FRISBI; 645 grant number ANR-10-INSB-05). R.S. gratefully acknowledges support from the DFG (INST 646 213/757-1 FUGG and INST 213/843-1 FUGG). Author contributions: B.D.E., T.R., J.M.S. 647 and M.M.N. conceived the research, prepared the figures, and wrote the manuscript with the 648 contributions of all other authors. M.M.N. coordinated the activities. Preparation of mutants, 649 PSII isolation and biochemical analysis were performed by J.Z., M.M, P.L. and M.M.N. Mass 650 spectrometry analysis was done by J.M.-C. and J.D.L. J.M.S., S.B. and B.D.E. performed the 651 cryo-EM analysis and T.R. built the structural model with the help of S.K.S., A.C. and E.T. 652 Fluorescence spectroscopy was carried out by J.Z. and M.M.N. EPR experiments were 653 conducted by A.K.-L. NMR experiments were conducted and analyzed by O.A. and R.S.. All 654 authors approved the final version of the manuscript. Competing interests: The authors declare 655 no competing interests. Data availability: The cryo-EM density maps will be deposited in the 656 Electron Microscopy Data Bank, the atomic models of the cryo-EM structures in the worldwide 657 Protein Data Bank (wwPDB) and the NMR assignments for Psb28 in the Biological Magnetic 658 Resonance Bank (BMRB), respectively. 


\section{References}

6601 Hohmann-Marriott, M. F. \& Blankenship, R. E. Evolution of photosynthesis. Annual 661 review of plant biology 62, 515-548, doi:10.1146/annurev-arplant-042110-103811 662 663

2 Sanchez-Baracaldo, P. \& Cardona, T. On the origin of oxygenic photosynthesis and Cyanobacteria. New Phytol 225, 1440-1446, doi:10.1111/nph.16249 (2020).

3 Vinyard, D. J., Ananyev, G. M. \& Dismukes, G. C. Photosystem II: the reaction center of oxygenic photosynthesis. Annual review of biochemistry 82, 577-606, doi:10.1146/annurev-biochem-070511-100425 (2013).

4 Boekema, E. J. et al. Supramolecular structure of the photosystem II complex from green plants and cyanobacteria. Proc Natl Acad Sci U S A 92, 175-179, doi:10.1073/pnas.92.1.175 (1995).

5 Cox, N., Pantazis, D. A. \& Lubitz, W. Current Understanding of the Mechanism of Water Oxidation in Photosystem II and Its Relation to XFEL Data. Annual review of biochemistry 89, 795-820, doi:10.1146/annurev-biochem-011520-104801 (2020).

6 Shen, J. R. The Structure of Photosystem II and the Mechanism of Water Oxidation in Photosynthesis. Annual review of plant biology 66, 23-48, doi:10.1146/annurev-arplant050312-120129 (2015).

7 Yano, J. et al. Light-dependent production of dioxygen in photosynthesis. Met Ions Life Sci 15, 13-43, doi:10.1007/978-3-319-12415-5_2 (2015).

8 Ferreira, K. N., Iverson, T. M., Maghlaoui, K., Barber, J. \& Iwata, S. Architecture of the photosynthetic oxygen-evolving center. Science 303, 1831-1838, doi:10.1126/science.1093087 (2004).

9 Cardona, T., Sedoud, A., Cox, N. \& Rutherford, A. W. Charge separation in photosystem II: a comparative and evolutionary overview. Biochim Biophys Acta 1817, 26-43, doi:10.1016/j.bbabio.2011.07.012 (2012).

10 Holzwarth, A. R. et al. Kinetics and mechanism of electron transfer in intact photosystem II and in the isolated reaction center: pheophytin is the primary electron acceptor. Proc Natl Acad Sci U S A 103, 6895-6900, doi:10.1073/pnas.0505371103 (2006).

11 Müh, F., Glöckner, C., Hellmich, J. \& Zouni, A. Light-induced quinone reduction in photosystem II. Biochim Biophys Acta 1817, 44-65, doi:10.1016/j.bbabio.2011.05.021 (2012).

12 Faller, P. et al. Rapid formation of the stable tyrosyl radical in photosystem II. Proc Natl Acad Sci U S A 98, 14368-14373, doi:10.1073/pnas.251382598 (2001).

13 Roose, J. L., Frankel, L. K., Mummadisetti, M. P. \& Bricker, T. M. The extrinsic proteins of photosystem II: update. Planta 243, 889-908, doi:10.1007/s00425-0152462-6 (2016).

14 Müh, F. \& Zouni, A. Structural basis of light-harvesting in the photosystem II core complex. Protein Sci 29, 1090-1119, doi:10.1002/pro.3841 (2020).

15 Shi, L. X., Hall, M., Funk, C. \& Schröder, W. P. Photosystem II, a growing complex: updates on newly discovered components and low molecular mass proteins. Biochim Biophys Acta 1817, 13-25, doi:10.1016/j.bbabio.2011.08.008 (2012).

16 Stewart, D. H. \& Brudvig, G. W. Cytochrome b559 of photosystem II. Biochim Biophys Acta 1367, 63-87, doi:10.1016/s0005-2728(98)00139-x (1998).

17 Cox, N. et al. Photosynthesis. Electronic structure of the oxygen-evolving complex in photosystem II prior to O-O bond formation. Science 345, 804-808, doi:10.1126/science.1254910 (2014).

18 Kern, J. et al. Structures of the intermediates of Kok's photosynthetic water oxidation clock. Nature 563, 421-425, doi:10.1038/s41586-018-0681-2 (2018). 
19 Kupitz, C. et al. Serial time-resolved crystallography of photosystem II using a femtosecond X-ray laser. Nature 513, 261-265, doi:10.1038/nature13453 (2014).

20 Suga, M. et al. An oxyl/oxo mechanism for oxygen-oxygen coupling in PSII revealed by an X-ray free-electron laser. Science 366, 334-338, doi:10.1126/science.aax6998 (2019).

21 Umena, Y., Kawakami, K., Shen, J. R. \& Kamiya, N. Crystal structure of oxygenevolving photosystem II at a resolution of 1.9 A. Nature 473, 55-60, doi:10.1038/nature09913 (2011).

Krieger-Liszkay, A., Fufezan, C. \& Trebst, A. Singlet oxygen production in

23 Vass, I. Molecular mechanisms of photodamage in the Photosystem II complex. photosystem II and related protection mechanism. Photosynth Res 98, 551-564, doi:10.1007/s11120-008-9349-3 (2008).

24 Biochim Biophys Acta 1817, 209-217, doi:10.1016/j.bbabio.2011.04.014 (2012). biogenesis in cyanobacteria. Biochim Biophys Acta 1857, 274-287, doi:10.1016/j.bbabio.2015.11.007 (2016).

25 Nickelsen, J. \& Rengstl, B. Photosystem II assembly: from cyanobacteria to plants. Annual review of plant biology 64, 609-635, doi:10.1146/annurev-arplant-050312120124 (2013).

26 Nixon, P. J., Michoux, F., Yu, J., Boehm, M. \& Komenda, J. Recent advances in understanding the assembly and repair of photosystem II. Ann Bot 106, 1-16, doi:10.1093/aob/mcq059 (2010).

27 Komenda, J. et al. Cleavage after residue Ala352 in the C-terminal extension is an early step in the maturation of the D1 subunit of Photosystem II in Synechocystis PCC 6803. Biochim Biophys Acta 1767, 829-837, doi:10.1016/j.bbabio.2007.01.005 (2007).

28 Komenda, J. et al. The cyanobacterial homologue of HCF136/YCF48 is a component of an early photosystem II assembly complex and is important for both the efficient assembly and repair of photosystem II in Synechocystis sp. PCC 6803. J Biol Chem 283, 22390-22399, doi:10.1074/jbc.M801917200 (2008).

29 Boehm, M. et al. Subunit composition of CP43-less photosystem II complexes of Synechocystis sp. PCC 6803: implications for the assembly and repair of photosystem II. Philos Trans R Soc Lond B Biol Sci 367, 3444-3454, doi:10.1098/rstb.2012.0066 (2012).

30 Dobáková, M., Sobotka, R., Tichy, M. \& Komenda, J. Psb28 protein is involved in the biogenesis of the photosystem II inner antenna CP47 (PsbB) in the cyanobacterium Synechocystis sp. PCC 6803. Plant Physiol 149, 1076-1086, doi:10.1104/pp.108.130039 (2009).

31 Komenda, J. et al. The Psb27 assembly factor binds to the CP43 complex of photosystem II in the cyanobacterium Synechocystis sp. PCC 6803. Plant Physiol 158, 476-486, doi:10.1104/pp.111.184184 (2012).

32 Mamedov, F., Nowaczyk, M. M., Thapper, A., Rögner, M. \& Styring, S. Functional characterization of monomeric photosystem II core preparations from Thermosynechococcus elongatus with or without the Psb27 protein. Biochemistry 46, 5542-5551, doi:10.1021/bi7000399 (2007).

33 Nowaczyk, M. M. et al. Psb27, a cyanobacterial lipoprotein, is involved in the repair cycle of photosystem II. Plant Cell 18, 3121-3131, doi:10.1105/tpc.106.042671 (2006).

34 Roose, J. L. \& Pakrasi, H. B. The Psb27 protein facilitates manganese cluster assembly in photosystem II. J Biol Chem 283, 4044-4050, doi:10.1074/jbc.M708960200 (2008).

35 Nowaczyk, M. M. et al. Deletion of $p s b J$ leads to accumulation of Psb27-Psb28 photosystem II complexes in Thermosynechococcus elongatus. Biochim Biophys Acta 1817, 1339-1345, doi:10.1016/j.bbabio.2012.02.017 (2012). 
36 Sakata, S., Mizusawa, N., Kubota-Kawai, H., Sakurai, I. \& Wada, H. Psb28 is involved in recovery of photosystem II at high temperature in Synechocystis sp. PCC 6803. Biochim Biophys Acta 1827, 50-59, doi:10.1016/j.bbabio.2012.10.004 (2013).

764

37 Weisz, D. A. et al. Mass spectrometry-based cross-linking study shows that the Psb28 protein binds to cytochrome b559 in Photosystem II. Proc Natl Acad Sci U S A 114, 2224-2229, doi:10.1073/pnas.1620360114 (2017).

38 Bečková, M. et al. Association of Psb28 and Psb27 Proteins with PSII-PSI Supercomplexes upon Exposure of Synechocystis sp. PCC 6803 to High Light. Mol Plant 10, 62-72, doi:10.1016/j.molp.2016.08.001 (2017).

39 Bentley, F. K., Luo, H., Dilbeck, P., Burnap, R. L. \& Eaton-Rye, J. J. Effects of inactivating psbM and psbT on photodamage and assembly of photosystem II in Synechocystis sp. PCC 6803. Biochemistry 47, 11637-11646, doi:10.1021/bi800804h (2008).

40 Grasse, N. et al. Role of novel dimeric Photosystem II (PSII)-Psb27 protein complex in PSII repair. J Biol Chem 286, 29548-29555, doi:10.1074/jbc.M111.238394 (2011).

41 Liu, H., Roose, J. L., Cameron, J. C. \& Pakrasi, H. B. A genetically tagged Psb27 protein allows purification of two consecutive photosystem II (PSII) assembly intermediates in Synechocystis 6803, a cyanobacterium. J Biol Chem 286, 24865-24871, doi:10.1074/jbc.M111.246231 (2011).

42 Weisz, D. A. et al. A novel chlorophyll protein complex in the repair cycle of photosystem II. Proc Natl Acad Sci $U$ S A 116, 21907-21913, doi:10.1073/pnas.1909644116 (2019).

43 Mabbitt, P. D., Wilbanks, S. M. \& Eaton-Rye, J. J. Structure and function of the hydrophilic Photosystem II assembly proteins: Psb27, Psb28 and Ycf48. Plant Physiol Biochem 81, 96-107, doi:10.1016/j.plaphy.2014.02.013 (2014).

44 Broser, M. et al. Crystal structure of monomeric photosystem II from Thermosynechococcus elongatus at 3.6-Å resolution. J Biol Chem 285, 26255-26262, doi:10.1074/jbc.M110.127589 (2010).

45 Komenda, J. \& Sobotka, R. Cyanobacterial high-light-inducible proteins--Protectors of chlorophyll-protein synthesis and assembly. Biochim Biophys Acta 1857, 288-295, doi:10.1016/j.bbabio.2015.08.011 (2016).

46 Mulo, P. et al. Mutagenesis of the D-E loop of photosystem II reaction centre protein D1. Function and assembly of photosystem II. Plant Mol Biol 33, 1059-1071, doi:10.1023/a:1005765305956 (1997).

47 Eaton-Rye, J. J. \& Govindjee. Electron transfer through the quinone acceptor complex of photosystem II in bicarbonate-depleted spinach thylakoid membranes as a function of actinic flash number and frequency. Biochimica et Biophysica Acta (BBA)Bioenergetics 935, 237-247 (1988).

48 Allen, J. F. \& Nield, J. Redox Tuning in Photosystem II. Trends Plant Sci 22, 97-99, doi:10.1016/j.tplants.2016.11.011 (2017).

49 Brinkert, K., De Causmaecker, S., Krieger-Liszkay, A., Fantuzzi, A. \& Rutherford, A. W. Bicarbonate-induced redox tuning in Photosystem II for regulation and protection. Proc Natl Acad Sci U S A 113, 12144-12149, doi:10.1073/pnas.1608862113 (2016).

50 Vass, I., Kirilovsky, D. \& Etienne, A. L. UV-B radiation-induced donor- and acceptorside modifications of photosystem II in the cyanobacterium Synechocystis sp. PCC 6803. Biochemistry 38, 12786-12794, doi:10.1021/bi991094w (1999).

51 Johnson, G. N., Rutherford, A. W. \& Krieger, A. A change in the midpoint potential of the quinone QA in photosystem II associated with photoactivation of oxygen evolution. Biochim Biophys Acta 1229, 202-207 (1995). 
52 Johnson, G. N., Boussac, A. \& Rutherford, A. W. The origin of 40-50 C

810

811

812

813

814

815

816

817

818

819

820

821

822

823

824

825

826

827

828

829

830

831

832

833

834

835

836

837

838

839

840

841

842

843

844

845

846

847

848

849

850

851

852

853

854

855

856

857

858 thermoluminescence bands in photosystem II. Biochim Biophys Acta 1184, 85-92 (1994).

53 Stowell, M. H. et al. Light-induced structural changes in photosynthetic reaction center: implications for mechanism of electron-proton transfer. Science 276, 812-816, doi:10.1126/science.276.5313.812 (1997).

54 Cormann, K. U., Möller, M. \& Nowaczyk, M. M. Critical Assessment of Protein CrossLinking and Molecular Docking: An Updated Model for the Interaction Between Photosystem II and Psb27. Front Plant Sci 7, 157, doi:10.3389/fpls.2016.00157 (2016).

55 Liu, H., Huang, R. Y., Chen, J., Gross, M. L. \& Pakrasi, H. B. Psb27, a transiently associated protein, binds to the chlorophyll binding protein CP43 in photosystem II assembly intermediates. Proc Natl Acad Sci $U$ S A 108, 18536-18541, doi:10.1073/pnas.1111597108 (2011).

56 Wei, X. et al. Structure of spinach photosystem II-LHCII supercomplex at 3.2 A resolution. Nature 534, 69-74, doi:10.1038/nature18020 (2016).

57 Cormann, K. U. et al. Structure of Psb27 in solution: implications for transient binding to photosystem II during biogenesis and repair. Biochemistry 48, 8768-8770, doi:10.1021/bi9012726 (2009).

58 Fagerlund, R. D. \& Eaton-Rye, J. J. The lipoproteins of cyanobacterial photosystem II. J Photochem Photobiol B 104, 191-203, doi:10.1016/j.jphotobiol.2011.01.022 (2011).

59 Liu, H. et al. Mass spectrometry-based footprinting reveals structural dynamics of loop E of the chlorophyll-binding protein CP43 during photosystem II assembly in the cyanobacterium Synechocystis 6803. J Biol Chem 288, 14212-14220, doi:10.1074/jbc.M113.467613 (2013).

60 Michoux, F. et al. Crystal structure of the Psb27 assembly factor at 1.6 A: implications for binding to Photosystem II. Photosynth Res 110, 169-175, doi:10.1007/s11120-0119712-7 (2012).

61 Weisz, D. A., Gross, M. L. \& Pakrasi, H. B. The Use of Advanced Mass Spectrometry to Dissect the Life-Cycle of Photosystem II. Front Plant Sci 7, 617, doi:10.3389/fpls.2016.00617 (2016).

62 Kettunen, R., Tyystjarvi, E. \& Aro, E. M. Degradation pattern of photosystem II reaction center protein $\mathrm{D} 1$ in intact leaves. The major photoinhibition-induced cleavage site in D1 polypeptide is located amino terminally of the DE loop. Plant Physiol 111, 1183-1190, doi:10.1104/pp.111.4.1183 (1996).

63 Mulo, P., Laakso, S., Maenpaa, P. \& Aro, E. M. Stepwise photoinhibition of photosystem II. Studies with Synechocystis species PCC 6803 mutants with a modified D-E loop of the reaction center polypeptide D1. Plant Physiol 117, 483-490, doi:10.1104/pp.117.2.483 (1998).

64 Wang, X. et al. Is bicarbonate in Photosystem II the equivalent of the glutamate ligand to the iron atom in bacterial reaction centers? Biochim Biophys Acta 1100, 1-8, doi:10.1016/0005-2728(92)90119-m (1992).

65 Cheap, H. et al. M234Glu is a component of the proton sponge in the reaction center from photosynthetic bacteria. Biochim Biophys Acta 1787, 1505-1515, doi:10.1016/j.bbabio.2009.07.004 (2009).

66 Bao, H. \& Burnap, R. L. Photoactivation: The Light-Driven Assembly of the Water Oxidation Complex of Photosystem II. Front Plant Sci 7, 578, doi:10.3389/fpls.2016.00578 (2016).

67 Becker, K., Cormann, K. U. \& Nowaczyk, M. M. Assembly of the water-oxidizing complex in photosystem II. $J$ Photochem Photobiol B 104, 204-211, doi:10.1016/j.jphotobiol.2011.02.005 (2011). 
68 Cheniae, G. \& Martin, I. Photoactivation of the manganese catalyst of O2 evolution. I-

860

861

862

863

864

865

866

867

868

869

870

871

872

873

874

875

876

877

878

879

880

881

882

883

884

885

886

887

888

889

890

891

892

893

894

895

896

897

898

899

900

901

902

903

904

905

906

907

908

Biochemical and kinetic aspects. Biochim Biophys Acta 253, 167-181, doi:10.1016/0005-2728(71)90242-8 (1971).

69 Dasgupta, J., Ananyev, G. M. \& Dismukes, G. C. Photoassembly of the WaterOxidizing Complex in Photosystem II. Coord Chem Rev 252, 347-360, doi:10.1016/j.ccr.2007.08.022 (2008).

70 Zhang, M. et al. Structural insights into the light-driven auto-assembly process of the water-oxidizing Mn4CaO5-cluster in photosystem II. Elife 6, doi:10.7554/eLife.26933 (2017).

71 Gisriel, C. J. et al. Cryo-EM Structure of Monomeric Photosystem II from Synechocystis sp. PCC 6803 Lacking the Water-Oxidation Complex. Joule (2020).

72 Kolling, D. R., Cox, N., Ananyev, G. M., Pace, R. J. \& Dismukes, G. C. What are the oxidation states of manganese required to catalyze photosynthetic water oxidation? Biophys J 103, 313-322, doi:10.1016/j.bpj.2012.05.031 (2012).

73 Zaltsman, L., Ananyev, G. M., Bruntrager, E. \& Dismukes, G. C. Quantitative kinetic model for photoassembly of the photosynthetic water oxidase from its inorganic constituents: requirements for manganese and calcium in the kinetically resolved steps. Biochemistry 36, 8914-8922, doi:10.1021/bi970187f (1997).

74 Nixon, P. J. \& Diner, B. A. Aspartate 170 of the photosystem II reaction center polypeptide D1 is involved in the assembly of the oxygen-evolving manganese cluster. Biochemistry 31, 942-948, doi:10.1021/bi00118a041 (1992).

75 Tyryshkin, A. M. et al. Spectroscopic evidence for $\mathrm{Ca} 2+$ involvement in the assembly of the $\mathrm{Mn} 4 \mathrm{Ca}$ cluster in the photosynthetic water-oxidizing complex. Biochemistry 45, 12876-12889, doi:10.1021/bi061495t (2006).

76 Campbell, K. A. et al. Dual-mode EPR detects the initial intermediate in photoassembly of the photosystem II Mn cluster: the influence of amino acid residue 170 of the D1 polypeptide on Mn coordination. Journal of the American Chemical Society 122, 37543761 (2000).

77 Cohen, R. O., Nixon, P. J. \& Diner, B. A. Participation of the C-terminal region of the D1-polypeptide in the first steps in the assembly of the Mn4Ca cluster of photosystem II. J Biol Chem 282, 7209-7218, doi:10.1074/jbc.M606255200 (2007).

78 Kuhl, H. et al. Towards structural determination of the water-splitting enzyme. Purification, crystallization, and preliminary crystallographic studies of photosystem II from a thermophilic cyanobacterium. $J$ Biol Chem 275, 20652-20659, doi:10.1074/jbc.M001321200 (2000).

79 Iwai, M., Katoh, H., Katayama, M. \& Ikeuchi, M. Improved genetic transformation of the thermophilic cyanobacterium, Thermosynechococcus elongatus BP-1. Plant Cell Physiol 45, 171-175, doi:10.1093/pcp/pch015 (2004).

80 Cormann, K. U., Bartsch, M., Rögner, M. \& Nowaczyk, M. M. Localization of the CyanoP binding site on photosystem II by surface plasmon resonance spectroscopy. Front Plant Sci 5, 595, doi:10.3389/fpls.2014.00595 (2014).

81 Studier, F. W. Protein production by auto-induction in high density shaking cultures. Protein Expr Purif 41, 207-234, doi:10.1016/j.pep.2005.01.016 (2005).

82 Neff, D. \& Dencher, N. A. Purification of multisubunit membrane protein complexes: isolation of chloroplast FoF1-ATP synthase, CFo and CF1 by blue native electrophoresis. Biochem Biophys Res Commun 259, 569-575, doi:10.1006/bbrc.1999.0820 (1999).

83 Schagger, H. \& von Jagow, G. Tricine-sodium dodecyl sulfate-polyacrylamide gel electrophoresis for the separation of proteins in the range from 1 to $100 \mathrm{kDa}$. Anal Biochem 166, 368-379, doi:10.1016/0003-2697(87)90587-2 (1987). 
84 Grzesiek, S. \& Bax, A. Improved 3D triple-resonance NMR techniques applied to a 31 kDa protein. Journal of Magnetic Resonance (1969) 96, 432-440 (1992). Schleucher, J., Sattler, M. \& Griesinger, C. Coherence selection by gradients without signal attenuation: application to the three-dimensional HNCO experiment. Angew Chem Int Ed Engl 32, 1489-1491 (1993).

87 Vuister, G. W. \& Bax, A. Quantitative J correlation: a new approach for measuring homonuclear three-bond $\mathrm{J}(\mathrm{HNH}$. alpha.) coupling constants in $15 \mathrm{~N}$-enriched proteins. J. Am. Chem. Soc. 115, $7772-7777$ (1993).

88 Kay, L. E., Xu, G.-Y., Singer, A. U., Muhandiram, D. R. \& Forman-Kay, J. D. A gradient-enhanced HCCH-TOCSY experiment for recording side-chain $1 \mathrm{H}$ and $13 \mathrm{C}$ correlations in $\mathrm{H} 2 \mathrm{O}$ samples for proteins. Journal of magnetic resonance. Series B 101, 333-337 (1993).

89 Sattler, M., Schwalbe, H. \& Griesinger, C. Stereospecific assignment of leucine methyl groups with carbon-13 in natural abundance or with random $13 \mathrm{C}$ labeling. $J$ Am Chem Soc 114, 1126-1127 (1992).

90 Davis, J. H. Refocusing revisited: an optimized, gradient-enhanced refocused HSQC and its applications in 2D and 3D NMR and in deuterium exchange experiments. $J$ Biomol NMR 5, 433-437, doi:10.1007/BF00182288 (1995).

91 Davis, A. L., Keeler, J., Laue, E. D. \& Moskau, D. Experiments for recording pureabsorption heteronuclear correlation spectra using pulsed field gradients. Journal of Magnetic Resonance 98, 207-216 (1992).

92 Delaglio, F. et al. NMRPipe: a multidimensional spectral processing system based on UNIX pipes. J Biomol NMR 6, 277-293, doi:10.1007/BF00197809 (1995).

93 Vranken, W. F. et al. The CCPN data model for NMR spectroscopy: development of a software pipeline. Proteins 59, 687-696, doi:10.1002/prot.20449 (2005).

94 Yamazaki, T., Lee, W., Arrowsmith, C. H., Muhandiram, D. R. \& Kay, L. E. A suite of triple resonance NMR experiments for the backbone assignment of $15 \mathrm{~N}, 13 \mathrm{C}, 2 \mathrm{H}$ labeled proteins with high sensitivity. J. Am. Chem. Soc. 116, 11655-11666 (1994).

95 Muhandiram, D., Xu, G. Y. \& Kay, L. E. An enhanced-sensitivity pure absorption gradient 4D 15 N, 13 C-edited NOESY experiment. Journal of Biomolecular NMR 3, 463-470 (1993).

96 Wittekind, M. \& Mueller, L. HNCACB, a high-sensitivity 3D NMR experiment to correlate amide-proton and nitrogen resonances with the alpha-and beta-carbon resonances in proteins. Journal of magnetic resonance. Series B 101, 201-205 (1993).

97 Clubb, R., Thanabal, V. \& Wagner, G. Constant time 3-dimensional triple resonance pulse scheme to intraresidue H-1 (N), N-15, C-13' chemical shifts in N-15, C-13 enriched proteins. J. Magn. Reson 97, 213-217 (1992).

98 Logan, T. M., Olejniczak, E. T., Xu, R. X. \& Fesik, S. W. A general method for assigning NMR spectra of denatured proteins using 3D HC(CO)NH-TOCSY triple resonance experiments. J Biomol NMR 3, 225-231, doi:10.1007/BF00178264 (1993).

99 Lyons, B. \& Montelione, G. An HCCNH triple-resonance experiment using carbon-13 isotropic mixing for correlating backbone amide and side-chain aliphatic resonances in isotopically enriched proteins. Journal of magnetic resonance. Series B (Print) 101, 206-209 (1993).

100 Barbato, G., Ikura, M., Kay, L. E., Pastor, R. W. \& Bax, A. Backbone dynamics of calmodulin studied by $15 \mathrm{~N}$ relaxation using inverse detected two-dimensional NMR spectroscopy: the central helix is flexible. Biochemistry 31, 5269-5278, doi:10.1021/bi00138a005 (1992). 
101 Ross, A., Czisch, M., Zinc, T. \& Holak, T. A. Improved spectral quality in measurements of heteronuclear NOE enhancement in proteins in water using pulsed field gradients. Journal of magnetic resonance. Series B (Print) 102, 314-316 (1993).

102 Mori, S., Abeygunawardana, C., Johnson, M. O. \& van Zijl, P. C. Improved sensitivity of HSQC spectra of exchanging protons at short interscan delays using a new fast HSQC (FHSQC) detection scheme that avoids water saturation. J Magn Reson B 108, 94-98, doi:10.1006/jmrb.1995.1109 (1995).

103 Waudby, C. A., Ramos, A., Cabrita, L. D. \& Christodoulou, J. Two-Dimensional NMR Lineshape Analysis. Sci Rep 6, 24826, doi:10.1038/srep24826 (2016).

104 Shen, J. R. \& Inoue, Y. Binding and functional properties of two new extrinsic components, cytochrome c-550 and a 12-kDa protein, in cyanobacterial photosystem II. Biochemistry 32, 1825-1832, doi:10.1021/bi00058a017 (1993).

105 Hideg, É. et al. Pure forms of the singlet oxygen sensors TEMP and TEMPD do not inhibit Photosystem II. Biochim Biophys Acta 1807, 1658-1661 (2011).

106 Mastronarde, D. N. Automated electron microscope tomography using robust prediction of specimen movements. Journal of structural biology 152, 36-51 (2005).

107 Biyani, N. et al. Focus: The interface between data collection and data processing in cryo-EM. J Struct Biol 198, 124-133, doi:10.1016/j.jsb.2017.03.007 (2017).

108 Zheng, S. Q. et al. MotionCor2: anisotropic correction of beam-induced motion for improved cryo-electron microscopy. Nat Methods 14, 331-332, doi:10.1038/nmeth.4193 (2017).

109 Zhang, K. Gctf: Real-time CTF determination and correction. J Struct Biol 193, 1-12, doi:10.1016/j.jsb.2015.11.003 (2016).

110 Scheres, S. H. A Bayesian view on cryo-EM structure determination. J Mol Biol 415, 406-418, doi:10.1016/j.jmb.2011.11.010 (2012).

111 Punjani, A., Rubinstein, J. L., Fleet, D. J. \& Brubaker, M. A. cryoSPARC: algorithms for rapid unsupervised cryo-EM structure determination. Nat Methods 14, 290-296, doi:10.1038/nmeth.4169 (2017).

112 Scheres, S. H. Beam-induced motion correction for sub-megadalton cryo-EM particles. Elife 3, e03665, doi:10.7554/eLife.03665 (2014).

113 Tan, Y. Z. et al. Addressing preferred specimen orientation in single-particle cryo-EM through tilting. Nat Methods 14, 793-796, doi:10.1038/nmeth.4347 (2017).

114 Pettersen, E. F. et al. UCSF Chimera--a visualization system for exploratory research and analysis. J Comput Chem 25, 1605-1612, doi:10.1002/jcc.20084 (2004).

115 Bialek, W. et al. Crystal structure of the Psb28 accessory factor of Thermosynechococcus elongatus photosystem II at 2.3 A. Photosynth Res 117, 375 383, doi:10.1007/s11120-013-9939-6 (2013).

116 Michoux, F. et al. Crystal structure of CyanoQ from the thermophilic cyanobacterium Thermosynechococcus elongatus and detection in isolated photosystem II complexes. Photosynth Res 122, 57-67, doi:10.1007/s11120-014-0010-z (2014).

117 Schwede, T., Kopp, J., Guex, N. \& Peitsch, M. C. SWISS-MODEL: An automated protein homology-modeling server. Nucleic Acids Res 31, 3381-3385, doi:10.1093/nar/gkg520 (2003).

118 Wu, S. \& Zhang, Y. LOMETS: a local meta-threading-server for protein structure prediction. Nucleic Acids Res 35, 3375-3382, doi:10.1093/nar/gkm251 (2007).

119 Zimmermann, L. et al. A Completely Reimplemented MPI Bioinformatics Toolkit with a New HHpred Server at its Core. $J$ Mol Biol 430, 2237-2243, doi:10.1016/j.jmb.2017.12.007 (2018).

120 Dobson, L., Remenyi, I. \& Tusnady, G. E. CCTOP: a Consensus Constrained TOPology prediction web server. Nucleic Acids Res 43, W408-412, doi:10.1093/nar/gkv451 (2015). 
121 Emsley, P., Lohkamp, B., Scott, W. G. \& Cowtan, K. Features and development of Coot. Acta Crystallogr D Biol Crystallogr 66, 486-501, doi:10.1107/S0907444910007493 (2010).

122 Trabuco, L. G., Villa, E., Schreiner, E., Harrison, C. B. \& Schulten, K. Molecular dynamics flexible fitting: a practical guide to combine cryo-electron microscopy and Xray crystallography. Methods 49, 174-180, doi:10.1016/j.ymeth.2009.04.005 (2009).

123 Humphrey, W., Dalke, A. \& Schulten, K. VMD: visual molecular dynamics. J Mol Graph 14, 33-38, doi:10.1016/0263-7855(96)00018-5 (1996).

124 Ribeiro, J. V. et al. QwikMD - Integrative Molecular Dynamics Toolkit for Novices and Experts. Sci Rep 6, 26536, doi:10.1038/srep26536 (2016).

125 Phillips, J. C. et al. Scalable molecular dynamics with NAMD. J Comput Chem 26, 1781-1802, doi:10.1002/jcc.20289 (2005).

126 Leaver-Fay, A. et al. ROSETTA3: an object-oriented software suite for the simulation and design of macromolecules. Methods Enzymol 487, 545-574, doi:10.1016/B978-012-381270-4.00019-6 (2011).

127 Lindert, S. \& McCammon, J. A. Improved cryoEM-Guided Iterative Molecular Dynamics--Rosetta Protein Structure Refinement Protocol for High Precision Protein Structure Prediction. J Chem Theory Comput 11, 1337-1346, doi:10.1021/ct500995d (2015).

128 Guo, Q. et al. In Situ Structure of Neuronal C9orf72 Poly-GA Aggregates Reveals Proteasome Recruitment. Cell 172, 696-705 e612, doi:10.1016/j.cell.2017.12.030 (2018).

129 Wehmer, M. et al. Structural insights into the functional cycle of the ATPase module of the 26S proteasome. Proc Natl Acad Sci $U$ S A 114, 1305-1310, doi:10.1073/pnas.1621129114 (2017).

130 Liebschner, D. et al. Macromolecular structure determination using X-rays, neutrons and electrons: recent developments in Phenix. Acta Crystallogr D Struct Biol 75, 861877, doi:10.1107/S2059798319011471 (2019). 


\section{Figures}

A

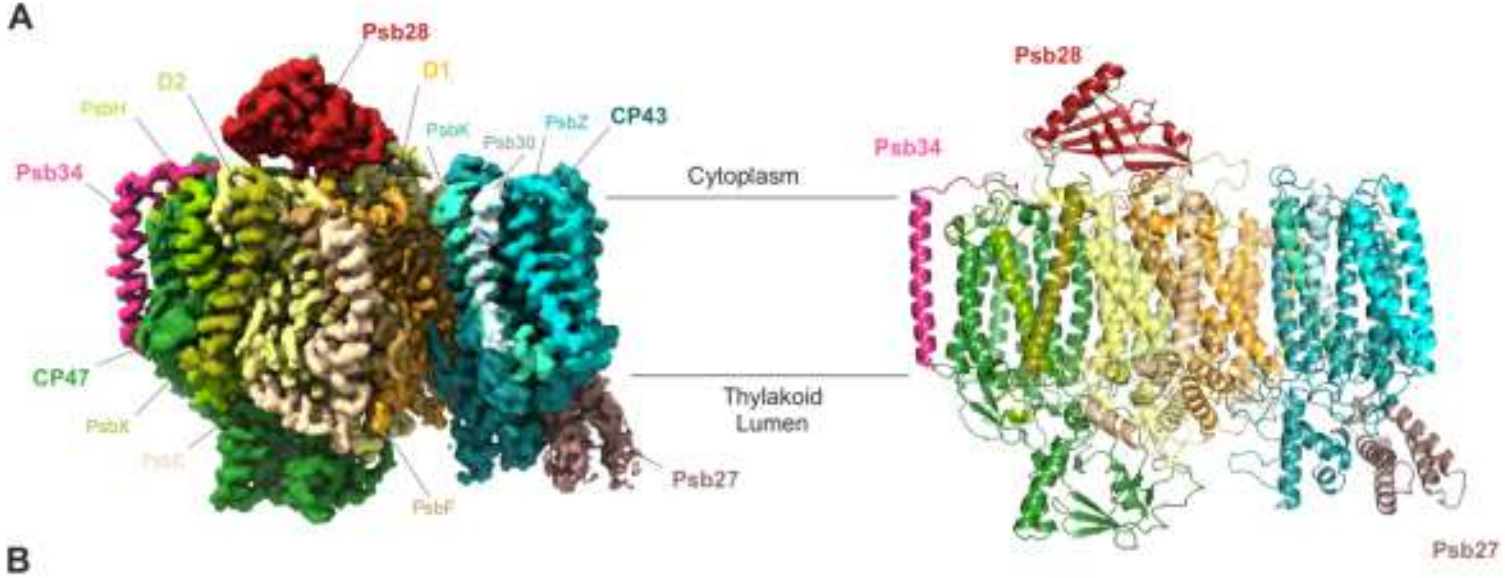

B
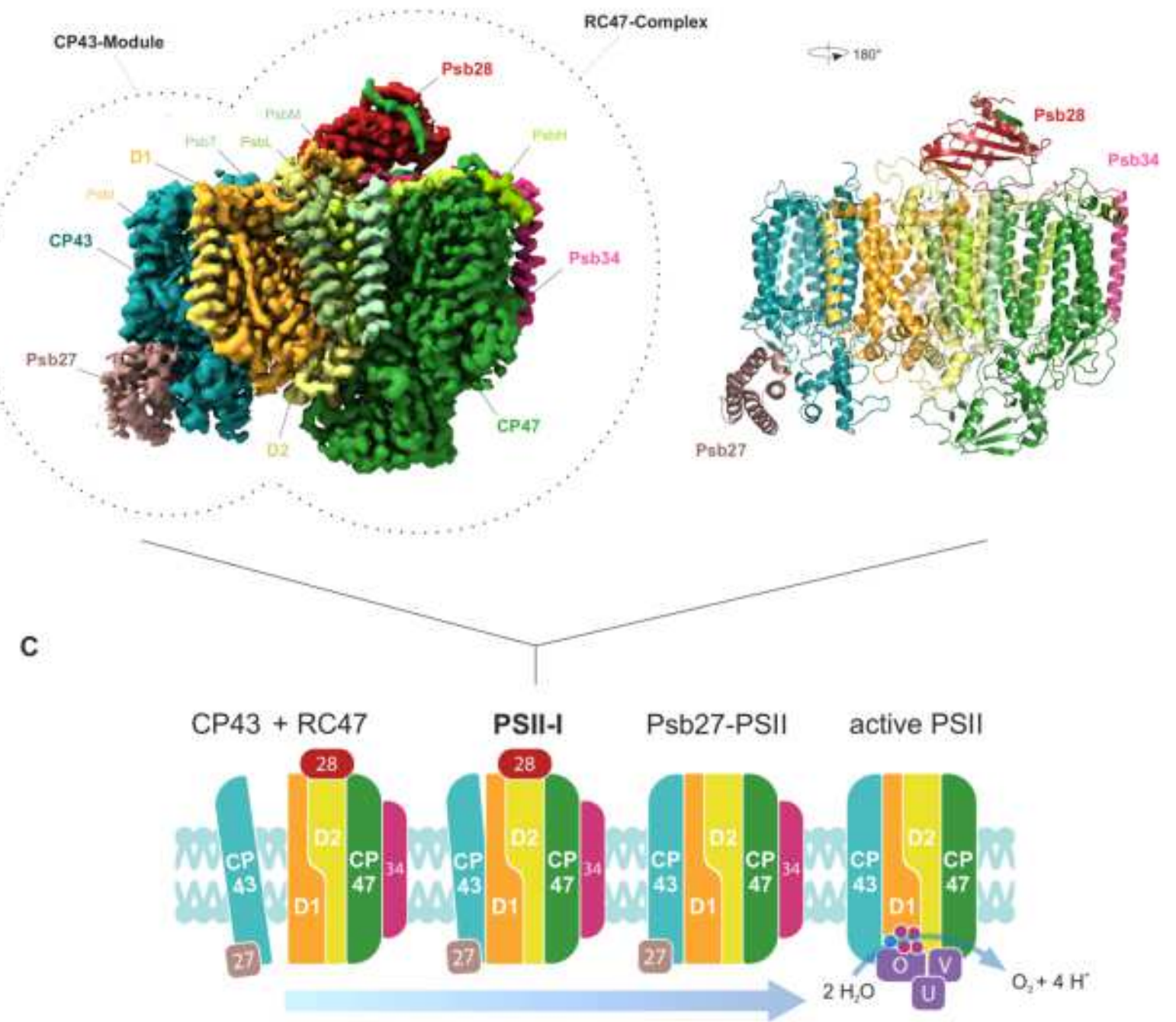

Figure 1

Cryo-EM map of a PSII assembly intermediate (PSII-I) from T. elongatus, segmented by subunit. (A) 15 PSII subunits and 3 assembly factors are colored and named (PSIl subunits: D1, D2, CP43, CP47, PsbE, PsbF, PsbH, Psbl, PsbK, PsbL, PsbM, PsbT, PsbX, PsbZ and Psb30; assembly factors: Psb27, Psb28 and 
ts10063, which we named Psb34) (front view). (B) Parts of PSIl that originate from the CP43 module (comprised of CP43, Psb27, PsbZ, Psb30 and PsbK) and the RC47 complex are indicated by dashed lines (back view). Schematic model of the PSII assembly process starting with the formation of PSII-I from the CP43 module and RC47. Small PSII subunits were omitted for simplicity.
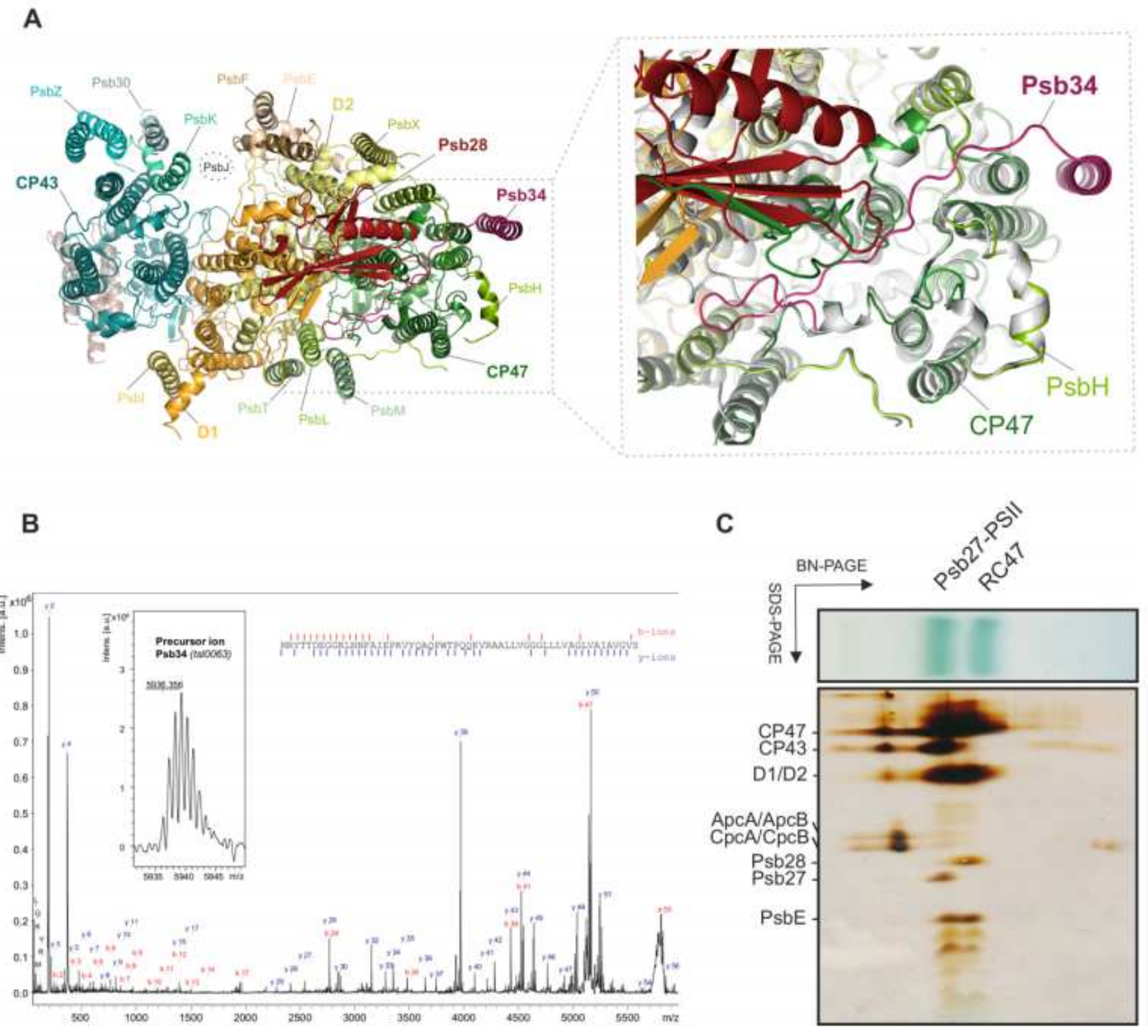

Figure 2

Psb34 binds to RC47 during attachment of the CP43 module. (A) Binding site of Psb34 at CP47, close to $\mathrm{PsbH}$ (top view), with extended binding of the Psb34 N-terminus along the cytoplasmic PSIl surface (dashed box). (B) MALDI-ToF analysis of PSII assembly intermediates. Mass spectrum of Psb34 (tsl0063) from the PSII complex (inset) and the fragment spectrum obtained for $\mathrm{m} / \mathrm{z} 5936.356$ with annotated $b$ - and $y$-ion series matching the Psb34 sequence. Observed fragmentation sites are indicated 
by dashes in the sequence. Mascot score: 171. (C) Subunit composition of Psb34-PSIl assembly intermediates analyzed by 2D-PAGE.

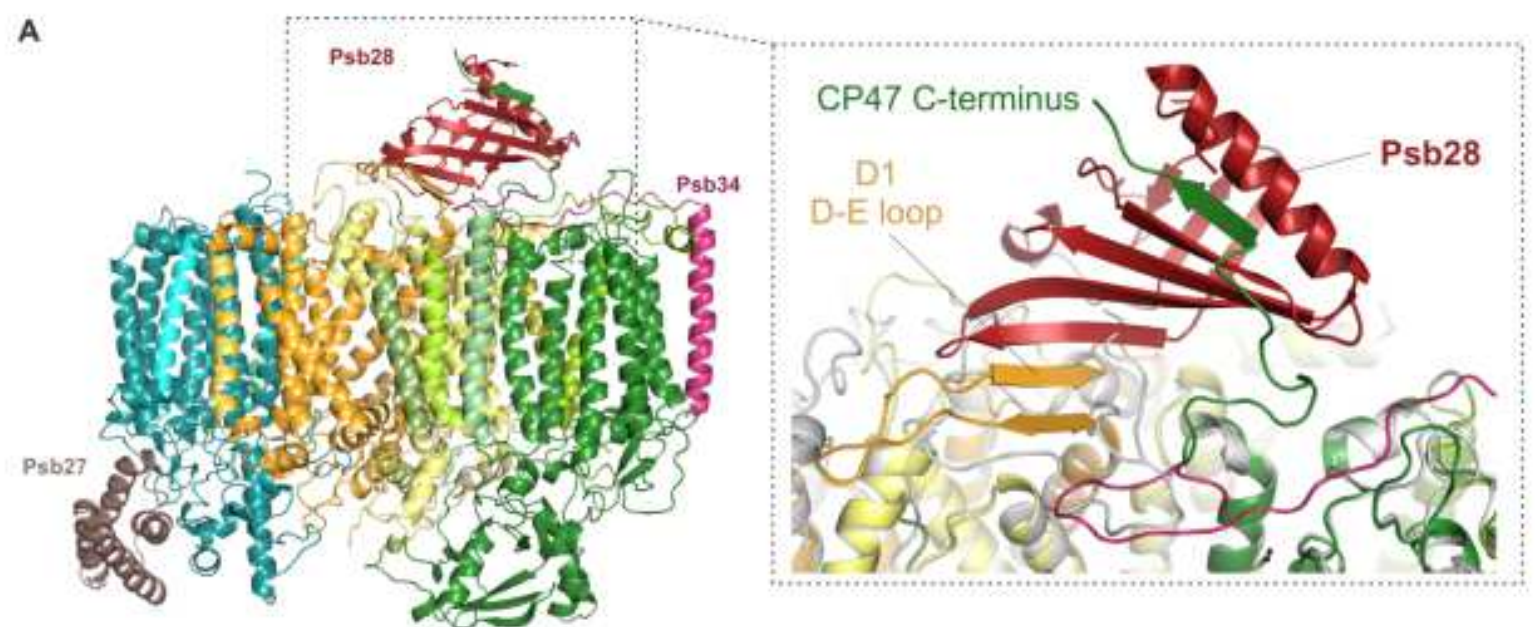

B

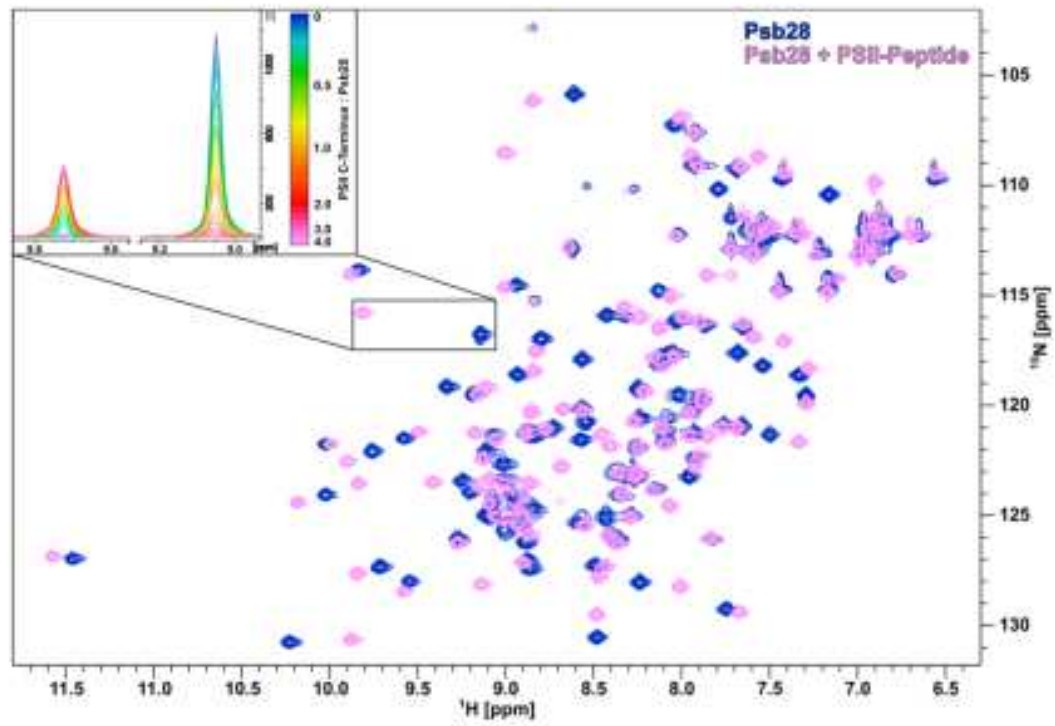

C

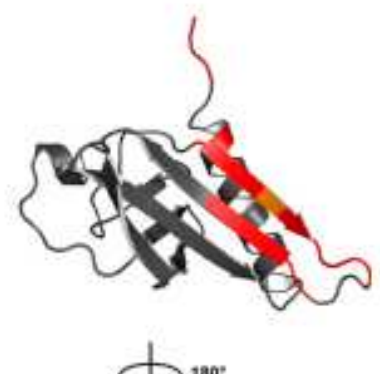

D

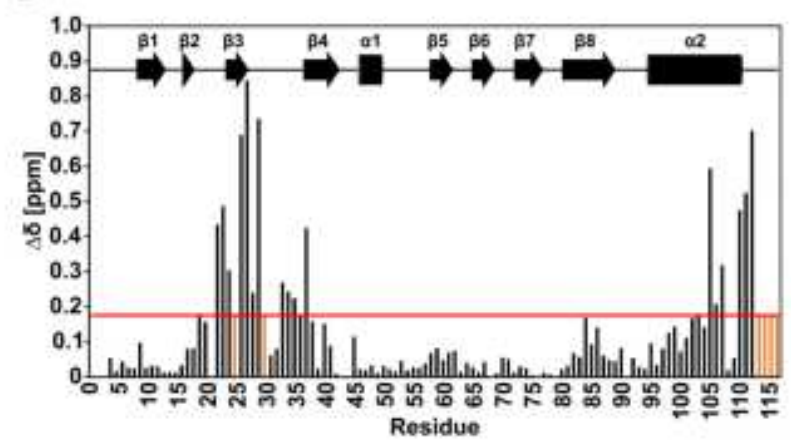

E

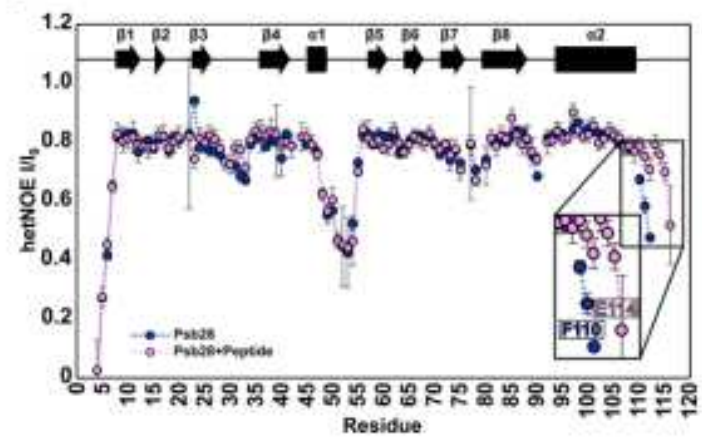

Figure 3

: The role of the CP47 C-terminus in binding of Psb28. (A). Binding of Psb28 at the cytoplasmic/stromal PSIl surface (side view, colors correspond to Fig. 1) and continuation of the central Psb28 beta-sheet by the CP47 C-terminus and the D-E loop of D1 (dashed box). For comparison, mature monomeric PSII (PDB- 
ID 3KZI) is shown in gray. (B) Superimposed 2D 1 H15N-HSQC spectra of free Psb28 (blue) and Psb28 bound to the Cterminal peptide of CP47 (magenta). Upper left inset: representation of slow exchange behavior for the proton amide resonance of T24, ranging from $126.9 \mathrm{ppm}$ to $128.6 \mathrm{ppm}$ in the $15 \mathrm{~N}$ dimension. (C) CSPs of more than one SD projected onto the model representation of Psb28. (D) Weighted $1 \mathrm{H} / 15 \mathrm{~N}$ chemical shift perturbations observed 11 for Psb28 upon binding to the CP47 peptide. Red line indicates one standard deviation (SD), residues that yield resonances only in the complex form are indicated in orange. (E) Backbone 15N $\{1 \mathrm{H}\}$-heteronuclear NOE of free Psb28 (blue) and Psb28 bound to the $\mathrm{C}$-terminal region of the $\mathrm{CP} 47$ peptide (magenta). Smaller $\mathrm{I} / \mathrm{IO}$ ratios correspond to regions that exhibit dynamics on the pico- to nanosecond timescale.

A

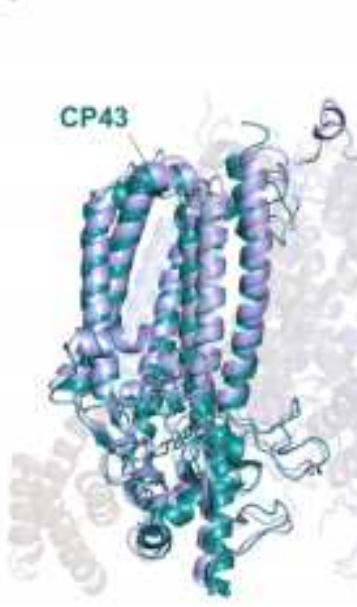

C

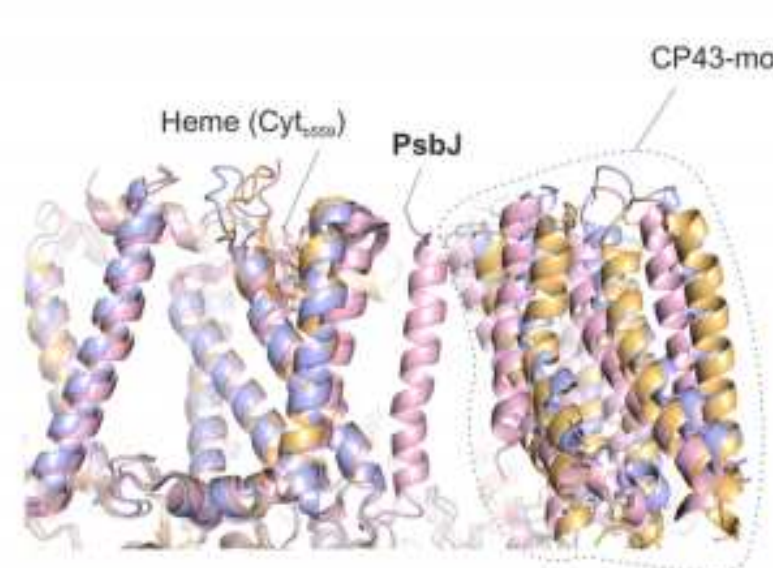

B

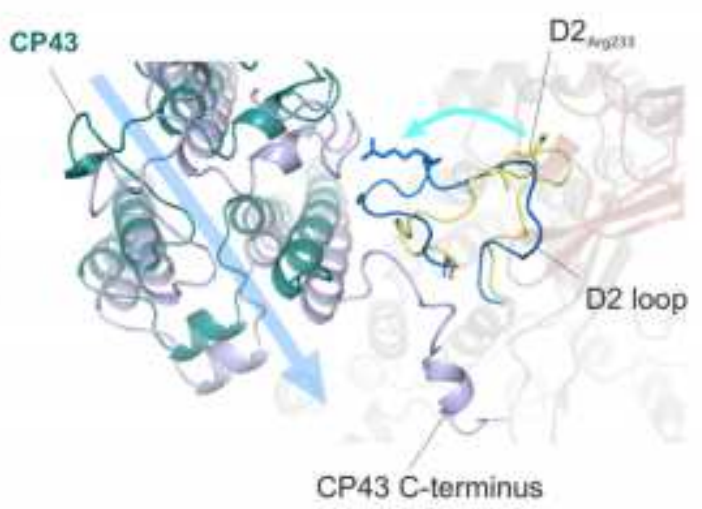

D

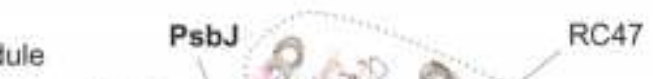

E

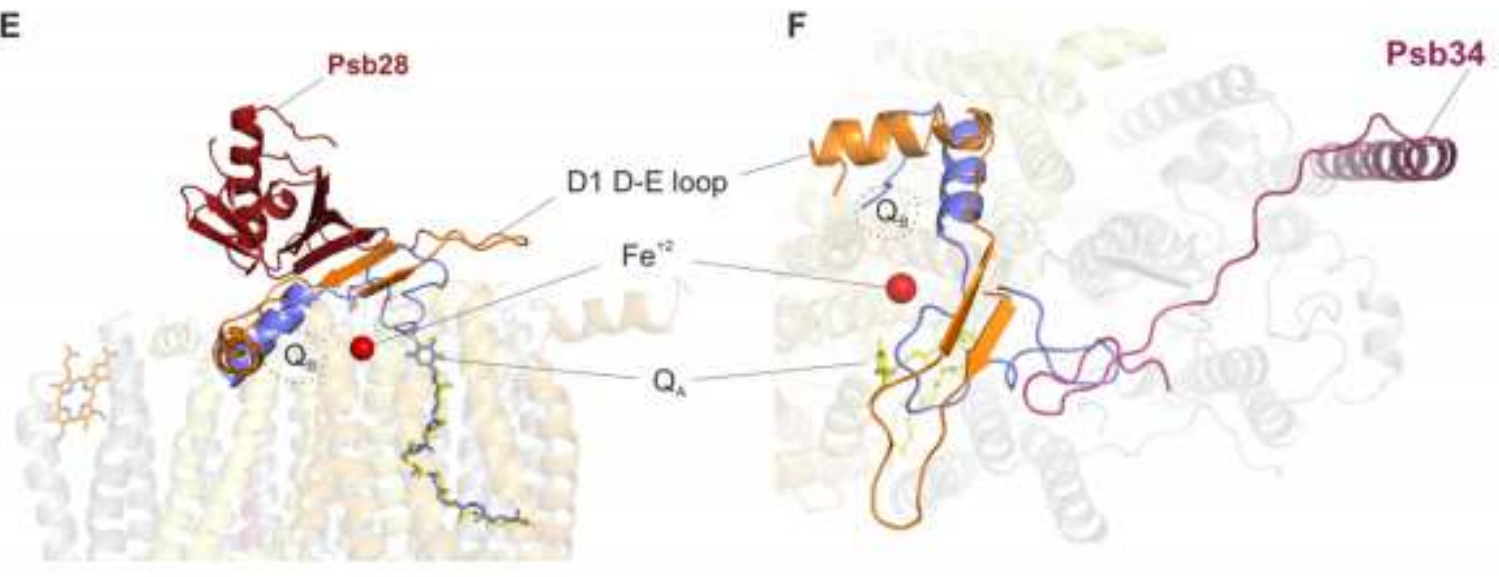




\section{Figure 4}

Structural changes of the D1 and D2 D-E loops induced by binding of Psb28 and Psb34. (A) Side view of the CP43 antenna protein in PSII-I (teal) and the PSII-M control (light blue). (B) Structural changes between PSII-I and the PSII-M control in the cytoplasmic D2 D-E loop (yellow: PSIII, blue: PSII-M) and attachment of CP43 (teal: PSII-I, light blue: PSII-M control) (top view). Details of the structural changes in the D2 loop are shown in Fig. S5A and B. (C) Side view and (D) top view of the PSII-I structure (orange) compared to the PSIIM control (light blue) and mature monomeric PSII (light red, PDB-ID 3KZI). (E) Side view and $(F)$ top view of the Psb28-induced structural changes in the D1 D-E loop (orange) and perturbation of the QB binding site compared to PSII-M (light blue), which lacks the assembly factors. QA is shown in yellow (PSII-I) or light blue (PSII-M), respectively. See Fig. S5C-H for enlarged views of the QA and QB binding site and the adjacent nonheme iron. 
A

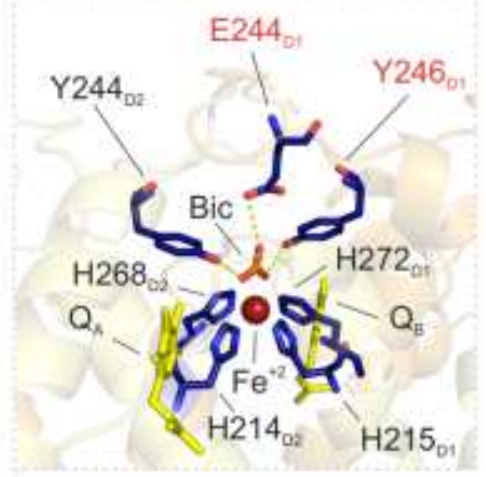

C

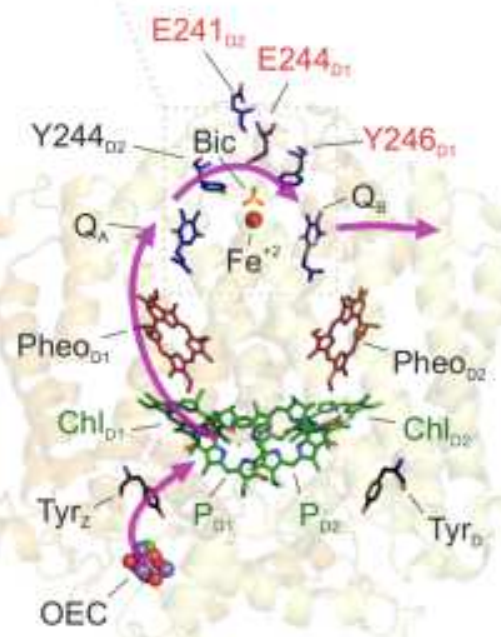

$E$

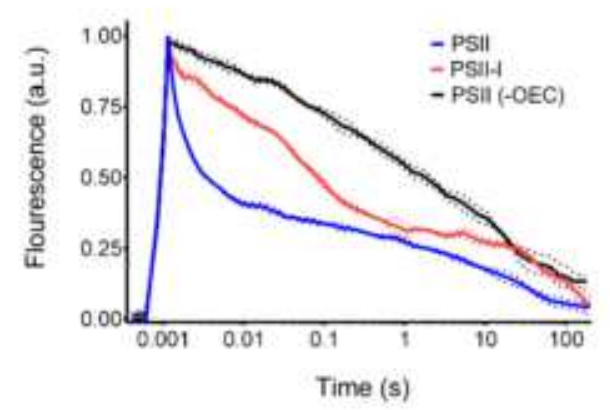

B

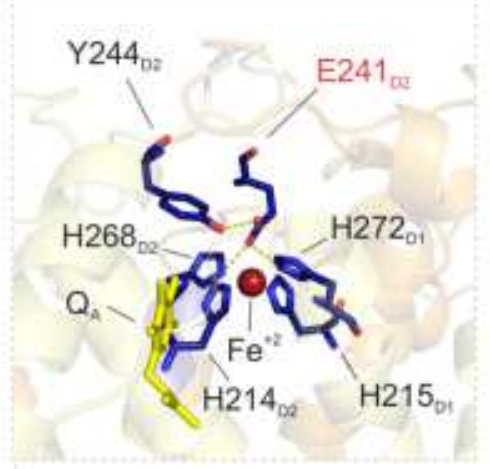

D
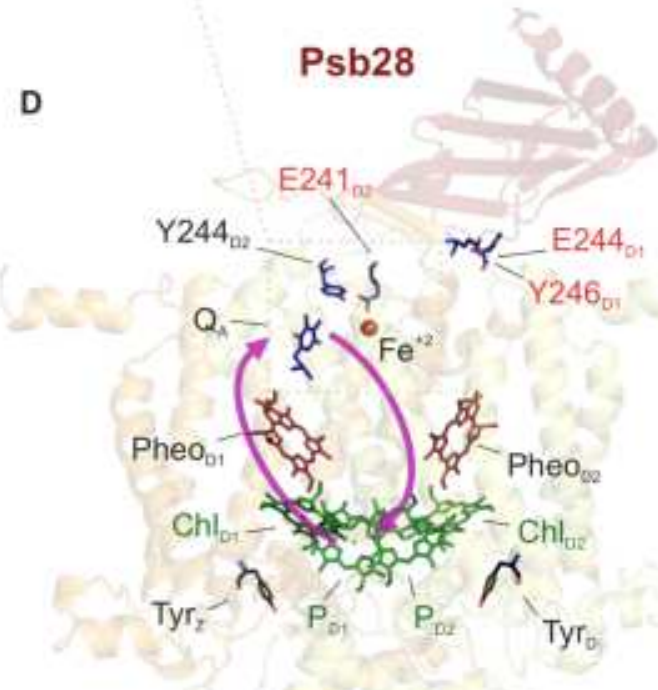

$\mathrm{F}$

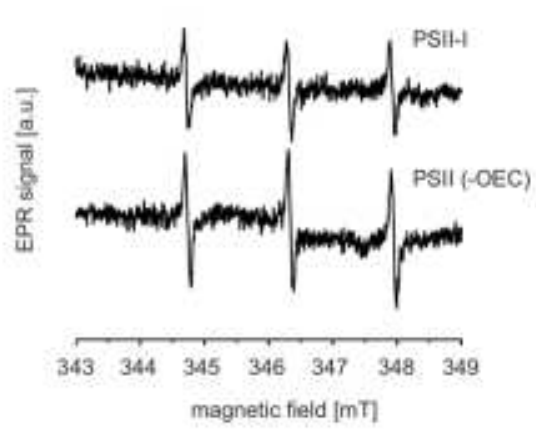

\section{Figure 5}

Binding of Psb28 displaces bicarbonate as a ligand of the non-heme iron and protects PSII from damage. (A) The electron transfer from $P Q A$ to $P Q B$ is coordinated by the non-heme iron (Fe2+), with the binding of bicarbonate (Bic) serving as a regulatory mechanism49 in mature PSII (PDB-ID 3WU2). (B) Binding of Psb28 to the PSII-I assembly intermediate induces a conformational change in the cytoplasmic D2 D-E loop, where the side chain of Glu241 replaces bicarbonate as a ligand of the non-heme iron. The 
respective fits of the non-heme iron binding sites are shown in Fig. S5E and F. A similar coordination is also found in non-oxygenic bacterial reaction centers53 (Fig. S6C). (C) Electron transfer (purple arrows) in mature PSII. Light-induced charge separation at the reaction center chlorophylls (PD1, PD2, ChID1, ChID2) leads to electron transfer via pheophytin (PheoD1) and plastoquinone A (QA) towards QB. The electron gap at the reaction center is filled by the oxygen evolving complex (OEC). (D) Reoxidation of QA- by direct and safe charge recombination is favored in the PSII assembly intermediate, as indicated by the purple arrows. (E) Flash-induced fluorescence decay of PSII. Blue lines represent active PSII, black and red represent PSII without functional OEC and PSII-I respectively. Dotted corridors depict SD $(n=3)$. (F) The protective role of Psb28 binding was further confirmed by EPR spectroscopy using the spin probe TEMPD, which is specific for 102 , the major reactive oxygen species in PSIl generated by triplet chlorophyll (3 P). Inactivated PSIl without functional Mn4CaO5 cluster (-OEC) was used as control.

A

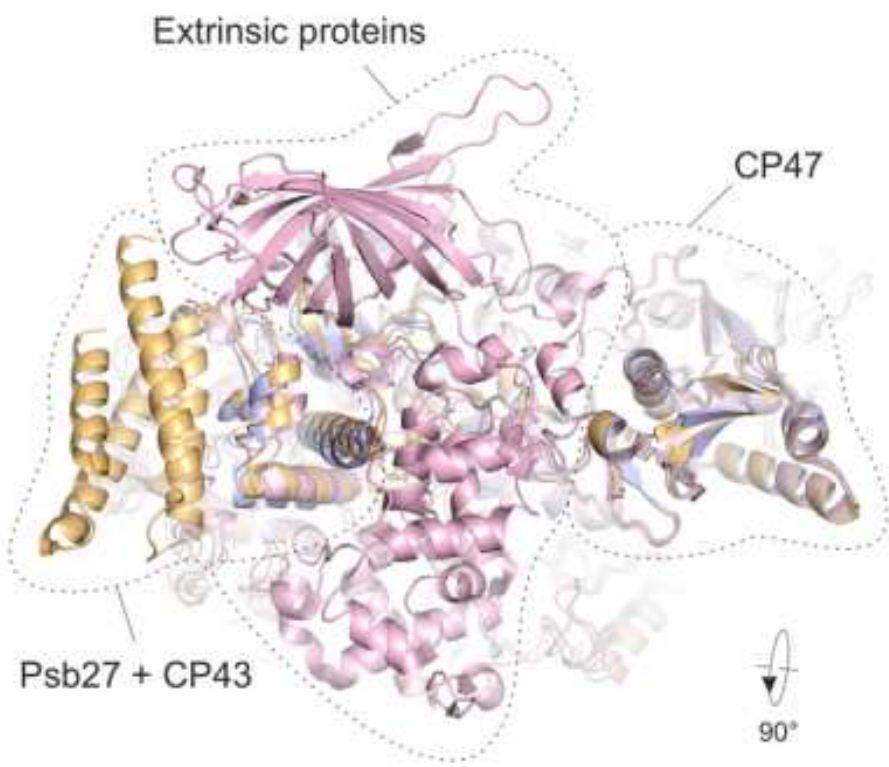

C

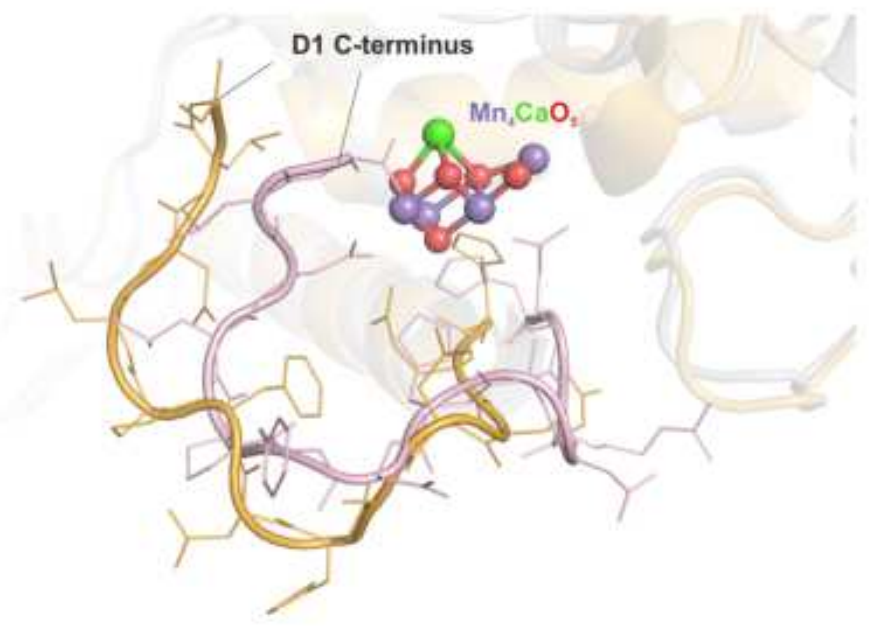

B

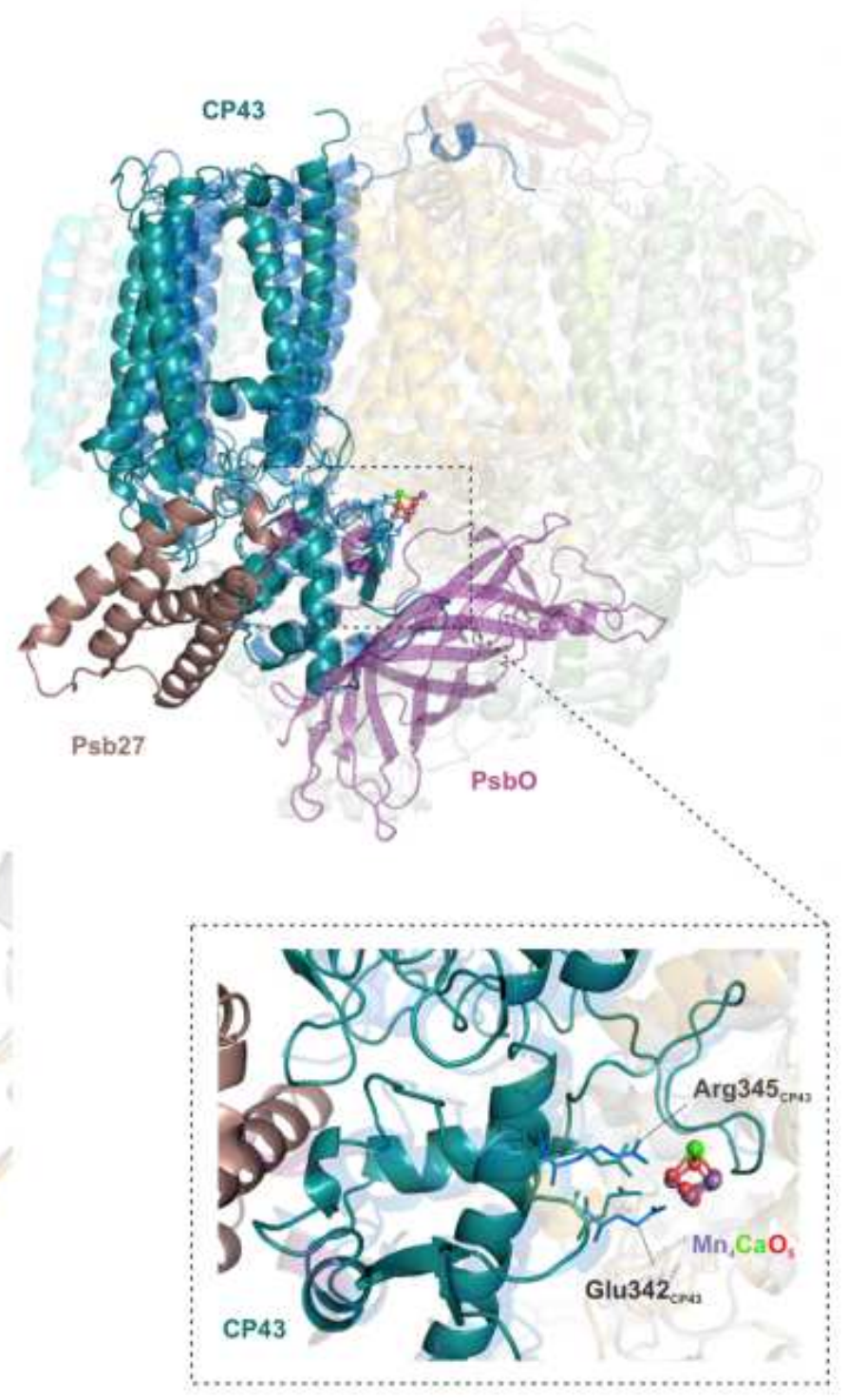


The role of Psb27 in Mn4CaO5 cluster assembly. (A) Bottom view of the luminal PSIl surface for PSIII (orange), the PSII-M control (light blue) and mature monomeric PSII (PDB-ID 3KZI) (light red). (B) Side view of CP43 (teal) and Psb27 (brown) in PSII-I, as well as of CP43 (blue) and PsbO (purple) in mature monomeric PSII (PDB-ID 3KZI). Dashed box: CP43 E loop with residues Arg345 and Glu342 (shown as sticks), which are involved in coordination of the $\mathrm{Mn} 4 \mathrm{CaO} 5$ cluster. We changed the numbering of $\mathrm{CP} 43$ residues due to a corrected $\mathrm{N}$-terminal sequence (www.UniProt.org). The residues correspond to Arg357 and Glu354 in previous publications. The high-resolution structure of the $\mathrm{Mn} 4 \mathrm{CaO} 5$ cluster is taken from Umena et al. 2011 (PDB-ID 3WU2). (C) Position of the D1 C-terminus in PSII-I (orange) and mature monomeric PSII (PDB-ID 3KZI) (light red). 

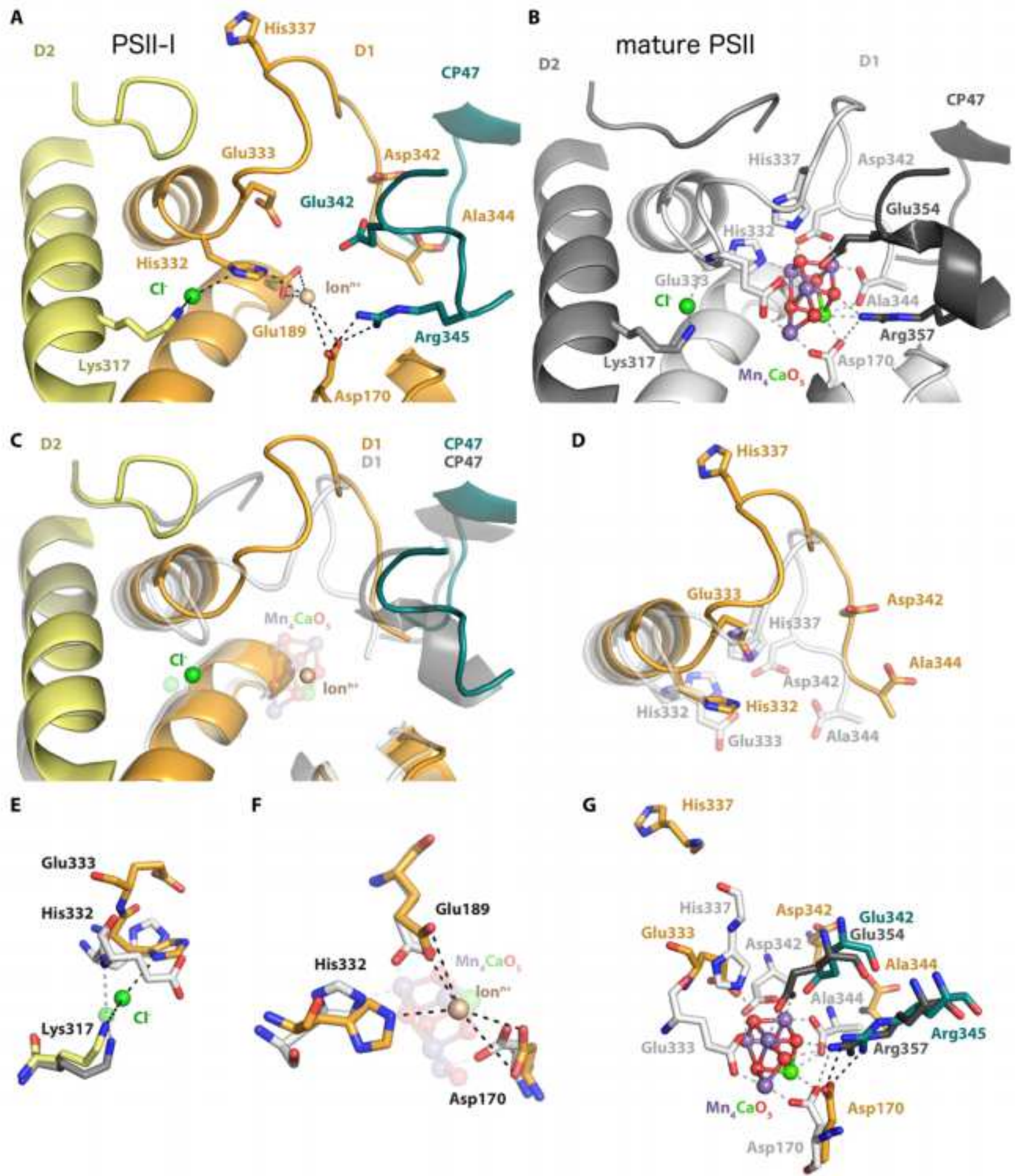

\section{Figure 7}

Conformational changes within the active site of the $\mathrm{Mn} 4 \mathrm{CaO} 5$ cluster. The Mn4CaO5 cluster performs PSII's unique water-splitting reaction. (A) The active site of the Mn4CaO5 cluster is resolved within our PSII-I structural model but is not yet oxygen-evolving. (B) Crystal structure of the oxygen-evolving, mature PSII (PDB-ID 3WU2, resolution 1.9 ̊̊). (C) Overlay of both structures, illustrating significant differences in the backbone conformation of the D1 and D2 C-terminal tails. (D) Accompanying side chain 
rearrangements of the $\mathrm{D} 1 \mathrm{C}$-terminus. The $\mathrm{Cl}-(\mathrm{E})$, lon+ $(\mathrm{F})$ and $\mathrm{Mn} 4 \mathrm{CaO} 5(\mathrm{G})$ cluster coordination partners are compared in detailed. The validation of the fit to density for the structural details shown here is provided in Figure S7.

\section{Supplementary Files}

This is a list of supplementary files associated with this preprint. Click to download.

- SupMovie1.mp4

- 03NaturePlantsSupplementary01 withref.pdf

- SupMovie2.mp4

- SupMovie3.mp4

- SupMovie4.mp4 